\title{
Curve complexes versus Tits buildings: structures and applications
}

\author{
Lizhen $J i^{*}$ \\ Department of Mathematics \\ University of Michigan \\ Ann Arbor, MI 48109 \\ (March 23, 2011)
}

\begin{abstract}
Tits buildings $\Delta_{\mathbb{Q}}(\mathbf{G})$ of linear algebraic groups $\mathbf{G}$ defined over the field of rational numbers $\mathbb{Q}$ have played an important role in understanding partial compactifications of symmetric spaces and compactifications of locally symmetric spaces, cohomological properties of arithmetic subgroups and S-arithmetic subgroups of $\mathbf{G}(\mathbb{Q})$. Curve complexes $\mathcal{C}\left(S_{g, n}\right)$ of surfaces $S_{g, n}$ were introduced to parametrize boundary components of partial compactifications of Teichmüller spaces and were later applied to understand properties of mapping class groups of surfaces and the geometry and topology of 3-dimensional manifolds. Tits buildings are spherical building. Another important class of buildings consists of Euclidean buildings, for example, the BruhatTits buildings of linear algebraic groups defined over local fields. In this chapter, we summarize and compare some properties and applications of buildings and curve complexes. We try to emphasize their similarities but also point out differences. In some sense, curve complexes are combinations of spherical, Euclidean and hyperbolic buildings. We hope that such a comparison might motivate more questions and at the same time suggest methods to solve them. Furthermore it might introduce buildings to people who study curve complexes and curve complexes to people who study buildings.
\end{abstract}

2000 Mathematics Subject Classification: 53C35, 30F60, 22E40, 20G15, 57M99.

Keywords: Tits building, curve complex, spherical building, Euclidean building, hyperbolic building, arithmetic group, mapping class group, symmetric space, Teichmüller space, proper action, classifying space, universal space, locally symmetric space, Mostow strong rigidity, moduli space, ending lamination conjecture, simplicial volume, compactification, boundary, duality group, cohomological dimension, asymptotic cone, Novikov conjecture, quasi-isometry, Heegaard splitting.

\footnotetext{
*Partially Supported by NSF grant DMS 0905283
} 


\section{Contents}

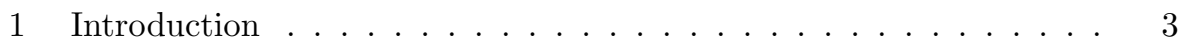

2 Definition of buildings . . . . . . . . . . . . 6

2.1 A geometric definition of Tits buildings via symmetric spaces . 6

2.2 Axioms for spherical buildings via apartments . . . . . . . 8

2.3 Euclidean and hyperbolic buildings . . . . . . . . . . . . 9

2.4 Rational Tits buildings of linear algebraic groups . . . . . . . 11

3 Definition of curve complexes . . . . . . . . . . . . . . . 12

4 Geometric and topological properties of buildings . . . . . . . . 14

5 Geometric and topological properties of curve complexes . . . . . 18

6 Selected applications of buildings . . . . . . . . . . . . . 21

6.1 Automorphism groups of buildings . . . . . . . . . . . 21

6.2 Mostow strong rigidity and generalizations . . . . . . . . . . 21

6.3 Compactifications of locally symmetric spaces . . . . . . . . 23

6.4 Cohomological dimension and duality properties of arithmetic

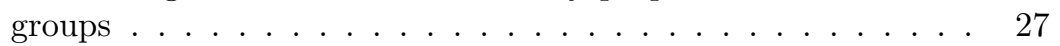

6.5 Simplicial volumes of locally symmetric spaces _ . . . . . . 29

6.6 Asymptotic cones of symmetric spaces and locally symmetric spaces . . . . . . . . . . . . . . . . 31

6.7 Applications of Euclidean buildings . . . . . . . . . . . . . 32

6.8 Compactifications of Euclidean buildings . . . . . . . . . . 34

7 Applications of curve complexes . . . . . . . . . . . . . . 37

7.1 Automorphism groups of curve complexes . . . . . . . . . 37

7.2 Isometry groups of Teichmüller spaces . . . . . . . . . . . 38

7.3 The ending lamination conjecture of Thurston . . . . . . . . . 39

7.4 Quasi-isometric rigidity of mapping class groups . . . . . . . 43

7.5 Finite asymptotic dimension of mapping class groups and the Novikov conjectures . . . . . . . . . . . . . . . . 44

7.6 Non-hyperbolicity of Weil-Petersson metric of Teichmüller space 48

7.7 Heegaard splittings and Hempel distance of 3-manifolds . . . . 49

7.8 Partial compactifications of Teichmüller spaces and their boundaries ........................ 50

7.9 Cohomological dimension and duality properties of mapping class groups . . . . . . . . . . . . . . . . 53

7.10 Tangent cones at infinity of Teichmüller spaces, moduli spaces, and mapping class groups . . . . . . . . . . . . . . . . 54

7.11 Simplicial volumes of moduli spaces $\ldots \ldots \ldots \ldots 55$

7.12 The action of $\operatorname{Mod}_{g, n}$ on $\mathcal{C}\left(S_{g, n}\right)$ and applications . . . . 57 


\section{Introduction}

\section{Summary.}

Tits buildings $\Delta_{\mathbb{Q}}(\mathbf{G})$ of linear algebraic groups $\mathbf{G}$ defined over $\mathbb{Q}$ have played an important role in understanding partial compactifications of symmetric spaces and compactifications of locally symmetric spaces, cohomological properties of arithmetic subgroups and S-arithmetic subgroups of $\mathbf{G}(\mathbb{Q})$. On the other hand, curve complexes $\mathcal{C}\left(S_{g, n}\right)$ of surfaces $S_{g, n}$ were introduced to parametrize boundary components of partial compactifications of Teichmüller spaces and were later applied to understand properties of mapping class groups of surfaces and the geometry and topology of 3-dimensional manifolds.

Tits buildings are spherical buildings. Another important class of buildings consists of Euclidean buildings, for example, the Bruhat-Tits buildings of linear algebraic groups defined over local fields. In this chapter, we summarize and compare some properties and applications of buildings and curve complexes. We try to emphasize their similarities but also point out differences. In some sense, curve complexes are combinations of spherical, Euclidean and hyperbolic buildings. We hope that such a comparison might motivate more questions and at the same time suggest methods to solve them. Furthermore it might introduce buildings to people who study curve complexes and curve complexes to people who study buildings.

The origin of Tits buildings.

Buildings were originally introduced by Tits [166] [167] in order to realize exceptional Lie groups as the symmetry groups of spaces (or geometry) ${ }^{1}$ so that one can construct geometrically analogues of exceptional simple Lie groups over arbitrary fields. ${ }^{2}$ See [152] for an overview of motivations and the history of Tits buildings.

Since then, Tits buildings have been applied to many different situations with great success. There are several types of buildings: (1) spherical buildings, for example, Tits buildings, (2) Euclidean buildings, for example, Bruhat-Tits buildings, (3) hyperbolic buildings, (4) $\mathbb{R}$-buildings, (5) topological buildings, (6) twin buildings. We will recall briefly some of these buildings below. See the book [2] for detailed definitions and structures of buildings, and the survey [87] for references on many different applications of buildings.

\footnotetext{
${ }^{1}$ Classical simple Lie groups over $\mathbb{C}$ are the symmetry groups of quadratic forms or sesquilinear forms of finite dimensional vector spaces over $\mathbb{C}$. The same construction works for vector spaces over finite fields and produces classical finite groups of Lie type, but this method does not extend to exceptional simple Lie groups.

${ }^{2}$ According to $[153$, p. 292], “... it is perhaps worth remarking that one of the initial motivations for the theory of buildings, at a time when Chevalley's fundamental "Tohoku paper" had not yet appeared, was the search for a geometric way of obtaining algebraic analogues of the exceptional Lie groups."
} 
There are several basic ways in which Tits buildings are used:

(1) Buildings describe the large-scale geometry or the geometry at infinity of Lie groups, symmetric spaces and locally symmetric spaces.

(2) Buildings describe the topology at infinity of partial compactifications of symmetric spaces and of the boundary of compactifications of locally symmetric spaces, and also the topology of the ends of locally symmetric spaces.

(3) Buildings provide natural combinatorial and metric spaces on which groups such as Lie groups, arithmetic subgroups and $p$-adic Lie groups act.

(4) Buildings can be used to study cohomological properties of arithmetic groups.

We are mainly interested in infinite buildings in this chapter. For finite buildings and their applications in finite groups and their representation theory, see [44].

The origin of curve complexes.

Motivated by the Borel-Serre compactification of locally symmetric spaces [20], Harvey [69] [70] [73] introduced the curve complex $\mathcal{C}\left(S_{g}\right)$ of a compact oriented surface $S_{g}$ to parametrize the boundary components of partial compactifications of the Teichmüller space $\mathcal{T}_{g}$, the space of marked compact Riemann surfaces of genus $g$ which induce compactifications of the moduli space $\mathcal{M}_{g}$ of Riemann surfaces of genus $g$. In some sense, it was an exact analogue of the spherical Tits building $\Delta_{\mathbb{Q}}(\mathbf{G})$ of a linear semisimple algebraic group G defined over $\mathbb{Q}$ which serves as a parameter space for the boundary components of the Borel-Serre partial compactification of the symmetric space $X=G / K$. The same definition works for a more general oriented surface $S_{g, n}$ of genus $g$ with $n$ punctures and gives a curve complex $\mathcal{C}\left(S_{g, n}\right)$. Motivated by the analogy between arithmetic groups and mapping class groups $\operatorname{Mod}_{g, n}=\operatorname{Diff}^{+}\left(S_{g, n}\right) / \operatorname{Diff}^{0}\left(S_{g, n}\right)$, the curve complex $\mathcal{C}\left(S_{g, n}\right)$ was used to study cohomological properties of $\operatorname{Mod}_{g, n}$ [66] [80] [69].

It turns out that the curve complexes $\mathcal{C}\left(S_{g, n}\right)$ can also be used to study many problems in (lower dimensional) topology and geometry, in particular the ending lamination conjecture of Thurston for 3-dimensional hyperbolic manifolds, quasi-isometric rigidity of mapping class groups $\operatorname{Mod}_{g, n}$ and the finiteness of the asymptotic dimension of $\operatorname{Mod}_{g, n}$.

Some applications of curve complexes are motivated by results for Tits buildings, and others are quite different. There are several basic ways in which the curve complexes are used.

(1) Curve complexes describe the large scale geometry or the geometry at infinity of Teichmüller spaces and of the moduli spaces of Riemann surfaces. 
(2) Curve complexes parametrize boundary components of partial compactifications of Teichmüller spaces, they describe the topology at infinity of these partial compactifications, and they also describe the boundary of compactifications of moduli spaces, and the topology of the ends of moduli spaces.

(3) Curve complexes provide natural combinatorial and metric spaces on which mapping class groups act, and structures and representations of the mapping class groups can be understood and constructed from these actions.

(4) Curve complexes can be used to study cohomological properties of mapping class groups.

(5) Surfaces and simple closed curves occur naturally in the study of 3dimensional manifolds and hence curve complexes can be used to understand 3-dimensional geometry and topology.

In this chapter, we will present some applications of buildings and curve complexes to support these general points. The above two lists suggest many similarities between these two classes of simplicial complexes. On the other hand, there are also dramatic differences between buildings and curve complexes.

Besides the curve complex $\mathcal{C}\left(S_{g, n}\right)$, there are also several related complexes for surfaces $S_{g, n}$, which are important to understand the geometry and topology of Teichmüller spaces $\mathcal{T}_{g, n}$ and mapping class groups $\operatorname{Mod}_{g, n}$. We will address these issues as well.

This chapter can be seen as a sequel of the survey papers [87] and [88] in some sense. Since it emphasizes curve complexes and their applications, it can complement the other two papers.

Acknowledgments. This chapter is an expanded version of lecture notes of an invited talk at International Conference on Buildings, Finite geometries and Groups in Bangalore, India, August 29-31, 2010. I would like to thank the organizers for their invitation. This chapter was written for the book with the same title in the series "Springer Proceedings in Mathematics". I would like to thank Springer and the editor of the book, N. Sastry, for their permission to reprint this chapter in the Handbook of Teichmüller theory.

I would also like to thank Dick Canary for many helpful suggestions and references, Bill Harvey for sending valuable reprints, Feng Luo, Juan Souto and Ralf Spatzier for helpful correspondences, Misha Kapovich and B. Sury for helpful comments, and Athanase Papadopoulos for reading several versions of the chapter very carefully and for many helpful comments. I would also like to thank an anonymous referee for helpful suggestions and comments. A part of the work in this chapter was done during a visit to MSC, Tshinghua 
University, Beijing, in 2010 and I would like to thank the people at this center for providing a stimulating environment.

\section{Definition of buildings}

In this section, we first introduce in $\S 2.1$ the spherical Tits building $\Delta(G)$ of a semisimple Lie group $G$ through a classification of geodesics in the associated symmetric space $X=G / K$. This justifies the point of view that $\Delta(G)$ describes the geometry at infinity of the symmetric space $X$. Then in $\S 2.2$, we discuss a more common definition of buildings through a system of apartments. In $\S 2.3$, we introduce Euclidean and hyperbolic buildings and we mention other buildings. In $\S 2.4$, we define the rational Tits building $\Delta_{\mathbb{Q}}(\mathbf{G})$ of a linear algebraic group $\mathbf{G}$ defined over $\mathbb{Q}$.

\subsection{A geometric definition of Tits buildings via symmetric spaces}

Let $G$ be a semisimple noncompact Lie group with finitely many connected components, and let $K \subset G$ be a maximal compact subgroup. Then $X=G / K$ with an invariant metric is a symmetric space of noncompact type, and $X$ is a simply connected nonpositively curved Riemannian manifold. Let $X(\infty)$ be the set of equivalence classes of geodesics in $X$. Specifically, we assume that all geodesics in $X$ are of unit speed and directed. Two geodesics $\gamma_{1}(t)$ and $\gamma_{2}(t)$ in $X$ are defined to be equivalent if

$$
\limsup _{t \rightarrow+\infty} d\left(\gamma_{1}(t), \gamma_{2}(t)\right)<+\infty,
$$

where $d(\cdot, \cdot)$ is the distance function of $X$. It can be shown that for any point $x_{0} \in X, X(\infty)$ can be canonically identified with the unit sphere in the tangent space $T_{x_{0}} X$. It is known that there is a natural topology on $X \cup X(\infty)$ such that it is a compactification of $X$, called the geodesic compactification, or visual compactification, and $X(\infty)$ is hence called the sphere at infinity (or visual sphere) of $X$. See [4].

When $X=\mathrm{SL}(2, \mathbb{R}) / \mathrm{SO}(2)$ is identified with the upper half place $\mathbf{H}^{2}$, then $X(\infty)=\mathbb{R} \cup\{i \infty\}$. When $X=\mathrm{SL}(2, \mathbb{R}) / \mathrm{SO}(2)$ is identified with the unit disc $D=\{z \in \mathbb{C}|| z \mid<1\}$, then $X(\infty)$ is equal to the unit circle $S^{1}=\partial D$.

Clearly the isometry action of $G$ on $X$ preserves the equivalence relation between geodesics and hence acts on $X(\infty)$.

A natural question is whether these points in $X(\infty)$, i.e, equivalence classes of geodesics, are the same, i.e., belong to one common $G$-orbit. If the answer is negative, a natural problem is to parametrize $G$-orbits in $X(\infty)$. 
It turns out that both questions can be answered using parabolic subgroups $P$ of $G$, i.e., closed subgroups $P$ such that $G / P$ is compact. If $P=G$, it is a parabolic subgroup by definition. Any parabolic subgroup $P$ with $P \neq G$ is called a proper parabolic subgroup of $G$. The set of proper parabolic subgroups of $G$ is a partially ordered set by inclusion, and there are infinitely many maximal and minimal elements in this partially ordered set, which are called maximal proper parabolic subgroups and minimal parabolic subgroups of $G$.

Proposition 2.1. The $G$-action on $X$ extends to a continuous action on $X \cup X(\infty)$. For any point $z \in X(\infty)$, its stabilizer $G_{z}$ in $G$ is a proper parabolic subgroup, and every proper parabolic subgroup $P$ of $G$ arises as the stabilizer of a point $z \in X(\infty)$.

For each proper parabolic subgroup $P$ of $G$, let $\sigma_{P}$ be the set of points in $X(\infty)$ that are fixed by $P$, equivalently the set of points $z \in X(\infty)$ whose stabilizer $G_{z}$ in $G$ contains $P$.

Proposition 2.2. For every proper parabolic subgroup $P, \sigma_{P}$ is a spherical simplex, and its interior $\sigma_{P}^{o}$, i.e., the open simplex when $\sigma_{P}$ is considered as a simplex, is equal to the set of points $z$ whose stabilizer is equal to $P$. The simplex $\sigma_{P}$ consists of one point if and only if $P$ is a maximal proper parabolic subgroup. If $P$ is a minimal parabolic subgroup of $G$, then the dimension of $\sigma_{P}$ is equal to $r-1$, where $r$ is the rank of $X$, i.e., the maximal dimension of totally geodesic flat submanifolds of $X$. The decomposition of $X(\infty)$ into $\sigma_{P}$,

$$
X(\infty)=\cup_{P} \sigma_{p}=\coprod_{P} \sigma_{P}^{0},
$$

gives $X(\infty)$ the structure of an infinite simplicial complex, denoted by $\Delta(X)$.

The simplicial complex $\Delta(X)$ is called the Tits building associated with the symmetric space $X$ of noncompact type. If the rank $r$ of $X$ is equal to 1 , then $\Delta(X)$ is a 0 -simplicial complex, i.e., a disjoint union of points. If $r>0$, it can be shown that $\Delta(X)$ is connected. This can be seen from the axioms of buildings in the next subsection.

By definition, $\Delta(X)$ classifies geodesics of $X$ into different types.

Proposition 2.3. Let $P_{0}$ be a minimal parabolic subgroup of $G$. Then every $G$-orbit in $X(\infty)$ meets $\sigma_{P_{0}}$ in exactly one point. $G$ acts on the Tits building $\Delta(X)$ by simplicial maps, and every simplex $\sigma_{P}$ is contained in the $G$-orbit of a face of $\sigma_{P_{0}}$, i.e., $G \backslash \Delta(X)$ can be identified with $\sigma_{P_{0}}$.

Corollary 2.4. $G$ acts transitively on $X(\infty)$ if and only if a minimal parabolic subgroup of $G$ is also a maximal parabolic subgroup $G$, i.e., the rank $r$ of $X$ is equal to 1 . 
For more discussion and proofs of the above results, see [60].

\subsection{Axioms for spherical buildings via apartments}

The Tits building $\Delta(X)$ defined in the previous subsection can be described directly in terms of proper parabolic subgroups of $G$.

Let $\mathcal{P}$ be the partially ordered set of proper parabolic subgroups of $G$, where the partial order is given by containment, i.e., the opposite of the inclusion mentioned in the previous subsection. The structure theory of parabolic subgroups of $G$ shows that this poset can be realized by an infinite simplicial complex $\Delta(G)$ satisfying the following conditions:

(1) Every parabolic subgroup $P \in \mathcal{P}$ corresponds to a unique simplex $\sigma_{P}$ in $\Delta(G)$, and every simplex of $\Delta(G)$ is of this form.

(2) For any two parabolic subgroups $P_{1}, P_{2} \in \mathcal{P}, P_{1} \subset P_{2}$ if and only if $\sigma_{P_{1}}$ contains $\sigma_{P_{2}}$ as a face.

(3) A simplex $\sigma_{P}$ is a point if and only if $P$ is a maximal parabolic subgroup of $G$. For a non-maximal parabolic subgroup $P$, the vertices of $\sigma_{P}$ correspond to maximal parabolic subgroups that contain $P$.

This simplicial complex $\Delta(G)$ is called the Tits building of $G$. By Proposition 2.2, $\Delta(G)$ is isomorphic to $\Delta(X)$. These are important examples of spherical Tits buildings.

Definition 2.5. A simplicial complex $\Delta$ is called a spherical Tits building if it contains a family of subsets called apartments and satisfies the following conditions:

(1) Every apartment is a finite Coxeter complex.

(2) Any two simplices are contained in some apartment.

(3) Given two apartments $\Sigma$ and $\Sigma^{\prime}$ and simplices $\sigma, \sigma^{\prime} \in \Sigma \cap \Sigma^{\prime}$, there exists an isomorphism of $\Sigma$ onto $\Sigma^{\prime}$ which keeps $\sigma, \sigma^{\prime}$ pointwise fixed.

In the above definition, for any finite Coxeter group $W$, i.e., a finite group generated by reflections with respect to hyperplanes in a fixed Euclidean space, there is a Coxeter complex, which is a finite simplicial complex constructed as follows. Every reflection $\alpha \in W$ fixes a hyperplane $\mathcal{H}_{\alpha}$. The collection of such hyperplanes $\mathcal{H}_{\alpha}$ is invariant under $W$. Connected components of the complement of the union of $\left\{\mathcal{H}_{\alpha}\right\}$ in $V$ are called chambers, which are simplicial cones. The chambers and their faces together give a partition of $V$ into simplicial cones. Let $S$ be the unit sphere in $V$. Then the intersection of $S$ with these simplicial cones gives a finite simplicial complex, called the Coxeter complex of $W$, whose underlying topological space is $S$, i.e., the Coxeter complex gives a finite triangulation of the unit sphere. 
To see that $\Delta(X)$ is a spherical Tits buildings, we start with the construction of apartments. For any flat totally geodesic submanifold $F$ of $X$, which is isometric to $\mathbb{R}^{r}$, its closure in $X \cup X(\infty)$ is equal to $F \cup F(\infty)$, where $F(\infty)$ is homeomorphic to the sphere $S^{r-1}$. The simplicial complex structure of $X(\infty)$ given by $\Delta(X)$ induces a simplicial complex structure on $F(\infty)$. In fact, $F(\infty)$ is equal to the union of $\sigma_{P}$ for some parabolic subgroups $P$. Denote this finite simplicial complex by $\Sigma_{F}$. Then it is a Coxeter complex associated with the Weyl group of the Lie group $G$.

Proposition 2.6. With respect to the collection of finite subcomplexes $\Sigma_{F}$ associated with flat subspaces $F$, the infinite simplicial complex $\Delta(X)$ is a spherical Tits building.

To understand the spherical building structure of $\Delta(G)$, we need to define apartments in terms of group structure. For any maximal compact subgroup $K$ of $G$, let $\mathfrak{g}=\mathfrak{k} \oplus \mathfrak{p}$ be the Cartan decomposition. Let $\mathfrak{a} \subset \mathfrak{p}$ be a maximal abelian subalgebra. Let $A=\exp \mathfrak{a}$ be the corresponding Lie subgroup of $G$. Then there are only finitely many parabolic subgroups $P$ that contain $A$, and the union of their simplices $\sigma_{P}$ gives a triangulation of the unit sphere $\mathfrak{a}(+\infty)$ of $\mathfrak{a}$ and is a Coxeter complex for the Weyl group of $G$. Denote it by $\Sigma_{\mathfrak{a}}$.

Proposition 2.7. With respect to the collection of finite subcomplexes $\Sigma_{\mathfrak{a}}$ associated with maximal abelian subspaces, the infinite simplicial complex $\Delta(X)$ is a spherical Tits building.

Proposition 2.7 is proved using the Bruhat decomposition. To derive Proposition 2.6 from Proposition 2.7, we need to identify their apartments.

The finite simplicial complexes $\Sigma_{F}$ and $\Sigma_{\mathfrak{a}}$ can be identified as follows. For any maximal flat totally geodesic submanifold $F$ of $X$, let $x_{0} \in F$ be any point, and $K=G_{x_{0}}$ be the stabilizer of $x_{0}$ in $G$. Then there exists a maximal abelian subalgebra $\mathfrak{a} \subset \mathfrak{p}$ with respect to the Cartan decomposition of $\mathfrak{g}$ induced by $K$ such that $F=\exp \mathfrak{a} x_{0}$, and $\Sigma_{F}$ is identified with $\Sigma_{\mathfrak{a}}$ under the identification between $\Delta(X)$ and $\Delta(G)$. For the proof of the above propositions and more discussion, see the book [60] and references therein.

\subsection{Euclidean and hyperbolic buildings}

It will be shown below that the curve complex $\mathcal{C}\left(S_{g, n}\right)$ shares some properties with spherical buildings, and also with Euclidean buildings and hyperbolic buildings. For convenience, we briefly introduce their definitions here.

First we define Euclidean reflection groups. Let $V$ be a Euclidean space. An affine reflection group $W$ on $V$ is a group of affine isometries generated

by reflections with respect to affine hyperplanes such that the set $\mathcal{H}$ of affine 
hyperplanes fixed by reflections in $W$ is locally finite. Clearly, a finite reflection group is an affine reflection group. Assume that $W$ is an infinite irreducible reflection group. Then the hyperplanes in $\mathcal{H}$ divide $V$ into simplices, and $W$ acts simply transitively on the set of simplices. These simplices and their faces form a Euclidean Coxeter complex.

Definition 2.8. A simplicial complex $\Delta$ is called a Euclidean building if it contains a family of subsets called apartments and satisfies the following conditions:

(1) Every apartment is an infinite Euclidean Coxeter complex.

(2) Any two simplices are contained in some apartment.

(3) Given two apartments $\Sigma$ and $\Sigma^{\prime}$ and simplices $\sigma, \sigma^{\prime} \in \Sigma \cap \Sigma^{\prime}$, there exists an isomorphism from $\Sigma$ onto $\Sigma^{\prime}$ which keeps $\sigma, \sigma^{\prime}$ pointwise fixed.

An important source of Euclidean buildings comes from the Bruhat-Tits building $\Delta^{B T}(\mathbf{G})$ associated with a linear semisimple algebraic group $\mathbf{G}$ defined over a non-Archimedean local field $k$. In this case, the simplices of $\Delta^{B T}(\mathbf{G})$ are parametrized by parahoric subgroups of $\mathbf{G}(k)$. There is no simple definition or characterization of parahoric subgroups of $\mathbf{G}(k)$ as in the case of parabolic subgroups. When $\mathbf{G}$ is simply connected, any maximal open compact subgroup of $\mathbf{G}(k)$ is a maximal parahoric subgroup, and the converse is also true. See [87, §3] for more details and references.

Now we introduce hyperbolic buildings. Let $\mathbf{H}^{n}$ be the real hyperbolic space of dimension $n$. Let $P$ be a compact convex hyperbolic polyhedron such that the reflections with respect to its codimension 1 faces generate a group $W$ that acts properly on $\mathbf{H}^{n}$ with $P$ as a fundamental domain. Then the totally geodesic hypersurfaces of $\mathbf{H}^{n}$ that are fixed by reflections in $W$ are locally finite, and the connected components of the complement of the union of these hyperplanes are hyperbolic polyhedra. These polyhedra and their faces form a polyhedral complex, which is called a hyperbolic Coxeter complex associated with $P$. Hyperbolic Coxeter complexes can only exist for $n \leq 29$.

Definition 2.9. A polyhedral complex $\Delta$ is called a hyperbolic building of type $P$ if it contains a family of subcomplexes called apartments and satisfies the following conditions:

(1) Every apartment is a hyperbolic Coxeter complex determined by $P$.

(2) Any two polyhedra are contained in some apartment.

(3) Given two apartments $\Sigma$ and $\Sigma^{\prime}$ and polyhedra $\sigma, \sigma^{\prime} \in \Sigma \cap \Sigma^{\prime}$, there exists an isomorphism of $\Sigma$ onto $\Sigma^{\prime}$ which keeps $\sigma, \sigma^{\prime}$ pointwise fixed.

See [55] for more detail and examples. When $n=2, P$ is given by a compact polygon in the hyperbolic plane $\mathbf{H}^{2}$ such that the angle at each vertex is equal 
to $\pi / m$ for some integer $m$, and we get a hyperbolic building of Fuchsian type $[22]$.

A natural generalization of spherical buildings is the notion of twin buildings. An important example is a pair of spherical Tits buildings associated to one linear algebraic group, for example, $\mathrm{SL}\left(n, F_{p}\left[t, t^{-1}\right]\right)$. See [1] for the definition and applications to $S$-arithmetic subgroups of linear algebraic groups defined over function fields. See [45] [46] for a more geometric group theoretic point of view of buildings and Coxeter complexes.

\subsection{Rational Tits buildings of linear algebraic groups}

As explained before, for any semisimple Lie group $G$, there is a spherical Tits building $\Delta(G)$ that can be related to the geodesic compactification of its symmetric space $X=G / K$. If $G$ is the real locus of a linear algebraic group $\mathbf{G} \subset G L(n, \mathbb{C})$ defined over $\mathbb{Q}$ with positive $\mathbb{Q}$-rank, then there are several other spherical Tits buildings associated with $\mathbf{G}$. They are important for compactifications of locally symmetric spaces and in the study of arithmetic subgroups of $\mathbf{G}(\mathbb{Q})$ and more generally $S$-arithmetic subgroups of $\mathbf{G}(\mathbb{Q})$.

Let $\mathcal{P}_{\mathbb{Q}}$ be the set of $\mathbb{Q}$-parabolic subgroups of $\mathbf{G}$, i.e., parabolic subgroups defined over $\mathbb{Q}$. Then there is an infinite simplicial complex $\Delta_{\mathbb{Q}}(\mathbf{G})$ whose simplices $\sigma_{\mathbf{P}}$ are parametrized by parabolic subgroups $\mathbf{P}$ in $\mathcal{P}_{\mathbb{Q}}$ and which satisfies the following conditions:

(1) When $\mathbf{P}$ is maximal $\mathbb{Q}$-parabolic subgroup, $\sigma_{\mathbf{P}}$ is a point.

(2) For two $\mathbb{Q}$-parabolic subgroups $\mathbf{P}_{1}, \mathbf{P}_{2}$, the inclusion relation $\mathbf{P}_{1} \subset \mathbf{P}_{2}$ holds if and only if $\sigma_{\mathbf{P}_{1}}$ contains $\sigma_{\mathbf{P}_{2}}$ as a face.

The simplicial complex $\Delta_{\mathbb{Q}}(\mathbf{G})$ is called the $\mathbb{Q}$-Tits building of $\mathbf{G}$. It cannot be realized as the boundary of a compactification of $X$ as the Tits building $\Delta(G)$, where $G=\mathbf{G}(\mathbb{R})$ is the real locus of $\mathbf{G}$, or as a subset of the boundary of a compactification of $X$ if the $\mathbb{R}$-rank of $\mathbf{G}$ is strictly greater than the $\mathbb{Q}$-rank of $\mathbf{G}$. But $\Delta_{\mathbb{Q}}(\mathbf{G})$ can be realized as the boundary of a partial compactification of $X$, which gives the Tits compactification of $\Gamma \backslash X$ in [94].

The main application of $\Delta_{\mathbb{Q}}(\mathbf{G})$ is that $\Delta_{\mathbb{Q}}(\mathbf{G})$ naturally parametrizes boundary components of partial compactifications of $X$ whose quotients by arithmetic subgroups $\Gamma \subset \mathbf{G}(\mathbb{Q})$ are compactifications of locally symmetric spaces $\Gamma \backslash X$. See $\S 6.3$.

For every prime $p$, let $\mathbb{Q}_{p}$ be the field of $p$-adic numbers. When $\mathbf{G}$ is considered as a linear algebraic group defined over $\mathbb{Q}_{p}$, the set of parabolic subgroups of $\mathbf{G}$ defined over $\mathbb{Q}_{k}$ gives a spherical Tits building $\Delta_{\mathbb{Q}_{p}}(\mathbf{G})$.

As mentioned before, there is a Euclidean building, the Bruhat-Tits building, associated with the $p$-adic Lie group $\mathbf{G}\left(\mathbb{Q}_{p}\right)$. Denote this building by $\Delta^{B T}\left(\mathbf{G}\left(\mathbb{Q}_{p}\right)\right)$. We will see below that $\Delta^{B T}\left(\mathbf{G}\left(\mathbb{Q}_{p}\right)\right)$ can be compactified by adding the spherical building $\Delta_{\mathbb{Q}_{p}}(\mathbf{G})$ (Proposition 6.25). 


\section{Definition of curve complexes}

Let $S_{g, n}$ be an oriented surface of genus $g$ with $n$ punctures (or $n$ boundary components). In the rest of this paper, we assume that the Euler characteristic $\chi\left(S_{g, n}\right)$ is negative so that $S_{g, n}$ admits complete hyperbolic metrics of finite area.

A simple closed curve $c$ in $S_{g, n}$ is called essential if it is not homotopic to a point or a loop around a puncture or a boundary component.

The curve complex $\mathcal{C}\left(S_{g, n}\right)$ is a simplicial complex such that:

(1) Its vertices are parametrized by homotopy classes of essential simple closed curves $[c]$ in $S_{g, n}$.

(2) Homotopy classes $\left[c_{0}\right],\left[c_{1}\right], \cdots,\left[c_{k}\right]$ form the vertices of a $k$-simplex if and only if they are pairwise distinct and admit disjoint representatives.

If we put a complete hyperbolic metric of finite area and with geodesic boundary on $S_{g, n}$, then the homotopy class of each essential simple closed curves in $S_{g, n}$ contains a unique simple closed geodesic, and hence the vertices of $\mathcal{C}\left(S_{g, n}\right)$ corresponds to simple closed geodesics of the hyperbolic metric.

The simplest example is $\mathcal{C}\left(S_{1,1}\right)$. Let $\Sigma_{1,1}$ be a hyperbolic surface of genus 1 with one puncture. In this case, for every simple closed geodesic of $\Sigma_{1,1}$, there is no other simple closed geodesic that is disjoint from it. Therefore, $\mathcal{C}\left(S_{1,1}\right)$ is a countable collection of points and can be identified with the rational boundary points $\mathbb{Q} \cup\{\infty\}$ of the upper half-plane $\mathbf{H}^{2}$. For the surface $S_{1,1}$, a slight modification gives an interesting complex $\mathcal{C}_{1}\left(S_{1,1}\right)$. The vertices of $\mathcal{C}_{1}\left(S_{1,1}\right)$ still correspond to simple closed geodesics $\gamma$ in $\Sigma_{1,1}$, and $k+1$ simple closed geodesics $\gamma_{1}, \cdots, \gamma_{k+1}$ form the vertices of a $k$-simplex if and only if for every pair of distinct geodesics $\gamma_{i}$ and $\gamma_{j}$, the intersection number $\iota\left(\gamma_{i}, \gamma_{j}\right)= \pm 1$. Note that $\mathcal{C}_{1}\left(S_{1,1}\right)$ is 2 -dimensional. Then the 1 -skeleton of $\mathcal{C}_{1}\left(S_{1,1}\right)$ can be identified with the Farey graph, whose vertices are numbers in $\mathbb{Q} \cup\{\infty\}$, the vertex $\infty$ is joined to every integer $n$, and two points $\frac{r}{s}$ and $\frac{x}{y}$ in reduced form (i.e., the numerators and denominators do not contain common primes) are connected by an edge if and only if $r y-s x= \pm 1$. The simplicial complex $\mathcal{C}_{1}\left(S_{1,1}\right)$ gives an ideal triangulation (or tessellation) of the upper half plane $\mathbf{H}^{2}$, which is equal to the Farey tessellation, and hence $\mathcal{C}_{1}\left(S_{1,1}\right)$ is contractible. (It should be emphasized that a general curve complex $\mathcal{C}_{1}\left(S_{g, n}\right)$ is not contractible, and its nontrivial homotopy is used crucially to prove that the mapping class group $\operatorname{Mod}_{g, n}$ is not a virtual Poincaré duality group. See $\S 7.9$ below.)

In the general case, $\mathcal{C}\left(S_{g, n}\right)$ is fairly complicated and there is no simple geometric model. For example, it is not locally finite in general. It is not locally finite because the complement of a simple closed curve on a surface contains infinitely many homotopy classes of essential simple closed curves except in some special cases (the torus with at most one hole or the sphere with at most four holes). Note that $\mathcal{C}\left(S_{1,1}\right)$ is a disjoint union of countably many 
points and hence is locally finite. But the non-locally finiteness phenomenon can be seen in the above example $\mathcal{C}_{1}\left(S_{1,1}\right)$ already. One way to see this is to note that $\operatorname{SL}(2, \mathbb{Z})$ acts on the upper half plane, the Farey tessellation and the Farey graph. Each vertex of $\mathcal{C}_{1}\left(S_{1,1}\right)$ has an infinite stabilizer in $\operatorname{SL}(2, \mathbb{Z})$, which permutes the 2-simplices that contain the vertex, and hence there are infinitely many edges of $\mathcal{C}_{1}\left(S_{1,1}\right)$ out of each vertex, and $\mathcal{C}_{1}\left(S_{1,1}\right)$ is not locally finite.

The curve complex is not "homogeneous" in general as in the case of buildings. We will discuss some of its properties later.

Compared with the definition of the spherical Tits building $\Delta(G)$, this suggests that a homotopy class $[c]$ of essential simple closed curves plays a role similar to the one of a maximal parabolic subgroup, and a simplex of $\mathcal{C}\left(S_{g, n}\right)$ plays the role of a parabolic subgroup. More specifically, we have the following similarities:

(1) The boundary components of the Borel-Serre partial compactification of a symmetric space $X$ are parametrized by proper $\mathbb{Q}$-parabolic subgroups of $\mathbf{G}$, and maximal boundary components correspond to maximal $\mathbb{Q}$ parabolic subgroups. On the other hand, the boundary components of an analogous Borel-Serre partial compactification of the Teichmüller space $\mathcal{T}_{g, n}$ or of the completion of $\mathcal{T}_{g, n}$ in the Weil-Petersson metric are parametrized by simplices of $\mathcal{C}\left(S_{g, n}\right)$, and the maximal boundary components correspond to the vertices. (See $\S 6$ and $\S 7$ below for more details).

(2) For every $\mathbb{Q}$-parabolic subgroup $\mathbf{P}$ of $\mathbf{G}$, there is an associated Langlands decomposition of $X$. When $\mathbf{P}$ is a minimal $\mathbb{Q}$-parabolic subgroup, it is reduced to the Iwasawa decomposition of $X$ (or rather of the Lie group $G)$. On the other hand, for every top-dimensional simplex of $\mathcal{C}\left(S_{g, n}\right)$, there is a Fenchel-Nielsen coordinate system (or decomposition) of $\mathcal{T}_{g, n}$.

It is easy to see that the maximal number of homotopy classes of essential simple closed curves with disjoint representatives is equal to $3 g-3+n$. Therefore $\mathcal{C}\left(S_{g, n}\right)$ has dimension $3 g-4+n$. Simplices of $\mathcal{C}\left(S_{g, n}\right)$ with dimension equal to $3 g-4+n$ play a similar role as minimal parabolic subgroups.

There are also several other related complexes.

(1) The arc complex for surfaces with at least one puncture or boundary component in [66] [67] [106]. The topology of the arc complex at infinity is closely related to the topology of the curve complex $\mathcal{C}\left(S_{g, n}\right)[67$, Theorem 3.4]. The arc complex is used crucially in obtaining a $\operatorname{Mod}_{g, n^{-}}$ equivariant cell decomposition and a spine of optimal dimension of $\mathcal{T}_{g, n}$ when $n>0$ [67, Theorem 1.3 and Theorem 2.1].

(2) The Torelli curve complex in [51] (see also [101]), its vertices are homotopy classes of separating simple closed curves of $S_{g}$ and bounding pairs of curves. It plays a similar role for the Torelli group as the curve 
complex $\mathcal{C}\left(S_{g, n}\right)$ for the mapping class group $\operatorname{Mod}_{g, n}$, which is equal to $\operatorname{Diff}^{+}\left(S_{g, n}\right) / \operatorname{Diff}^{0}\left(S_{g, n}\right)$ (see $\$ 7.5$ below).

(3) The pants complex in [74] whose vertices are pants decompositions of $S_{g, n}$. It was originally used to find generators and relations for the mapping class group $\operatorname{Mod}_{g, n}$. It was also used to describe the coarse geometry of the Weil-Petersson metric of Teichmüller space [33]. (Note that the pants complex is a CW-complex but not a simplicial complex.)

(4) The train-track complex in [65] [143]. It was used in [65] to prove quasiisometric rigidity of the mapping class $\operatorname{group} \operatorname{Mod}_{g, n}$ (see Proposition 7.15 below).

(5) Sub-complexes of $\mathcal{C}\left(S_{g, n}\right)$ such as the complex of separating curves [145] [115] [101] (see also [72]).

(6) The complex of domains and its various subcomplexes [129]. ([129] contains a comprehensive list of complexes associated to surfaces.)

(7) A family of complexes related to the curve complex [136].

Remark 3.1. A class of groups closely related to arithmetic groups and mapping class groups consists of outer automorphism groups $\operatorname{Out}\left(F_{n}\right)$ of the free group $F_{n}$ on $n$ generators. There are several candidates for the analogue of the spherical Tits building $\Delta_{\mathbb{Q}}(\mathbf{G})$ and the curve complex $\mathcal{C}\left(S_{g, n}\right)$. They are infinite simplicial complexes on which $\operatorname{Out}\left(F_{n}\right)$ acts simplically, and they are also homotopy equivalent to a bouquet of spheres. See [75] [99] [88].

\section{Geometric and topological properties of buildings}

As mentioned in the introduction, the original motivation of Tits buildings was to give a geometric interpretation of exceptional Lie groups and hence to construct their analogues over finite fields. Their geometric and topological structures have been used for various applications, some of which will be explained in this chapter.

First we define canonical metrics on buildings, their diameters, and curvature properties. Then we state the Solomon-Tits Theorem which determines the topology of buildings. Finally we explain a relation between Euclidean buildings and spherical buildings.

Recall that a geodesic segment in a metric space $(M, d)$ is an isometric embedding $\gamma:[a, b] \rightarrow M$. $(M, d)$ is called a geodesic metric space if every two points in $M$ are joined by a geodesic segment. $M$ is called geodesically complete if every geodesic segment in $M, \gamma:[a, b] \rightarrow M$, can be extended infinitely in both directions to a map $\gamma:(-\infty,+\infty)$ such that for any $t \in(-\infty,+\infty)$, 
when $\left|t-t^{\prime}\right|$ is sufficiently small, $d\left(\gamma\left(t^{\prime}\right), \gamma(t)\right)=\left|t-t^{\prime}\right|$, i.e., the map is only locally distance minimizing. Note that this is different from the notion of a complete geodesic metric space. Clearly any complete Riemannian manifold is geodesically complete. On the other hand, any Riemannian manifold with nonempty boundary is not geodesically complete, since a geodesic segment perpendicular to the boundary cannot be extended.

Proposition 4.1. Every spherical Tits building $\Delta$ admits a metric such that

(1) its restriction to each apartment is isometric to the unit sphere $S^{r-1}$ in $\mathbb{R}^{r}$, where $r$ is the rank of the building, i.e., the number of vertices of the top dimensional simplices;

(2) it is a complete geodesic metric space;

(3) it is geodesically complete.

This metric is called the Tits metric. If $\Delta=\Delta(G)$, then $G$ acts simplicially and isometrically on $\Delta(G)$.

The idea of the proof is as follows. By definition, each apartment of $\Delta$ is a finite Coxeter complex, which is a triangulation of the unit sphere $S^{r-1}$. The metric on $S^{r-1}$ induces a geodesic metric on each apartment. The axioms for buildings show that the metrics on all the apartments are compatible, for example they agree on intersection, and they can be patched together to form a metric on $\Delta$. Since all apartments are isometric and can be mapped isometrically from one to another, it can be shown that $\Delta$ is a geodesic metric space. Since each apartment is geodesically complete, it can be shown similarly that $\Delta$ is also geodesically complete.

Proposition 4.2. For every spherical Tits building $\Delta$, its diameter with respect to the Tits metric is equal to $2 \pi$.

Proof. It is clear that the diameter of every apartment is equal to $2 \pi$. Since every two points of $\Delta$ are contained in an apartment, the diameter of $\Delta$ is less than or equal to $2 \pi$. Since any apartment can be retracted to a fixed apartment, it can be shown that each apartment is a totally geodesic subspace and hence the diameter of $\Delta$ is equal to $2 \pi$.

Proposition 4.3. Every Euclidean building $\Delta$ admits a metric such that

(1) its restriction to each apartment is isometric to the Euclidean space $\mathbb{R}^{r}$, where $r$ is the rank of the building, i.e., $r$ is equal to the dimension of the top dimensional simplices of $\Delta$, or equivalently, $r+1$ is the number of vertices of the top dimensional simplices of the Euclidean building $\Delta_{;}^{3}$

\footnotetext{
${ }^{3}$ It might be worthwhile to emphasize that the rank of a spherical Tits building in Proposition 4.1 is equal to 1 plus the dimension of the top dimensional simplices. This convention
} 
(2) it is a complete geodesic metric space;

(3) it is geodesically complete;

(4) with respect to this metric, $\Delta$ is a CAT(0)-space in the sense that every two distinct points are connected by a unique geodesic, and every triangle in $\Delta$ is thinner than the corresponding geodesic in $\mathbb{R}^{r}$ of the same side lengths.

This metric is called the Tits metric. If $\Delta=\Delta^{B T}(\mathbf{G})$, the Bruhat-Tits building of a linear semisimple algebraic group defined over a non-Archimedean local field $k$, then $G(k)$ acts isometrically on $\Delta$.

See [2] for more details and also [30] for a general discussion on CAT(0)spaces.

Corollary 4.4. The diameter of any Euclidean building with respect to the Tits metric is infinite.

Proof. This follows from the fact that every apartment is a totally geodesic subspace and hence has infinite diameter.

Proposition 4.5. Every hyperbolic building $\Delta$ admits a metric such that

(1) its restriction to each apartment is isometric to the hyperbolic space $\mathbf{H}^{n}$;

(2) it is a complete geodesic metric space;

(3) it is geodesically complete;

(4) with respect to this metric, $\Delta$ is a CAT(-1)-space in the sense that every two distinct points are connected by a unique geodesic, and every triangle in $\Delta$ is thinner than the corresponding geodesic in $\mathbf{H}^{n}$ of the same side lengths;

(5) it has infinite diameter.

This metric is called the Tits metric on $\Delta$.

Recall that a geodesic metric space is called a $\delta$-hyperbolic space if for every triangle in the space, any one side is contained in the $\delta$-neighborhood of the union of the other two sides. It is known that the real hyperbolic

is different from the convention of rank of Euclidean buildings here. Roughly speaking, the reason for these conventions is that the rank of buildings should be equal to the rank of the algebraic groups which define them. For example, a simple algebraic group over $\mathbb{R}$ of rank 1 gives a 0 -dimensional Tits building, and a simple algebraic group defined over a $p$-adic number field of rank 1 gives a 1-dimensional Euclidean building. Both buildings are defined to have rank 1. Another reason for such conventions is that for each Euclidean building of rank $r$, there is a spherical building of the same rank $r$ which can be added to the infinity of the Euclidean building. See $\S 6.8$ below. 
space $\mathbf{H}^{n}$ is $\delta$-hyperbolic. More generally, every simply connected Riemannian manifold with sectional curvature bounded from above by a negative constant is also $\delta$-hyperbolic, and every $C A T(-1)$ geodesic metric space is $\delta$-hyperbolic. Every metric tree is 0 -hyperbolic (i.e., $\delta$-hyperbolic, where $\delta=0$ if we use closed $\delta$-neighborhoods.) Note that every metric space with a finite diameter is automatically hyperbolic for trivial reasons. On the other hand, when $n \geq 2$, $\mathbb{R}^{n}$ is not $\delta$-hyperbolic.

Corollary 4.6. Every hyperbolic building is $\delta$-hyperbolic.

Proposition 4.7. For a Euclidean building $\Delta$, if its rank is equal to 1, then it is a tree and is a $\delta$-hyperbolic space, otherwise it is not a $\delta$-hyperbolic space.

Proof. The first statement was mentioned earlier, and the second statement follows from the fact that for any $\delta$-hyperbolic space, any totally geodesic subspace is also $\delta$-hyperbolic. (Recall that a subspace $Y$ of a geodesic space $X$ is called a totally geodesic subspace if for any two points $x, y \in Y$, any geodesic segment in $X$ connecting $x$ and $y$ is contained in $Y$.)

The topology of spherical buildings is given by the Solomon-Tits Theorem $[2]$.

Proposition 4.8. Let $\Delta$ be a spherical Tits building of rank $r$. Then $\Delta$ is homotopy equivalent to a bouquet of spheres $S^{r-1}$. If $\Delta=\Delta_{\mathbb{Q}}(\mathbf{G})$, then the bouquet contains infinitely many spheres.

Basically, fix one simplex $\sigma$ in $\Delta$; then $\Delta$ is the union of apartments containing $\sigma$. Each apartment is homotopy equivalent to a sphere, and this union gives the bouquet. When $\Delta=\Delta_{\mathbb{Q}}(\mathbf{G})$, there are infinitely many $\mathbb{Q}$-parabolic subgroups and hence there are infinitely many spheres in the bouquet.

On the other hand, the topology of Euclidean and hyperbolic buildings is trivial, i.e., they are contractible.

Proposition 4.9. Euclidean buildings and hyperbolic buildings are contractible.

Proof. By Proposition 4.3, every Euclidean building $\Delta$ is a CAT(0)-space. Fix a base point $x_{0} \in \Delta$. Then any other point $x \in \Delta$ can be connected to $x_{0}$ by a unique geodesic, and deformation retraction along such rays from $x_{0}$ shows that $\Delta$ is contractible. Since a CAT(-1)-space is also a CAT(0)-space (or by Proposition 4.5), the same proof works for hyperbolic buildings. 


\section{Geometric and topological properties of curve complexes}

The curve complex $\mathcal{C}\left(S_{g, n}\right)$ has some properties that are similar to all three types of buildings in the previous subsection.

We can put a metric on $\mathcal{C}\left(S_{g, n}\right)$ by making each simplex a standard Euclidean one with side length 1.

Proposition 5.1. $\mathcal{C}\left(S_{g, n}\right)$ is a complete geodesic space, i.e., it is a geodesic space and is complete as a metric space.

To prove this proposition, we need mapping class groups and their actions on $\mathcal{C}\left(S_{g, n}\right)$. Let $\operatorname{Diff}^{+}\left(S_{g, n}\right)$ be the group of orientation preserving diffeomorphisms of $S_{g, n}$, and $\operatorname{Diff}^{0}\left(S_{g, n}\right)$ its identity component, which is a normal subgroup. $\operatorname{Diff}^{+}\left(S_{g, n}\right) / \operatorname{Diff}^{0}\left(S_{g, n}\right)$ is called the mapping class group of $S_{g, n}$ and denoted by $\operatorname{Mod}_{g, n}$. A closely related group is the extended mapping class group $\operatorname{Mod}_{g, n}^{ \pm}=\operatorname{Diff}\left(S_{g, n}\right) / \operatorname{Diff}^{0}\left(S_{g, n}\right)$, where $\operatorname{Diff}\left(S_{g, n}\right)$ is the group of all diffeomorphisms of $S_{g, n}$ including both orientation preserving and orientation reversing diffeomorphisms.

When $3 g-3+n \geq 2$, for any essential simple closed curve $c$ on $S_{g, n}$, there are infinitely many essential simple closed curves that are disjoint from $c$ and not homotopy equivalent to $c$. This implies that the curve complex $\mathcal{C}\left(S_{g, n}\right)$ is not locally finite. Gluing up the metrics on the simplices certainly gives a length metric on the space, but it is not obvious why it is a geodesic metric, i.e., every two points are connected by a geodesic segment. This follows from a theorem on metric simplicial complexes in [29, Theorem 1.1].

Proposition 5.2. If $K$ is a metric simplicial complex with only finitely many isometry types of simplices, then the metric on $K$ glued up from the metrics of the simplices is a complete geodesic metric space.

To apply Proposition 5.2 to prove Proposition 5.1, we need to show that $\mathcal{C}\left(S_{g, n}\right)$ has a large symmetry group.

Proposition 5.3. The mapping class group $\operatorname{Mod}_{g, n}$ and the extended mapping class group $\operatorname{Mod}_{g, n}^{ \pm}$act simplicially and isometrically on $\mathcal{C}\left(S_{g, n}\right)$ with respect to the metric which is glued up from the simplices, and the quotients $\operatorname{Mod}_{g, n} \backslash \mathcal{C}\left(S_{g, n}\right)$ and $\operatorname{Mod}_{g, n}^{ \pm} \backslash \mathcal{C}\left(S_{g, n}\right)$ are finite $C W$-complexes, i.e., unions of finitely many cells.

Proof. Since diffeomorphisms of $S_{g, n}$ map simple closed curves to simple closed curves, preserve the homotopy classes, and preserve existence of disjoint representatives, it is clear that $\operatorname{Mod}_{g, n}$ acts simplicially on $\mathcal{C}\left(S_{g, n}\right)$. Since a 
top dimensional simplex of $\mathcal{C}\left(S_{g, n}\right)$ corresponds to a pants decomposition of $S_{g, n}$, the statement that $\operatorname{Mod}_{g, n} \backslash \mathcal{C}\left(S_{g, n}\right)$ is a finite complex is equivalent to the statement that there are only finitely many nonisomorphic pants decompositions of $S_{g, n}$. The latter is reduced to finiteness of the set of homeomorphism classes of trivalent graphs on the surface. See $[40, \S 3.5]$ for detail. The proof for $\operatorname{Mod}_{g, n}^{ \pm}$is the same.

\section{Proof of Proposition 5.1.}

By combining Propositions 5.2 and 5.3, we can see that $\mathcal{C}\left(S_{g, n}\right)$ is a complete geodesic space. (Proposition 5.1 was known and stated in [132, p. 1007].)

Remark 5.4. Since the curve complex $\mathcal{C}\left(S_{g, n}\right)$ is a thick chamber complex if $g \geq 2[69$, Proposition on page 266] in the sense that every co-dimension 1 simplex is contained in at least three top dimensional simplices, it might be reasonable to conjecture that $\mathcal{C}\left(S_{g, n}\right)$ is a geodesically complete metric space, i.e., every geodesic segment in it can be extended infinitely in both directions.

An important metric property of $\mathcal{C}\left(S_{g, n}\right)$ is the next result [127, Theorem 1.1, Proposition 4.6].

Proposition 5.5. The curve complex $\mathcal{C}\left(S_{g, n}\right)$ is a $\delta$-hyperbolic space of infinite diameter.

For simplified proofs, see [23] [63] [64]. As a $\delta$-hyperbolic space, $\mathcal{C}\left(S_{g, n}\right)$ has a boundary $\partial \mathcal{C}\left(S_{g, n}\right)$, which is similar to the geodesic boundary $X(\infty)$ of a symmetric space of noncompact type mentioned earlier and consists of equivalence classes of quasi-geodesics. (Note that for a proper $\delta$-hyperbolic space, the boundary can also be defined as the set of equivalence classes of geodesics [98, Proposition 2.10].)

Proposition 5.6. The boundary $\partial \mathcal{C}\left(S_{g, n}\right)$ is naturally homeomorphic to the space $\mathcal{E} \mathcal{L}\left(S_{g, n}\right)$ of laminations of $S_{g, n}$ which are filling and minimal (every leaf is dense in the support).

Proposition 5.6 is due to Klarreich [103]. See also [63] for a proof. For every complete hyperbolic metric of finite volume on $S_{g, n}$, a geodesic lamination is a closed subset which is a disjoint union of complete simple geodesics. A geodesic lamination is called filling if it intersects every simple closed geodesics. The set of filling geodesic laminations has a natural topology, which is the induced topology from the quotient of Thurston's topology. It is known that different choices of hyperbolic metrics on $S_{g, n}$ give rise to homeomorphic spaces of filling geodesic laminations. Therefore, for the surface $S_{g, n}$ of negative Euler characteristic, there is a well-defined space of filling and minimal laminations up to homeomorphism, denoted by $\mathcal{E} \mathcal{L}\left(S_{g, n}\right)$ in the proposition. 
A large scale invariant of a noncompact metric space is the asymptotic dimension introduced by Gromov. Another large scale invariant (or rather property) is Property A, which is a weak amenability-type condition. See [140] for definition.

Proposition 5.7. The asymptotic dimension of $\mathcal{C}\left(S_{g, n}\right)$ is finite and has Property A.

The former was proved in [8] and the latter in [100]. For some results on the quasi-isometry type of $\mathcal{C}\left(S_{g, n}\right)$, see [148].

The homotopy type of $\mathcal{C}\left(S_{g, n}\right)$ was determined in [67] (see also [66] and [80]).

Proposition 5.8. The curve complex $\mathcal{C}\left(S_{g, n}\right)$ is homotopy equivalent to a bouquet of spheres $S^{d}$, where $d=2 g-2=-\chi\left(S_{g}\right)$ if $n=0, d=-\chi\left(S_{g, n}\right)-1=$ $2 g-3+n$ if $g \geq 1$ and $n>0$, and $d=-\chi\left(S_{0, n}\right)-2=n-4$ if $g=0$.

The natural question on how many spheres in the bouquet was answered in $[86]$.

Proposition 5.9. The curve complex $\mathcal{C}\left(S_{g, n}\right)$ has infinite topology, i.e., the bouquet of spheres in Proposition 5.8 contains infinitely many spheres.

Propositions 5.8 and 5.9 are an analogue of the Solomon-Tits theorem for spherical buildings (Proposition 4.8).

Remark 5.10. Apartments are special and important subcomplexes of buildings and they explain easily the Solomon-Tits theorem. Though the curve complex $\mathcal{C}\left(S_{g, n}\right)$ shares many properties with buildings, one important difference or rather a mystery is that inside $\mathcal{C}\left(S_{g, n}\right)$, in general, there are no known corresponding distinguished finite subcomplexes whose underlying spaces are spheres or are homotopy equivalent to spheres.

In the special case $g=2, n=1$, spheres in $\mathcal{C}\left(S_{g, n}\right)$ that generate the top dimensional homology group have been constructed in [31, §4.3]. There are also some candidates of spheres in $\mathcal{C}\left(S_{g, n}\right)$ when $n=1$ in $[31, \S 4.3]$.

It might be helpful to note that $\mathcal{C}\left(S_{g, n}\right)$ contains many infinite subcomplexes corresponding to the curve complexes of sub-surfaces of $S_{g, n}$. These subcomplexes have played an important role in understanding the large scale geometry of $\mathcal{C}\left(S_{g, n}\right)$ and $\mathcal{T}_{g, n}$ in [125] [126]. 


\section{Selected applications of buildings}

There are many applications of buildings. We select some which are similar to the applications of curve complexes discussed below for the purpose of comparison. For more applications of buildings, see [87].

\subsection{Automorphism groups of buildings}

One reason why buildings are useful in geometry and topology is that they are rigid and also admit a large symmetry group.

We state briefly several general results for spherical Tits buildings. For the precise statements, see Theorems and Propositions numbered by 5.9, 6.3, 6.13, 8.4.5, 9.1, 10.2 in $[166,5.8]$.

Recall that a building is called thick if every simplex of codimension 1 is contained in at least three top dimensional simplices.

Proposition 6.1. Every thick irreducible spherical Tits building of rank at least 3 is the spherical building of a linear semisimple algebraic group.

Proposition 6.2. If $\Delta_{k}(\mathbf{G})$ and $\Delta_{k^{\prime}}\left(\mathbf{G}^{\prime}\right)$ are irreducible thick spherical Tits buildings of rank at least 2 associated with linear semisimple algebraic groups $\mathbf{G}$ and $\mathbf{G}^{\prime}$ defined over fields $k$ and $k^{\prime}$ respectively, then any isomorphism between $\Delta_{k}(\mathbf{G})$ and $\Delta_{k^{\prime}}\left(\mathbf{G}^{\prime}\right)$ is essentially determined by an isomorphism between the algebraic groups $\mathbf{G}$ and $\mathbf{G}^{\prime}$ and an isomorphism between $k$ and $k^{\prime}$.

See also [138, Theorem 16.1, Corollary 16.2].

Corollary 6.3. If $\Delta_{k}(\mathbf{G})$ is an irreducible thick spherical Tits building of rank at least 2 associated with a semisimple linear algebraic group defined over a field $k$, then any automorphism of $\Delta_{k}(\mathbf{G})$ is essentially induced by automorphisms of the group $\mathbf{G}$ and the field $k$.

\subsection{Mostow strong rigidity and generalizations}

One major application of Tits buildings is Mostow strong rigidity (also called Mostow-Prasad strong rigidity) for locally symmetric spaces [138] [144].

Let $X=G / K$ be a symmetric space of noncompact type. Any discrete subgroup $\Gamma \subset G$ acts properly on $X$ and the quotient $\Gamma \backslash X$ is called a locally symmetric space. The invariant metric on $X$ defines an invariant measure on $X$ which induces a measure on $\Gamma \backslash X$. $\Gamma$ is called a lattice if the volume of $\Gamma \backslash X$

with respect to the induced measure is finite. If $G=\mathbf{G}(\mathbb{R})$ is the real locus of a linear semisimple algebraic group and $\Gamma \subset \mathbf{G}(\mathbb{R})$ is an arithmetic subgroup 
(see the next subsection for a precise definition), then $\Gamma$ is a lattice in $G$, by the reduction theory of arithmetic subgroups.

A locally symmetric space $\Gamma \backslash X$ is called irreducible if no finite cover splits isometrically as a product.

Theorem 6.4 (Mostow strong rigidity). Let $\Gamma \backslash X$ and $\Gamma^{\prime} \backslash X^{\prime}$ be two irreducible locally symmetric spaces of finite volume. Assume that one of the symmetric spaces $X$ and $X^{\prime}$ is not isometric to the hyperbolic plane $\mathbf{H}^{2}$, and $\Gamma$ and $\Gamma^{\prime}$ are isomorphic as abstract groups. Then $\Gamma \backslash X$ and $\Gamma^{\prime} \backslash X^{\prime}$ are isometric after suitable scaling of the metrics on the irreducible factors of $X$ and $X^{\prime}$.

We note that if $\Gamma$ is torsion-free, then $\Gamma$ acts on $X$ fixed-point freely, and $\Gamma \backslash X$ is a manifold and its fundamental group $\pi_{1}(\Gamma \backslash X)$ is equal to $\Gamma$. Otherwise, $\Gamma \backslash X$ is an orbifold, and its fundamental group as an orbifold is equal to $\Gamma$. Mostow strong rigidity says roughly that if a locally symmetric space of finite volume is not a hyperbolic surface, then its isometry type is determined by its fundamental group.

The proof in [138] depends on the rank of $X$ and works under the assumption that $\Gamma \backslash X$ and $\Gamma^{\prime} \backslash X^{\prime}$ are compact. The basic idea is that an isomorphism between $\Gamma$ and $\Gamma^{\prime}$ induces an equivariant quasi-isometry between $X$ and $X^{\prime}$. When the rank of $X$ is at least 2, it induces an isomorphism between the Tits buildings $\Delta(X)$ and $\Delta\left(X^{\prime}\right)$, which are of rank at least 2, and the rigidity of Tits buildings in $\S 6.1$ implies the desired rigidity. The rank 1 case requires a different proof that involves quasi-conformal maps and ergodic actions of lattices on the sphere $X(\infty)$, which is a real analytic manifold in this case.

The remaining cases where $\Gamma \backslash X$ and $\Gamma^{\prime} \backslash X^{\prime}$ are noncompact were proved in [144] when the rank of $X$ is equal to 1 , and in [123] when the rank of $X$ is at least 2 .

Remark 6.5. Since the original proofs in [138], there have been several different proofs of Mostow strong rigidity. When the locally symmetric spaces are irreducible and the covering symmetric spaces are of rank at least 2, the result also follows from the stronger super-rigidity of Margulis [124]. When $\Gamma \backslash X$ and $\Gamma^{\prime} \backslash X^{\prime}$ are compact, there are also proofs of Mostow strong rigidity in some cases by the method of harmonic maps (see [59] for a summary and references).

When the rank of $X$ is equal to 1 , in particular when $X$ is the real hyperbolic space, there are at least two completely new proofs:

(1) A proof by Gromov using the notion of simplicial volume and the fact that the simplicial volume of a finite volume hyperbolic manifold is proportional to the volume of the hyperbolic metric. See the books [10] and [149] for detailed descriptions. 
(2) A proof in [13] using the notion of barycenter map that characterizes locally symmetric spaces among compact negatively curved Riemannian manifolds in terms of minimal entropy.

There are also proofs in [165] [84] of Mostow strong rigidity for hyperbolic spaces using quasi-conformal maps that generalize and simplify the original proof in [138].

Mostow strong rigidity has been generalized in several different ways. One generalization in [4] replaces one locally symmetric space, say $\Gamma^{\prime} \backslash X^{\prime}$, by a compact Riemannian manifold $M^{\prime}$ of nonpositive curvature, and still concludes that if $\Gamma \backslash X$ and $M^{\prime}$ are homotopy equivalent, $\Gamma \backslash X$ is irreducible and $X$ is of rank at least 2, then $M^{\prime}$ is also a locally symmetric space isometric to $\Gamma \backslash X$ after a scaling of the metric. A further generalization in [109] replaces the above Riemannian manifold $M^{\prime}$ by a geodesically complete metric space whose universal covering space is a $\mathrm{CAT}(0)$-space without changing the above conclusion. For this generalization, the following characterization of symmetric spaces of noncompact type and Euclidean buildings was proved in [109, Main Theorem 1.2].

Proposition 6.6. Let $X$ be a locally compact geodesically complete CAT(0)space. Let $d_{\text {Tits }}$ be the Tits metric on $X(\infty)$. If $\left(X(\infty), d_{\text {Tits }}\right)$ is isomorphic to a connected thick irreducible spherical building of rank at least 2 with the Tits metric, then $X$ is either a Riemannian symmetric space of noncompact type or a Euclidean building.

Another type of rigidity problems concerns the characterization of locally symmetric spaces in terms of intrinsic geometric properties. For example, the rank rigidity of nonpositively curved Riemannian manifolds in [3] [38] says that any irreducible nonpositively curved Riemannian manifold $M$ of finite volume with rank at least 2 is a locally symmetric space. The proof of [38] consists of two steps:

(1) The construction of a topological Tits building structure on $\widetilde{M}(\infty)$, where $\widetilde{M}$ is the universal covering space of $M$,

(2) The use of rigidity and classification of topological buildings in [39].

All these rigidity results have one thing in common: the asymptotic geometry of the spaces at infinity is described by Tits buildings, and rigidity of Tits buildings implies the desired rigidity of the spaces.

\subsection{Compactifications of locally symmetric spaces}

Let $\mathbf{G} \subset G L(n, \mathbb{C})$ be a linear semisimple algebraic group defined over $\mathbb{Q}$, and $G=\mathbf{G}(\mathbb{R})=\mathbf{G} \cap G L(n, \mathbb{R})$ the real locus, a real Lie group with finitely 
many connected components. Let $K \subset G$ be a maximal compact subgroup. Then $X=G / K$ with an invariant metric is a symmetric space of noncompact type. Let $\mathbf{G}(\mathbb{Q})=\mathbf{G} \cap G L(n, \mathbb{Q})$ be the rational locus of $\mathbf{G}$. A subgroup $\Gamma \subset \mathbf{G}(\mathbb{Q})$ is called an arithmetic subgroup if it is commensurable with $\mathbf{G}(\mathbb{Z})=$ $\mathbf{G}(\mathbb{Q}) \cap G L(n, \mathbb{Z})$, i.e., $\Gamma \cap \mathbf{G}(\mathbb{Z})$ has finite index in both $\Gamma$ and $\mathbf{G}(\mathbb{Z})$. By the reduction theory of arithmetic subgroups, it is known that $\Gamma \backslash X$ has finite volume and $\Gamma \backslash X$ is compact if and only if the $\mathbb{Q}$-rank $r_{\mathbb{Q}}(\mathbf{G})$ of $\mathbf{G}$ is equal to 0 , which is equivalent to the condition that there is no proper $\mathbb{Q}$-parabolic subgroup of $\mathbf{G}$.

For the rest of this subsection, we assume that $\Gamma \backslash X$ is noncompact unless otherwise indicated and let $\mathcal{P}_{\mathbb{Q}}$ be the collection of proper $\mathbb{Q}$-parabolic subgroups of $\mathbf{G}$.

For various applications, we need to compactify $\Gamma \backslash X$. We will discuss one compactification here. See [19] for other motivations and applications.

For any discrete group $\Gamma$, there is a classifying space $B \Gamma$ which is a CWcomplex satisfying the conditions: $\pi_{1}(B \Gamma)=\Gamma$ and $\pi_{i}(B \Gamma)=\{1\}$ when $i \geq 2$. Such a space $B \Gamma$ is unique up to homotopy equivalence. Its universal covering space $E \Gamma=\widetilde{B \Gamma}$ is a universal space for proper and fixed point free actions of $\Gamma$. $E \Gamma$ is a $\Gamma-C W$ complex characterized by the conditions: (1) $\Gamma$ acts properly and fixed point freely on $E \Gamma$, and (2) $E \Gamma$ is contractible.

It is known that if $\Gamma$ contains nontrivial torsion elements, then there does not exist finite-dimensional $B \Gamma$ or $E \Gamma$ spaces. In this case, another important space is the universal space for proper actions of $\Gamma$ which is usually denoted by $\underline{E} \Gamma$ and is characterized by the conditions: (1) $\underline{E} \Gamma$ is a $\Gamma-\mathrm{CW}$ complex and $\Gamma$ acts properly on it, (2) for any finite subgroup $F \subset \Gamma$, the fixed point set $(\underline{E} \Gamma)^{F}$ is nonempty and contractible. In particular, $\underline{E} \Gamma$ is contractible.

If $\Gamma$ is torsion-free, then the only finite subgroup of $\Gamma$ is the trivial one, and an $\underline{E} \Gamma$-space is an $E \Gamma$-space.

Definition 6.7. A classifying space $B \Gamma$ is called finite if it is a finite $C W$ complex. Equivalently, $E \Gamma$ is called cofinite if the quotient $\Gamma \backslash E \Gamma \cong B \Gamma$ is a finite $C W$-complex. Similarly, $\underline{E} \Gamma$ is called a cofinite universal space for proper actions of $\Gamma$ if $\Gamma \backslash \underline{E} \Gamma$ is a finite $C W$-complex.

For topological problems involving $\Gamma$, in particular cohomological properties and invariants of $\Gamma$, it is important to find explicit and cofinite models of $E \Gamma$ and $\underline{E} \Gamma[116]$.

Proposition 6.8. Let $\Gamma \subset \mathbf{G}(\mathbb{Q})$ be an arithmetic subgroup as above. Then

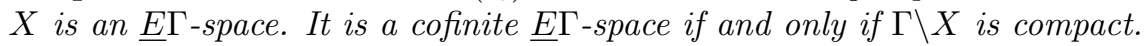

Proof. It is known that $X$ is a simply connected and nonpositively curved Riemannian manifold. For any finite subgroup $F \subset \Gamma$, by the Cartan fixed point theorem, the set of fixed points $X^{F}$ is nonempty. In fact, for any point 
$x \in X$, the so-called center of gravity of the orbit $F x$, which is finite, is fixed by $G$. Since $X^{F}$ is a totally geodesic submanifold, it is also contractible.

If $X$ is a cofinite $\underline{E} \Gamma$ space, then $\Gamma \backslash X$ is compact. Conversely, if $\Gamma \backslash X$ is compact, then the existence of an equivariant triangulation implies that $X$ has the structure of a $\Gamma$-CW-complex such that $\Gamma \backslash X$ is a finite $\mathrm{CW}$-complex.

When $\Gamma$ is torsion-free, then $\Gamma \backslash X$ is a $B \Gamma$-space. Assume that $\Gamma \backslash X$ is noncompact. One approach to obtain a compact $B \Gamma$-space is to construct a compactification $\overline{\Gamma \backslash X}$ such that the inclusion $\Gamma \backslash X \rightarrow \overline{\Gamma \backslash X}$ is a homotopy equivalence. The Borel-Serre compactification $\overline{\Gamma \backslash X}^{B S}$ in [20] satisfies this property.

For every $\mathbb{Q}$-parabolic subgroup $\mathbf{P} \in \mathcal{P}_{\mathbb{Q}}$, let $P=\mathbf{P}(\mathbb{R})$ be its real locus, $\mathbf{N}_{\mathbf{P}}$ be its unipotent radical, and $N_{\mathbf{P}}=\mathbf{N}_{\mathbf{P}}(\mathbb{R})$ its real locus. Let $\mathbf{A}_{\mathbf{P}}$ be a $\mathbb{Q}$-split component of $\mathbf{P}$ that is stable under the Cartan involution of $\mathbf{G}$ with respect to the maximal compact subgroup $K$ of $G$, and $A_{\mathbf{P}}=\mathbf{A}_{\mathbf{P}}(\mathbb{R})$ its real locus. Then $P$ admits a $\mathbb{Q}$-Langlands decomposition

$$
P=N_{\mathbf{P}} A_{\mathbf{P}} M_{\mathbf{P}} \cong N_{\mathbf{P}} \times A_{\mathbf{P}} \times M_{\mathbf{P}}
$$

The subgroup $M_{\mathbf{P}}$ is a reductive subgroup and $M_{\mathbf{P}} \cap K$ is a maximal compact subgroup of $M_{\mathbf{P}}$. The quotient $X_{\mathbf{P}}=M_{\mathbf{P}} / M_{\mathbf{P}} \cap K$ with an invariant metric induced from $X$ is a symmetric space of nonpositive sectional curvature (it might contain a flat factor and may not be of noncompact type), and is called the boundary symmetric space of $\mathbf{P}$. The $\mathbb{Q}$-Langlands decomposition of $P$ induces a horospherical decomposition of $X$ with respect to the $\mathbb{Q}$-parabolic subgroup P:

$$
X \cong N_{\mathbf{P}} \times A_{\mathbf{P}} \times X_{\mathbf{P}}
$$

One example to illustrate this is $\mathbf{G}=\mathrm{SL}(2, \mathbb{C})$, and $\Gamma=\mathrm{SL}(2, \mathbb{Z})$. Then

$$
\mathbf{P}=\left\{\left(\begin{array}{cc}
a & b \\
0 & a^{-1}
\end{array}\right) \mid a \in \mathbb{R}^{\times}, b \in \mathbb{R}\right\}
$$

is a $\mathbb{Q}$-parabolic subgroup, and

$$
N_{\mathbf{P}}=\left\{\left(\begin{array}{ll}
1 & b \\
0 & 1
\end{array}\right) \mid b \in \mathbb{R}\right\}, \quad A_{\mathbf{P}}=\left\{\left(\begin{array}{cc}
a & 0 \\
0 & a^{-1}
\end{array}\right) \mid a>0\right\}, \quad M_{\mathbf{P}}=\left\{\left(\begin{array}{cc}
a & 0 \\
0 & a^{-1}
\end{array}\right) \mid a= \pm 1\right\} .
$$

The boundary symmetric space $X_{\mathbf{P}}$ is one point, and the horospherical decomposition of $X=\mathbf{H}^{2}=\mathrm{SL}(2, \mathbb{R}) / \mathrm{SO}(2)$ with respect to $\mathbf{P}$ is the $(x, y)$ horospherical coordinates of the upper half plane $\mathbf{H}^{2}$. See [19] for more detail.

For every $\mathbb{Q}$-parabolic subgroup $\mathbf{P}$, define

$$
e(\mathbf{P})=N_{\mathbf{P}} \times X_{\mathbf{P}}
$$

and call it the boundary component of $\mathbf{P}$. 
According to the slightly modified procedure in [19], the Borel-Serre compactification $\overline{\Gamma \backslash X}^{B S}$ is constructed in the following steps.

(1) For every $\mathbf{P} \in \mathcal{P}_{\mathbb{Q}}$, attach the boundary symmetric space $e(\mathbf{P})$ at the infinity of $X$ using the horospherical decomposition of $X$ with respect to $\mathbf{P}$ to obtain a Borel-Serre partial compactification

$$
\overline{\mathbb{Q}}^{B S}=X \cup \coprod_{\mathbf{P} \in \mathcal{P}_{\mathbb{Q}}} e(\mathbf{P}) .
$$

(2) Show that the Borel-Serre partial compactification $\overline{\mathbb{Q}}^{B S}$ is a real analytic manifold with corners.

(3) Show that the $\Gamma$-action on $X$ extends to a proper real analytic action on $\overline{\mathbb{Q}}^{B S}$ with a compact quotient, which is the Borel-Serre compactification of $\Gamma \backslash X$ and denoted by $\overline{\Gamma \backslash X}^{B S}$.

From the above description, it is clear that the simplices of the Tits building $\Delta_{\mathbb{Q}}(\mathbf{G})$ parametrize the boundary components of the Borel-Serre compactification $\overline{\Gamma \backslash X}^{B S}$.

Corollary 6.9. When $\Gamma$ is torsion-free, $\overline{\Gamma \backslash X}^{B S}$ is a finite BГ-space.

Proof. When $\Gamma$ is torsion-free, it acts fixed point freely on $\overline{\mathbb{Q}}^{B S}$ and hence $\overline{\Gamma \backslash X}^{B S}$ is a real analytic compact manifold with corners whose interior is equal to $\Gamma \backslash X$. Then it is clear that the inclusion $\Gamma \backslash X \rightarrow \overline{\Gamma \backslash X}^{B S}$ is a homotopy equivalence by retracting from the boundary faces into the interior. Hence $\pi_{1}\left(\overline{\Gamma \backslash X}^{B S}\right)=\Gamma$ and $\pi_{i}\left(\overline{\Gamma \backslash X}^{B S}\right)=\{1\}$ for $i \geq 2$, and $\overline{\Gamma \backslash X}^{B S}$ is a $B \Gamma$ space. Since a compact manifold with corners admits a finite triangulation, $\overline{\Gamma \backslash X}^{B S}$ is a finite $B \Gamma$-space.

When $\Gamma$ contains torsion elements, the following result was proved in [89].

Proposition 6.10. The Borel-Serre partial compactification $\overline{\mathbb{Q}}^{B S}$ is a cofinite $\underline{E} \Gamma$-space.

The only non-obvious condition to check is that for any finite subgroup $F \subset \Gamma$, the fixed point set $\left(\overline{\mathbb{Q}}^{B S}\right)^{F}$ is contractible. The point is that if $F$ fixes a point in the boundary component $N_{\mathbf{P}} \times X_{\mathbf{P}}$, then $F$ is contained in $P$.

For applications in the next subsection, we note

Proposition 6.11. The boundary $\partial_{\mathbb{Q}} \bar{X}^{B S}$ is homotopy equivalent to the spherical Tits building $\Delta_{\mathbb{Q}}(\mathbf{G})$, and hence to a bouquet of infinitely many spheres $S^{r_{\mathbb{Q}}(\mathbf{G})-1}$, where $r_{\mathbb{Q}}(\mathbf{G})$ is the $\mathbb{Q}$-rank of $\mathbf{G}$. 
Proof. Since each boundary space $X_{\mathbf{P}}$ is contractible, and since for any two $\mathbb{Q}$-parabolic subgroups $\mathbf{P}_{1}, \mathbf{P}_{2}$, the inclusion relation $\mathbf{P}_{2} \subset \mathbf{P}_{2}$ holds if and only if $X_{\mathbf{P}_{1}}$ is contained in the closure of $X_{\mathbf{P}_{2}}$, the first statement follows. The second statement follows from Proposition 4.8 and the fact that $\Delta_{\mathbb{Q}}(\mathbf{G})$ is a spherical building of $\operatorname{rank} r_{\mathbb{Q}}(\mathbf{G})$.

Remark 6.12. To compare with the two partial compactifications of Teichmüller space in $\$ 7.5$, we mention that in the above construction of the Borel-Serre compactification, if we replace the boundary component $e(\mathbf{P})$ by the boundary symmetric space $X_{\mathbf{P}}$, then we obtain the reductive Borel-Serre partial compactification

$$
\overline{\mathbb{Q}}^{R B S}=X \cup \coprod_{\mathbf{P} \in \mathcal{P}_{\mathbb{Q}}} X_{\mathbf{P}} .
$$

Its boundary decomposes into contractible components $X_{\mathbf{P}}$, and these boundary components are also parametrized by the Tits building $\Delta_{\mathbb{Q}}(\mathbf{G})$ and its boundary $\partial \overline{\mathbb{Q}}^{R B S}$ is also homotopy equivalent to a bouquet of spheres $S^{r_{\mathbb{Q}}(\mathbf{G})-1}$. The $\Gamma$-action on $X$ also extends to a continuous action on $\overline{\mathbb{Q}}^{R B S}$ with a compact quotient $\Gamma \backslash \overline{\mathbb{Q}}^{R B S}$. All these results on the boundary of reductive Borel-Serre partial compactification are similar to the Borel-Serre partial compactification $\overline{\mathbb{Q}}^{B S}$. But the difference is that this extended action on $\overline{\mathbb{Q}}^{R B S}$ is not proper any more. The inclusion $\Gamma \backslash X \rightarrow \Gamma \backslash \overline{\mathbb{Q}}^{R B S}$ is not a homotopy equivalence. In some sense, $\overline{\Gamma \backslash X}^{B S}$ is a blow-up of the reductive Borel-Serre compactification $\Gamma \backslash \overline{\mathbb{Q}}^{R B S}$.

\subsection{Cohomological dimension and duality properties of arithmetic groups}

Let $\Gamma \subset \mathbf{G}(\mathbb{Q})$ be an arithmetic subgroup as in the previous subsection. Once good models of classifying spaces for $\Gamma$ such as $B \Gamma$ and $\underline{E} \Gamma$ spaces are found, they can be used to study cohomological properties of $\Gamma$.

Recall that for any discrete group $\Gamma$, the cohomological dimension $\operatorname{cd}(\Gamma)$ is defined by

$$
\mathrm{cd}(\Gamma)=\sup \left\{i \mid H^{i}(\Gamma, M) \neq 0, \text { for some } \mathbb{Z} \Gamma \text {-module } M\right\} .
$$

It is known that if $\Gamma$ contains nontrivial torsion elements, then $\operatorname{cd}(\Gamma)=+\infty$.

For any discrete group $\Gamma$ that is virtually torsion-free, i.e., if $\gamma$ contains a finite index torsion-free subgroup $\Gamma^{\prime}$, then $\operatorname{cd}\left(\Gamma^{\prime}\right)$ is independent of the choice of $\Gamma^{\prime}$ and is called the virtual cohomological dimension of $\Gamma$, denoted by $\operatorname{vcd}(\Gamma)$. It is also known that for any classifying space $B \Gamma^{\prime}, \operatorname{cd}\left(\Gamma^{\prime}\right) \leq \operatorname{dim} B \Gamma^{\prime}$. See [36] for more details. 
It is also known that every arithmetic subgroup $\Gamma \subset \mathbf{G}(\mathbb{Q})$ is virtually torsion-free. Since $X$ is an $E \Gamma$-space for a torsion-free arithmetic subgroup, an immediate corollary is the following bound on $\operatorname{vcd}(\Gamma)$ :

$$
\operatorname{vcd}(\Gamma) \leq \operatorname{dim} X \text {. }
$$

The precise value of $\operatorname{vcd}(\Gamma)$ was determined in [20] using the Borel-Serre partial compactification $\overline{\mathbb{Q}}^{B S}$ and the topology of the spherical Tits building $\Delta_{\mathbb{Q}}(\mathbf{G})$.

Proposition 6.13. The $\operatorname{vcd}(\Gamma)=\operatorname{dim} X-r_{\mathbb{Q}}(\mathbf{G})$, where $r_{\mathbb{Q}}(\mathbf{G})$ is the $\mathbb{Q}$-rank of $\mathbf{G}$, i.e., the maximal dimension of $\mathbb{Q}$-split tori in $\mathbf{G}$.

This was proved together with a stronger result on duality properties of $\Gamma$ in [20]. A group $\Gamma$ is called a Poincaré duality group of dimension $n$ if for every $\mathbb{Z} \Gamma$-module $M$ and every $i$, there is an isomorphism

$$
H^{i}(\Gamma, M) \cong H_{n-i}(\Gamma, M) .
$$

If $\Gamma$ admits a $B \Gamma$-space given by a closed orientable manifold of dimension $n$, then $\Gamma$ is a Poincaré duality group. But the converse is not true in general. An important question is under what further conditions a Poincaré duality group $\Gamma$ admits a closed manifold as a $B \Gamma$-space.

More generally, $\Gamma$ is called a duality group of dimension $n$ if there exists a $\mathbb{Z} \Gamma$-module $D$, called the dualizing module, such that for every $\mathbb{Z} \Gamma$-module $M$ and every integer $i$, there is an isomorphism

$$
H^{i}(\Gamma, M) \cong H_{n-i}(\Gamma, D \otimes M) .
$$

In this case, it is known that $\operatorname{cd}(\Gamma)=n$.

Since groups $\Gamma$ containing nontrivial torsion elements have $\operatorname{cd}(\Gamma)=+\infty$, they cannot be duality groups. On the other hand, if $\Gamma$ admits a finite-index torsion-free subgroup that is a duality group, it is called a virtual duality group. Similarly, the notion of virtual Poincaré duality group can be defined.

The stronger result proved in [20] is the following:

Proposition 6.14. Every arithmetic subgroup $\Gamma \subset \mathbf{G}(\mathbb{Q})$ as above is a virtual duality group of dimension $\operatorname{dim} X-r_{\mathbb{Q}}$, where $r_{\mathbb{Q}}=r_{\mathbb{Q}}(\mathbf{G})$. It is a Poincaré duality group if and only if $r_{\mathbb{Q}}=0$, i.e., $\Gamma \backslash X$ is compact. When $r_{\mathbb{Q}}>0$, the dualizing module of $\Gamma$ is equal to the $\mathbb{Z} \Gamma$-module $\bar{H}_{r_{\mathbb{Q}}-1}\left(\Delta_{\mathbb{Q}}(\mathbf{G})\right)$, where $\bar{H}_{*}$ is the reduced homology group.

The conclusion that $\Gamma$ is a virtual Poincaré duality group if $\Gamma \backslash X$ is compact is clear since it is a closed orientable manifold if $\Gamma$ is torsion-free.

Since $\mathbf{G}(\mathbb{Q})$ and hence $\Gamma$ acts on the set of $\mathbb{Q}$-parabolic subgroups of $\mathbf{G}$ and hence on the spherical Tits building $\Delta_{\mathbb{Q}}(\mathbf{G})$, for every $i, H_{i}\left(\Delta_{\mathbb{Q}}(\mathbf{G})\right)$ is a 
$\mathbb{Z} \Gamma$-module. By Proposition 4.8, the reduced homology of $\Delta_{\mathbb{Q}}(\mathbf{G})$ is non-zero in only degree $r_{\mathbb{Q}}(\mathbf{G})-1$.

The basic idea of the proof of Proposition 6.14 is as follows. Assume that $\Gamma$ is torsion-free and $\Gamma \backslash X$ is noncompact. Then $\overline{\Gamma \backslash X}^{B S}$ is a finite $B \Gamma$ space and $\overline{\mathbb{Q}}^{B S}$ is a cofinite $E \Gamma$-space. By general results for cohomology of groups [36, Proposition 7.5, Proposition 8.2, Theorem 10.1], it suffices to show that $H^{i}(\Gamma, \mathbb{Z} \Gamma) \cong H_{c}^{i}\left(\overline{\mathbb{Q}}^{B S}, \mathbb{Z}\right)$ is not zero only when $i=\operatorname{dim} X-r_{\mathbb{Q}}(\mathbf{G})$, where $H_{c}^{i}\left(\overline{\mathbb{Q}}^{B S}, \mathbb{Z}\right)$ denotes the cohomology group with compact support. By Poincaré-Lefschitz duality,

$$
H_{c}^{i}\left(\overline{\mathbb{Q}}^{B S}, \mathbb{Z}\right) \cong H_{d-i}\left(\overline{\mathbb{Q}}^{B S}, \partial \overline{\mathbb{Q}}^{B S}\right),
$$

where $d=\operatorname{dim} X$. Since $\overline{\mathbb{Q}}^{B S}$ is contractible,

$$
H_{d-i}\left(\overline{\mathbb{Q}}^{B S}, \partial \overline{\mathbb{Q}}^{B S}\right) \cong \bar{H}_{d-i-1}\left(\partial \overline{\mathbb{Q}}^{B S}\right),
$$

where $\bar{H}_{*}$ is the reduced homology. By Proposition 6.11 , the latter is not zero if and only if $d-i-1=r_{\mathbb{Q}}(\mathbf{G})-1$, i.e., $i=d-r_{\mathbb{Q}}(\mathbf{G})$. This proves that $\Gamma$ is a duality group of dimension $d-r_{\mathbb{Q}}(\mathbf{G})$ and the dualizing module is equal to $\bar{H}_{d-r_{\mathbb{Q}}(\mathbf{G})}\left(\Delta_{\mathbb{Q}}(\mathbf{G}), \mathbb{Z}\right)$.

\subsection{Simplicial volumes of locally symmetric spaces}

An important homotopy invariant of manifolds is the simplicial volume introduced by Gromov [58].

Suppose that $M$ is a connected oriented compact manifold of dimension $n$, let $[M]$ be the fundamental class in $H_{n}(M, \mathbb{R})$, or rather the image of the fundamental class in $H_{n}(M, \mathbb{Z})$ under the natural map $H_{n}(M, \mathbb{Z}) \rightarrow H_{n}(M, \mathbb{R})$. For each $n$-chain $c=\sum_{\sigma} a_{\sigma} \sigma$ with $\mathbb{R}$-coefficients, where $\sigma$ are $n$-singular simplices, define the simplicial $\ell^{1}$-norm

$$
\|c\|_{1}=\sum_{\sigma}|\sigma| .
$$

Then the simplicial volume of $M$, denoted by $\|M\|$, is defined by

$\|M\|=\inf \left\{\|c\|_{1} \mid c\right.$ is an $n$-chain with $\mathbb{R}$-coefficients in the fundamental class $\left.[M]\right\}$.

If $M$ is a connected non-orientable manifold, then let $\tilde{M}$ be its double cover and define $\|M\|=\frac{1}{2}\|\tilde{M}\|$. If $M$ is an oriented orbifold, then it has a fundamental class and hence the usual notion of simplicial volume. If $M$ admits a finite smooth cover $N$, then it also has a orbifold simplicial volume $\|M\|_{\text {orb }}=\|N\| / d$, where $d$ is the degree of the covering $N \rightarrow M$. It follows from the multiplicative property of simplicial volumes of manifolds that $\|M\|_{\text {orb }}$ is independent of the choice of a finite smooth cover $N$. It is known 
that

$$
\|M\|_{o r b} \geq\|M\|
$$

and the strict inequality can occur. See [91] for details.

Assume that $M$ is a connected orientable noncompact manifold of dimension $n$. Let $H_{*}^{\mathrm{lf}}(M, \mathbb{R})$ be the locally finite homology group of $M$. Let $[M]^{\mathrm{lf}}$ be the fundamental class in $H_{*}^{\text {lf }}(M, \mathbb{R})$. For any locally finite $n$-chain $c=\sum_{\sigma} a_{\sigma} \sigma$, define the simplicial norm $\|c\|_{1}=\sum_{\sigma}|\sigma|$ as above. Recall that to say that a chain is locally finite means that every compact subset of $M$ meets the images of only finitely many singular simplices $\sigma$ in the chain. Hence, $\|c\|_{1}$ could be equal to infinity.

The simplicial volume $\|M\|$ of a noncompact manifold $M$ is defined by $\|M\|=\inf \left\{\|c\|_{1} \mid c\right.$ is a locally finite $n$-chain in the fundamental class $\left.[M]^{l f}\right\}$.

One of the motivations of Gromov for introducing the simplicial volume was to give a lower bound on the minimal volume of a manifold $M$. Consider all complete Riemannian metrics $g$ on $M$ whose sectional curvature $K(g)$ satisfies the bound $|K(g)| \leq 1$ at all points. Let $\operatorname{Vol}(M, g)$ denote the volume of $M$ with respect to the metric $g$. Then the minimal volume of $M$ is defined by

$\operatorname{Min}-\operatorname{Vol}(M)=\{\inf (\operatorname{Vol}(M, g) \mid g$ is a complete metric, $|K(g)| \leq 1\}$.

Another major application of simplicial volume is a different proof by Gromov of Mostow strong rigidity for compact hyperbolic spaces of dimension at least 3 as mentioned above. See [10] [149] for detailed discussions.

A basic result in $[58, \S 0.5]$ states that there exists a universal constant $C_{n}$ only depending on the dimension $n$ such that

$$
\operatorname{Min}-\operatorname{Vol}(M) \geq C_{n}\|M\| \text {. }
$$

Therefore, a natural problem is to understand when the simplicial volume $\|M\|$ is equal to zero.

It is known that if a compact manifold $M$ admits a self-map of degree greater than or equal to 2 , then its simplicial volume $\|M\|=0$. If a noncompact manifold $M$ admits a proper self-map of degree greater than or equal to 2 , then $\|M\|=0$ or $\|M\|=+\infty$. As a consequence, the simplicial volumes of spheres and tori are equal to zero. It is also known that the simplicial volume of $\mathbb{R}^{n}$ is equal to 0 .

For spaces related to locally symmetric spaces, the following results on simplicial volume are known:

Proposition 6.15. If $M$ is a complete hyperbolic manifold of finite volume, then $\|M\|>0$. More generally, if $M$ admits a complete metric such that its sectional curvature $K$ is bounded uniformly between two negative constants, then $\|M\|>0$. 
This is a a result due to Thurston $[58, \S 0.3])$.

Proposition 6.16. If $M=\Gamma \backslash X$ is a compact locally symmetric space of noncompact type, then $\|M\|>0$.

This was conjectured by Gromov [58, p. 11] and proved in [107] and [37].

Proposition 6.17. If $M=\Gamma \backslash X$ is an arithmetic locally symmetric space whose $\mathbb{Q}$-rank, denoted by $r_{\mathbb{Q}}(\mathbf{G})$, is greater than or equal to 3, then $\|M\|=0$.

This vanishing result was proved in [114]. In the proof, Proposition 6.9 and Proposition 6.11 are used crucially. Briefly, a vanishing criterion [58, p. 58] was applied. In order to apply this, a suitable covering of $\Gamma \backslash X$ with multiplicity at most $\operatorname{dim} \Gamma \backslash X$ is needed. For this purpose, the conditions that the map $\pi_{1}^{\infty}(\Gamma \backslash X)=\pi_{1}\left(\overline{\Gamma \backslash X}^{B S}\right) \rightarrow \pi_{1}(\Gamma \backslash X)=\pi_{1}\left(\overline{\Gamma \backslash X}^{B S}\right)$ is injective and that the virtual cohomological dimension of $\Gamma$ is at most $\operatorname{dim} \Gamma \backslash X-2$ are needed. Since the virtual cohomological dimension of $\Gamma$ is equal to $\operatorname{dim} \Gamma \backslash X-r_{\mathbb{Q}}(\mathbf{G})$, the condition that $r_{\mathbb{Q}}(\mathbf{G}) \geq 3$ is more than enough. The assumption that $r_{\mathbb{Q}}(\mathbf{G}) \geq 3$ is needed to show that the boundary $\partial_{\mathbb{Q}} X^{B S}$, which is homotopy equivalent to a bouquet of spheres $S^{r_{Q}(\mathbf{G})-1}$, is simply connected.

If $r_{\mathbb{Q}}(\mathbf{G})=1$, this vanishing result does not hold in general. For example, it was proved in [113] that if $M$ is a Hilbert modular variety, then $\|M\|>0$. Note that Hilbert modular varieties are important examples of locally symmetric spaces of $\mathbb{Q}$-rank 1. If the rank of $X$ is equal to 1 and $\Gamma \backslash X$ is noncompact, which implies that $r_{\mathbb{Q}}(\mathbf{G})=1$, then the sectional curvature of $\Gamma \backslash X$ is bounded by two negative constants and hence the simplicial volume of $\Gamma \backslash X$ is positive $[58, \S 0.3]$.

Remark 6.18. For any topological space $M$ of dimension $n$ that admits a suitable fundamental class in $H_{n}(M, \mathbb{Z})$ or $H_{n}^{\mathrm{lf}}(M, \mathbb{Z})$, we can define its simplicial volume. We can show that for any arithmetic locally symmetric space $\Gamma \backslash X$ of $\mathbb{Q}$-rank at least 3, and any irreducible arithmetic locally symmetric space $\Gamma \backslash X$ of $\mathbb{Q}$-rank at least 1 and the rank of $X$ at least 2, the simplicial volumes of the reductive Borel-Serre compactification and the Baily-Borel compactification (if $\Gamma \backslash X$ is Hermitian) also vanish. See [91] for details.

\subsection{Asymptotic cones of symmetric spaces and locally symmetric spaces}

Buildings also occur naturally in the large scale geometry of symmetric spaces $X$ and locally symmetric spaces $\Gamma \backslash X$.

For any metric space $(M, d)$, choose a basepoint $x_{0} \in X$. For any $\varepsilon>0$, consider the family of pointed metric spaces $\left(M, \varepsilon d, x_{0}\right)$. The $\operatorname{limit}_{\varepsilon \rightarrow 0}\left(M, \varepsilon d, x_{0}\right)$, 
if it exists, is called the asymptotic cone (or tangent cone at infinity) of $M$ and denoted by Cone $_{\infty}(M)$. Though the ordinary limits many not exist, there are always ultra limits, which may not be unique. Any Cone $_{\infty}(M)$ is a metric cone and does not depend on the choice of the basepoint $x_{0}$.

If $(M, d)$ has finite diameter, then $\mathrm{Cone}_{\infty}(M)$ consists of one point. If $M=\mathbb{R}^{n}$ with the standard Euclidean metric, then Cone $_{\infty}\left(\mathbb{R}^{n}\right)$ is isomorphic to $\mathbb{R}^{n}$ due to the scaling invariance of the Euclidean metric. On the other hand, if $M=\mathbf{H}^{n}, n \geq 2$, $\operatorname{Cone}_{\infty}(M)$ is an $\mathbb{R}$-tree that branches at every point. Recall that a usual simplicial tree branches only at a discrete set of points on every geodesic.

The asymptotic cone of a general symmetric space of noncompact type was determined in [104].

Proposition 6.19. If $X$ is a symmetric space of noncompact type, then $\mathrm{Cone}_{\infty}(X)$ is an $\mathbb{R}$-Euclidean building.

One application of this result is the quasi-isometric rigidity of symmetric spaces [104] [48], which is a generalization of Mostow strong rigidity.

Proposition 6.20. Let $X$ and $X^{\prime}$ be two symmetric spaces of noncompact type that have no irreducible factors of rank 1 . If $X$ and $X^{\prime}$ are quasi-isometric, then they are isometric up to suitable scaling of the metrics on irreducible factors.

If $\Gamma \subset \mathbf{G}(\mathbb{Q})$ is an arithmetic subgroup and $\Gamma \backslash X$ is noncompact, then Cone $_{\infty}(\Gamma \backslash X)$ was determined in [76] [110] [94].

Proposition 6.21. If $\Gamma \backslash X$ is a noncompact arithmetic locally symmetric space, then the asymptotic cone at infinity Cone $_{\infty}(\Gamma \backslash X)$ is a metric cone over the finite complex $\Gamma \backslash \Delta_{\mathbb{Q}}(\mathbf{G})$.

Recall that $\Gamma$ acts on $\Delta_{\mathbb{Q}}(\mathbf{G})$ via the action on the set $\mathcal{P}_{\mathbb{Q}}$ of parabolic subgroups of $\mathbf{G}$. By the reduction theory of arithmetic subgroups, there are only finitely many $\Gamma$-conjugacy classes of proper $\mathbb{Q}$-parabolic subgroups and hence $\Gamma \backslash \Delta_{\mathbb{Q}}(\mathbf{G})$ is a finite simplicial complex.

\subsection{Applications of Euclidean buildings}

In the previous subsections, we have mainly concentrated on applications of spherical Tits buildings. Now we briefly consider some applications of Euclidean buildings.

A natural generalization of arithmetic subgroups is the class of $S$-arithmetic subgroups. Let $S=\left\{p_{1}, \cdots, p_{l}, \infty\right\}$, where $p_{1}, \cdots, p_{l}$ are prime distinct 
numbers, and $\mathbb{Z}\left[\frac{1}{p_{1}}, \cdots, \frac{1}{p_{l}}\right]$ be the ring of $S$-integers, denoted by $\mathbb{Z}_{S}$. For any linear semisimple algebraic group $\mathbf{G} \subset G L(n, \mathbb{C})$ defined over $\mathbb{Q}$, let $\mathbf{G}\left(\mathbb{Z}_{S}\right)=\mathbf{G}(\mathbb{Q}) \cap G L\left(n, \mathbb{Z}_{S}\right)$. Then a subgroup $\Gamma$ of $\mathbf{G}(\mathbb{Q})$ is called an $S$ arithmetic subgroup if it is commensurable with $\mathbf{G}\left(\mathbb{Z}_{S}\right)$.

It is known that under the diagonal embedding, $\Gamma$ is a discrete subgroup of $G \times \mathbf{G}\left(\mathbb{Q}_{p_{1}}\right) \times \cdots \times \mathbf{G}\left(\mathbb{Q}_{p_{k}}\right)$, where $G=\mathbf{G}(\mathbb{R})$ is the real locus of $\mathbf{G}$.

For each $p_{i}$, let $\Delta_{p_{i}}$ be the Bruhat-Tits building of the group $\mathbf{G}\left(\mathbb{Q}_{p_{i}}\right)$. Let $X=G / K$ be the symmetric space of noncompact type associated with $G$ as above. Define

$$
X_{S}=X \times \Delta_{p_{1}} \times \cdots \times \Delta_{p_{k}} .
$$

Then $\Gamma$ acts properly on $X_{S}$, since each $\mathbf{G}\left(\mathbb{Q}_{p_{i}}\right)$ acts properly on $\Delta_{p_{i}}$.

Proposition 6.22. Any $S$-arithmetic subgroup $\Gamma$ acts properly on $X_{S}$ and $X_{S}$ is an $\underline{E} \Gamma$-space.

The reason is that each $\Delta_{p_{i}}$ is a $\operatorname{CAT}(0)$-space and hence the product $X_{S}$ is also a CAT(0)-space. Since the Cartan fixed point theorem holds for CAT(0)spaces, for any finite subgroup $F \subset \Gamma$, the fixed point set $\left(X_{S}\right)^{F}$ is nonempty. It is also a totally geodesic subspace.

If the quotient $\Gamma \backslash X_{S}$ is compact, then $X_{S}$ is a cofinite $\underline{E} \Gamma$-space by [93]. Otherwise, we need an analogue of the Borel-Serre partial compactification

$$
\overline{\mathbb{Q}}^{X}{ }^{B S}=\overline{\mathbb{Q}}^{B S} \times \Delta_{p_{1}} \times \cdots \times \Delta_{p_{k}},
$$

on which $\Gamma$ acts properly with a compact quotient. See [89].

Remark 6.23. Bruhat-Tits buildings, or rather the Bruhat-Tits theory, have played an important role in the representation theory of reductive $p$-adic Lie groups. Due to the lack of knowledge of the author, we only mention one application which is connected with the fact that Bruhat-Tits buildings are CAT(0)-spaces. In [139], it was proved that for any Bruhat-Tits building $\Delta^{B T}(\mathbf{G}(k))$, and any $g \in \operatorname{Aut}\left(\Delta^{B T}(\mathbf{G}(k))\right)$, the displacement function

$$
d_{g}: \Delta^{B T}(\mathbf{G}(k)) \rightarrow \mathbb{R}_{\geq 0}, x \mapsto \operatorname{dist}(x, g x),
$$

is a convex function. This has important applications in representation theory. See [137] for detail.

Remark 6.24. On the set of vertices of a Bruhat-Tits building, there is also a combinatorial distance, which is induced from the distance on the 1-skeleton of the building. This has applications to arithmetic algebraic geometry. In [170], non-Archimedean intersection indices on projective spaces over a nonArchimedean local field of characteristic zero are expressed in terms of the combinatorial distance of the Bruhat-Tits building for $\mathbf{G}=\operatorname{PGL}(n)$. 


\subsection{Compactifications of Euclidean buildings}

It is clear from the definition that Euclidean buildings are noncompact, since each apartment is a closed noncompact subspace. For many purposes, Euclidean buildings play a similar role for $p$-adic Lie groups as symmetric spaces of noncompact type for noncompact real Lie groups. There has been a lot of work on compactifications of symmetric spaces motivated by applications in group theory, geometry, topology and analysis. See [19] for a history, more details and references.

By Proposition 4.3, every Euclidean building $\Delta$ is a CAT(0)-space. It is also known that a symmetric space of noncompact type with an invariant Riemannian metric is a complete nonpositively curved simply connected Riemannian manifold and is hence also a CAT(0)-space. This is one important common property shared by both Euclidean buildings and symmetric spaces.

It is known that for any $\mathrm{CAT}(0)$-space $\Delta$, its set of equivalence classes of geodesics $X(\infty)$ forms the geodesic (or visual) boundary of $\Delta$ as in the case of a symmetric space that we saw in $\S 2.1$. If $\Delta$ is a proper metric space, then there is a natural compact topology on $\Delta \cup \Delta(\infty)$ [30] [21]. This compactification $X \cup X(\infty)$ is called the geodesic compactification. The following result was also proved in [21].

Proposition 6.25. (1) If $\Delta$ is a Euclidean building, then its geodesic boundary $\Delta(\infty)$ has a natural structure of a spherical Tits building. If the rank of $\Delta$ is equal to $r$, then the rank of $\Delta(\infty)$ is also equal to $r$. (2) If $\Delta=\Delta^{B T}(\mathbf{G})$ is the Bruhat-Tits building of an algebraic group $\mathbf{G}$ over a locally compact local field $k$, then $\Delta=\Delta^{B T}(\mathbf{G})$ is locally compact, and $\Delta(\infty)$ is the spherical Tits building $\Delta_{k}(\mathbf{G})$. Furthermore, the geodesic compactification $\Delta^{B T}(\mathbf{G}) \cup$ $\Delta^{B T}(\mathbf{G})(\infty)$ is contractible.

This geodesic compactification explains a close relation between spherical and Euclidean buildings.

Suppose $\Delta_{1}, \Delta_{2}$ are two locally compact Euclidean buildings. Then there are at least two natural compactifications of the product $\Delta_{1} \times \Delta_{2}$.

The first one is obtained by taking the product of the geodesic compactifications $\Delta_{1} \cup \Delta_{1}(\infty)$ and $\Delta_{2} \cup \Delta_{2}(\infty)$,

$$
{\overline{\Delta_{1} \times \Delta_{2}}}^{\text {prod }}=\left(\Delta_{1} \cup \Delta_{1}(\infty)\right) \times\left(\Delta_{2} \cup \Delta_{2}(\infty)\right) .
$$

Note that the product $\Delta_{1} \times \Delta_{2}$ is also a CAT(0)-space. Then the geodesic compactiication gives another compactification of $\Delta_{1} \times \Delta_{2}$,

$$
\Delta_{1} \times \Delta_{2} \cup\left(\Delta_{1} \times \Delta_{2}\right)(\infty) .
$$

It is clear that these two compactifications are not isomorphic, and each of them is natural in its own way. It turns out that the second type of compactifications has an application to the Novikov conjecture in geometric topology 
for certain classes of $S$-arithmetic subgroups of algebraic groups. Let $\Gamma$ be an $S$-arithmetic subgroup of a linear algebraic group as in $\S 6.7$. Assume that $\Gamma$ acts cocompactly on $X_{S}=X \times \Delta_{p_{1}} \times \cdots \Delta_{p_{k}}$. By Proposition $6.22, X_{S}$ is a cocompact universal space for proper actions of $\Gamma$, i.e., a cocompact model of $\underline{E}$. Since $X$ is also a CAT(0)-space as mentioned above, the product $X_{S}$ is a $\mathrm{CAT}(0)$-space and hence admits a geodesic compactification. The $\Gamma$-action on $X_{S}$ extends continuously to the compactification. An important point of the geodesic compactification of $X_{S}$ is that the extended action of $\Gamma$ on the compactification is small at the boundary (or infinity). Then it follows from a general criterion for the validity of Novikov conjectures that the integral Novikov conjecture holds for $\Gamma$. See [92] [93] for precise statements of the Novikov conjecture, for the criterion and for other details.

On the other hand, if we take the product compactification of $X_{S}$ induced from compactifications of the factors, i.e., the product of $X \cup X(\infty), \Delta_{p_{1}} \cup$ $\Delta_{p_{1}}(\infty), \cdots, \Delta_{p_{k}} \cup \Delta_{p_{k}}(\infty)$, the action of $\Gamma$ might not be small at infinity, and it might not be used for proving the integral Novikov conjecture for $\Gamma$.

Motivated by many different compactifications of symmetric spaces of noncompact type (see [19]), there are also corresponding compactifications of Euclidean buildings. As discussed in $\S 2.2$, one important feature of rich structure of a symmetric space is reflected by the flat subspaces $F$ of maximal dimension in $X$, which determine the Tits building of $X$.

There are several ways to understand a compactification $\bar{X}$ of $X$, and they can be generalized to construct compactifications of Euclidean buildings:

(1) Decompose the boundary $\bar{X}-X$ into smaller subspaces which enjoy some natural structures.

(2) Determine the closure of maximal flat subspaces $F$ in the compactification $\bar{X}$ and the intersection pattern of these compactified flat subspaces, and recover the compactification of $X$ from the compactified flats.

(3) Interpret $X$ as a moduli space of certain objects and the boundary points in $\bar{X}-X$ as degenerate objects.

For the geodesic compactification $X \cup X(\infty)$ in $\S 2.1$, the boundary $X(\infty)$ is naturally decomposed into simplices and can be viewed as the underlying space of the Tits building of $X$. As pointed out earlier, a geodesic compactification can be constructed for every locally compact Euclidean building.

Another important compactification of $X$ is the maximal Satake compactification $\bar{X}_{\max }^{S}$, which is the maximal element in the partially ordered finite set of Satake compactifications of $X$. See [60]. Each maximal flat $F$ of $X$ has a natural decomposition by Weyl chambers and Weyl chamber faces. The closure of $F$ in $\bar{X}_{\max }^{S}$ is canonically homeomorphic to a polyhedron, its boundary is a finite cell complex dual to the Weyl chamber decomposition, and the compactification $\bar{X}_{\max }^{S}$ can be constructed by gluing these compactified flats $[60]$. 
It turns out a similar construction works for any Euclidean building $\Delta$ by gluing polyhedral compactifications of apartments of $\Delta$. See [108].

To explain Satake compactifications of symmetric spaces, we start with a special Satake compactification of the symmetric space $\mathrm{SL}(n, \mathbb{C}) / \mathrm{SU}(n)$. Let $\mathcal{H}_{n}$ be the real vector space of $(n \times n)$-Hermitian matrices, and $P\left(\mathcal{H}_{n}\right)$ the associated projective space. Then the map $A \mapsto A \bar{A}^{t}$ defines an embedding of $\mathrm{SL}(n, \mathbb{C}) / \mathrm{SU}(n)$ into $P\left(\mathcal{H}_{n}\right)$, and the closure of $\mathrm{SL}(n, \mathbb{C}) / \mathrm{SU}(n)$ under this embedding gives a Satake compactification. In this case, every point in $\mathrm{SL}(n, \mathbb{C}) / \mathrm{SU}(n)$ corresponds to a positive definite Hermitian matrix of determinant 1 (or equivalently a positive definite Hermitian quadratic form of determinant 1), and the boundary points correspond to projective classes of degenerate positive semi-definite Hermitian matrices.

For a general symmetric space $X=G / K$ of noncompact type, any finitedimensional projectively faithful representation of $G$ gives an isometric embedding of $X$ into $\mathrm{SL}(n, \mathbb{C}) / \mathrm{SU}(n)$ for some $n$, and the closure of $X$ in the above special Satake compactification of $\mathrm{SL}(n, \mathbb{C}) / \mathrm{SU}(n)$ gives a Satake compactification of $X$. Though there are infinitely many representations of $G$, it turns out that they only give rise to finitely many non-isomorphic Satake compactifications of $X$.

For the Bruhat-Tits building $\Delta^{B T}(\mathbf{G})$ of a reductive algebraic group over a local field, the corresponding Satake compactifications have been constructed in [151] [150] [171] [172] [173]. The paper [150] constructs the compactification by using Berkovich analytic geometry over complete non-Archimedean fields, and the paper [151] uses irreducible representations of the algebraic group and is more similar to the Satake compactifications of symmetric spaces. The construction in [171] is also similar to the Satake compactifications of symmetric spaces. Compactifications of some special buildings were treated in [172] and [173].

The boundary $\partial \bar{X}_{\max }^{S}$ of the maximal Satake compactification is naturally decomposed into symmetric spaces of noncompact type of smaller dimension, which are naturally parametrized by proper parabolic subgroups of $G$, where $X=G / K$, or by simplices of the Tits building $\Delta(X)$. Since each boundary symmetric space is a cell, this shows that the boundary $\partial \bar{X}_{\max }^{S}$ has a natural cell structure which is dual to the Tits building. See [60]. The boundary $\partial \bar{X}_{\max }^{S}$ can also be constructed in this way by adding these boundary components using the idea of the Borel-Serre compactification of locally symmetric spaces. See [19]. For Bruhat-Tits buildings, a similar construction should also work. For the Bruhat-Tits building of $\operatorname{PGL}(n)$ over a local compact field, such a construction has been carried out in [171].

The maximal Satake compactification $\bar{X}_{\max }^{S}$ can also be constructed by embedding $X$ into the compact space of closed subgroups of $G$ [60]. A similar compactification of Bruhat-Tits buildings has been constructed in [61]. 
An important application of compactifications of symmetric spaces is to harmonic analysis on symmetric spaces, for example, the determination of the Poisson boundary. The analogue of the Poisson boundary of certain BruhatTits buildings has been determined in [57].

Remark 6.26. For a hyperbolic building $\Delta$, its geodesic boundary $\Delta(\infty)$ has the structure of a trivial (or rank 1) spherical building structure, i.e., the top dimensional simplices of the building are points. The reason is that the boundary $\mathbf{H}^{n}(\infty)$ of the real hyperbolic space $\mathbf{H}^{n}$ has the structure of a rank-1 spherical building.

\section{Applications of curve complexes}

In this section, we briefly discuss some applications of curve complexes. In $\S 7.1$, we discuss the identification of the automorphism group of $\mathcal{C}\left(S_{g, n}\right)$ with $\operatorname{Mod}_{g, n}$, which is responsible for several rigidity results on the geometry of $\mathcal{T}_{g, n}$ in $\S 7.2$. In $\S 7.3$, we briefly describe the ending lamination conjecture of Thurston on the rigidity of 3-dimensional hyperbolic manifolds with finitely generated fundamental group and its formulation in terms of the boundary $\partial \mathcal{C}\left(S_{g, n}\right)$ of $\mathcal{C}\left(S_{g, n}\right)$ as a $\delta$-hyperbolic space. This is probably one of the most striking applications of the curve complex $\mathcal{C}\left(S_{g, n}\right)$. In $\S 7.4$, we discuss an application to quasi-isometric rigidity of $\operatorname{Mod}_{g, n}$. In $\S 7.5$, we describe several different notions of the Novikov conjectures and an application of the curve complex to finiteness of the asymptotic dimension of $\operatorname{Mod}_{g, n}$. In $\S 7.6$, we mention an application to non-Gromov-hyperbolicity of the Weil-Petersson metric of $\mathcal{T}_{g, n}$. In $\S 7.7$, we define the Hempel distance of a Heegaard splitting via the image of Heegaard diagrams in $\mathcal{C}\left(S_{g}\right)$. After these applications to 3dimensional manifolds, we describe the Borel-Serre partial compactification of $\mathcal{T}_{g, n}$ via $\mathcal{C}\left(S_{g, n}\right)$, the original motivation of introducing $\mathcal{C}\left(S_{g, n}\right)$. Cohomological properties of $\operatorname{Mod}_{g, n}$ are studied in $\S 7.9$. The asymptotic cone at infinity of $\mathcal{M}_{g, n}$ is described in $\S 7.10$ and the simplicial volume of $\mathcal{M}_{g, n}$ is discussed in $\S 7.11$. In the last subsection, $\S 7.12$, we mention applications of $\mathcal{C}\left(S_{g, n}\right)$ to the classification of elements of $\operatorname{Mod}_{g, n}$, presentations and unitary representations of this group.

\subsection{Automorphism groups of curve complexes}

As mentioned in $\S 5$, the extended mapping class group $\operatorname{Mod}_{g, n}^{ \pm}$acts on $\mathcal{C}\left(S_{g, n}\right)$ simplicially. This defines a map $\operatorname{Mod}_{g, n}^{ \pm} \rightarrow \operatorname{Aut}\left(\mathcal{C}\left(S_{g, n}\right)\right)$, where $\operatorname{Aut}\left(\mathcal{C}\left(S_{g, n}\right)\right)$ is the simplicial automorphism group of $\mathcal{C}\left(S_{g, n}\right)$. It turns out that the image is essentially the whole automorphism group [81] [117, Theorem, p. 7] [105]. 
Proposition 7.1. (1) If the dimension of $\mathcal{C}\left(S_{g, n}\right)$ is at least 1 and $(g, n) \neq$ $(1,2)$, then the map $\operatorname{Mod}_{g, n}^{ \pm} \rightarrow \operatorname{Aut}\left(\mathcal{C}\left(S_{g, n}\right)\right)$ is surjective. (2) When $(g, n)=$ $(1,2)$, the image of the $\operatorname{map} \operatorname{Mod}_{g, n} \rightarrow \operatorname{Aut}\left(\mathcal{C}\left(S_{g, n}\right)\right)$ contains all automorphisms of $\mathcal{C}\left(S_{1,2}\right)$ that preserve vertices represented by separating curves. Furthermore, this map is injective and the image has index 2 in $\operatorname{Aut}\left(\mathcal{C}\left(S_{g, n}\right)\right)$. (3) When $S_{g, n}$ is not a torus with 1 or 2 punctures, or a sphere with at most 4 punctures, or a closed surface of genus 2, then the $\operatorname{map} \operatorname{Mod}_{g, n}^{ \pm} \rightarrow$ $\operatorname{Aut}\left(\mathcal{C}\left(S_{g, n}\right)\right)$ is an isomorphism.

This is an analogue of Corollary 6.3 for Tits buildings and it will be used in the next subsection to prove several rigidity results.

Remark 7.2. There are several related results on rigidity of simplicial maps of $\mathcal{C}\left(S_{g, n}\right)$ which imply co-Hopfian property of the mapping class group $\operatorname{Mod}_{g, n}$ [79] [162] [9]. Some quasi-isometric embeddings of curve complexes are constructed in [147], and they lead to some new quasi-isometric embeddings between mapping class groups.

Remark 7.3. A closely related result on the simplicial automorphism group of the arc complex of a surface is proved in [106]. The automorphism group of the pants complex of a surface is determined in [122].

Remark 7.4. The paper [17] studies the automorphism group Aut $\left(\hat{\mathcal{C}}\left(S_{g, n}\right)\right)$ of the profinite completion of the complex of curves $\mathcal{C}\left(S_{g, n}\right)$ and compares it with the Grothendieck-Teichmüller group. This paper also shows that $\operatorname{Aut}\left(\hat{\mathcal{C}}\left(S_{g, n}\right)\right)$ coincides (apart from two low dimensional exceptions) with the automorphism group of $\hat{\operatorname{Mod}}_{g, n}$, where $\hat{\operatorname{Mod}}_{g, n}$ is the profinite completion of the extended mapping class group $\operatorname{Mod}_{g, n}$.

\subsection{Isometry groups of Teichmüller spaces}

Motivated by the proof of Mostow strong rigidity for irreducible compact locally symmetric spaces of rank at least 2 via rigidity of Tits buildings, Ivanov [82] gave a new proof of the following theorem of Royden [154] by using the rigidity of $\mathcal{C}\left(S_{g, n}\right)$ in Proposition 7.1.

Proposition 7.5. The Teichmüller metric $d_{T e i}$ of the Teichmüller space $\mathcal{T}_{g, n}$ is invariant under the natural action of the extended mapping class group

$\operatorname{Mod}_{g, n}^{ \pm}$. If $g \geq 2$, or $g=1$ and $n \geq 3$, or $g=0$ and $n \geq 5$, then $\operatorname{Mod}_{g, n}^{ \pm}$is equal to the full isometry group $\operatorname{Iso}\left(\mathcal{T}_{g, n}, d_{T e i}\right)$.

The Teichmüller metric is historically the first metric defined on $\mathcal{T}_{g, n}$. It is a complete Finsler metric and it gives the natural topology on $\mathcal{T}_{g, n}$. The 
Teichmüller space $\mathcal{T}_{g, n}$ also admits another metric, the Weil-Petersson metric $d_{W P}$. This is an incomplete Kähler metric. Its isometry group was identified in $[128]$.

Proposition 7.6. For any Teichmüller space $\mathcal{T}_{g, n}$, the extended mapping class group $\operatorname{Mod}_{g, n}^{ \pm}$is canonically mapped into the isometry group $\operatorname{Iso}\left(\mathcal{T}_{g, n}, d_{W P}\right)$. If $g \geq 2$, or $g=1$ and $n \geq 3$, or $g=0$ and $n \geq 5$, then $\operatorname{Mod}_{g, n}^{ \pm}$is equal to the full isometry group $\operatorname{Iso}\left(\mathcal{T}_{g, n}, d_{W P}\right)$.

The idea of the proof, simplified in [174], is similar to the previous proposition and can be explained as follows. The completion of $\mathcal{T}_{g, n}$ in the WeilPetersson metric is the augmented Teichmüller space $\hat{\mathcal{T}}_{g, n}$. The boundary components of $\hat{\mathcal{T}}_{g, n}$ are Teichmüller spaces of stable Riemann surfaces of smaller genus with more punctures (the same Euler characteristic). These boundary components are parametrized by simplices of $\mathcal{C}\left(S_{g, n}\right)$. Since any isometry of $\left(\mathcal{T}_{g, n}, d_{W P}\right)$ extends to its completion, it induces an automorphism of $\mathcal{C}\left(S_{g, n}\right)$. Proposition 7.1 implies that the isometry is induced by an element of $\operatorname{Mod}_{g, n}$.

Remark 7.7. Teichmüller spaces $\mathcal{T}_{g, n}$ are the counterpart of symmetric spaces in the analogy between Tits buildings and arithmetic groups on the one hand, and curve complexes and mapping class groups on the other hand. The above proposition shows one difference. Any symmetric space is a homogeneous space and has continuous symmetry, but $\mathcal{T}_{g, n}$ has only discrete symmetry, which implies that there is a uniform lower bound on volumes of $\Gamma \backslash \mathcal{T}_{g, n}$. Maybe one result to repair this difference is that for any symmetric space $X$, there is a uniform lower bound on volumes of all locally symmetric spaces $\Gamma \backslash X$ which are quotients of $X$.

\subsection{The ending lamination conjecture of Thurston}

Another generalization of Mostow strong rigidity is the ending lamination conjecture of Thurston.

A special case of Mostow strong rigidity concerns 3-dimensional hyperbolic manifolds of finite volume. Let $\Gamma_{1} \backslash \mathbf{H}^{3}$ and $\Gamma_{2} \backslash \mathbf{H}^{3}$ be two hyperbolic manifolds of finite volume. If $\Gamma_{1} \cong \Gamma_{2}$, then $\Gamma_{1} \backslash \mathbf{H}^{3}$ and $\Gamma_{2} \backslash \mathbf{H}^{3}$ are isometric.

On the other hand, the conclusion does not hold if the volumes of $\Gamma_{1} \backslash \mathbf{H}^{3}$ and $\Gamma_{2} \backslash \mathbf{H}^{3}$ are not finite. For example, let $\Gamma \subset \mathrm{SL}(2, \mathbb{R})$ be a torsion-free cocompact Fuchsian group, i.e., a discrete subgroup such that $\Gamma \backslash \mathbf{H}^{2}$ is a compact hyperbolic surface $\Sigma$. Consider $\Gamma$ as a discrete subgroup of $\operatorname{SL}(2, \mathbb{C})$. Then $\Gamma \backslash \mathbf{H}^{3}$ is a 3-dimensional hyperbolic manifold of infinite volume and is diffeomorphic to $\Sigma \times \mathbb{R}$. There are injective morphisms $\rho: \Gamma \rightarrow \operatorname{SL}(2, \mathbb{C})$ such that $\rho(\Gamma) \backslash \mathbf{H}^{3}$ is not isometric to $\Gamma \backslash \mathbf{H}^{3}$, but is still diffeomorphic to $\Sigma \times \mathbb{R}$. 
A deformation $\rho(\Gamma)$ of $\Gamma$ is called a quasi-Fuchsian deformation if $\rho(\Gamma)$ is a quasi-Fuchsian group in the sense that the limit set of $\rho(\Gamma)$ in $\mathbf{H}^{3}(\infty)$ is a Jordan curve. The complement of the limit set consists of two simply connected domains $\Omega_{1}$ and $\Omega_{2}$. Many quasi-Fuchsian deformations of $\Gamma$ have the property that $\rho(\Gamma) \backslash \mathbf{H}^{3}$ is not isometric to $\Gamma \backslash \mathbf{H}^{3}$.

In this case, clearly $\Gamma$ and $\rho(\Gamma)$ are isomorphic. Therefore, a direct generalization of Mostow strong rigidity does not hold for infinite volume hyperbolic manifolds of dimension 3, i.e., if $\Gamma_{1} \backslash \mathbf{H}^{3}$ and $\Gamma_{2} \backslash \mathbf{H}^{3}$ are both of infinite volume, then $\Gamma_{1} \cong \Gamma_{2}$ does not imply that $\Gamma_{1} \backslash \mathbf{H}^{3}$ and $\Gamma_{2} \backslash \mathbf{H}^{3}$ are isometric. (Note that by [119, Theorem 8.3], if one of the manifolds $\Gamma_{1} \backslash \mathbf{H}^{3}$ and $\Gamma_{2} \backslash \mathbf{H}^{3}$ has finite volume and $\Gamma_{1} \cong \Gamma_{2}$, then the other manifold also has finite volume and hence $\Gamma_{1} \backslash \mathbf{H}^{3}$ and $\Gamma_{2} \backslash \mathbf{H}^{3}$ are isometric.)

For each quasi-Fuchsian deformation $\rho(\Gamma)$, the noncompact infinite volume 3-dimensional hyperbolic manifold $\rho(\Gamma) \backslash \mathbf{H}^{3}$ has two ends ${ }^{4}$ and admits a natural compactification $\overline{\rho(\Gamma) \backslash \mathbf{H}^{3}}$ by adding the two Riemann surfaces $\rho(\Gamma) \backslash \Omega_{1}$ and $\rho(\Gamma) \backslash \Omega_{2}$ as the boundary. Therefore, the Riemann surfaces $\rho(\Gamma) \backslash \Omega_{1}$ and $\rho(\Gamma) \backslash \Omega_{2}$ are called the Riemann surfaces at infinity of the ends of $\rho(\Gamma) \backslash \mathbf{H}^{3}$.

The following fact establishes a connection between the Riemann surfaces at infinity and the geometry of the interior.

Proposition 7.8. Two quasi-Fuchsian deformations $\rho_{1}(\Gamma)$ and $\rho_{2}(\Gamma)$ of $\Gamma$ give rise to isometric 3-dimensional hyperbolic manifolds $\rho_{1}(\Gamma) \backslash \mathbf{H}^{3}$ and $\rho_{2}(\Gamma) \backslash \mathbf{H}^{3}$ if and only if they have the same Riemann surfaces at infinity of the ends. Furthermore, all possible conformal structures on the Riemann surfaces at infinity can arise.

This is a proper generalization of Mostow strong rigidity for the class of quasi-Fuchsian hyperbolic manifolds, and it gives a complete classification of quasi-Fuchsian hyperbolic manifolds of dimension 3. See [12] [121, Chapter 5] for more details.

Quasi-Fuchsian deformations are important examples of geometrically finite hyperbolic manifolds. Recall that a three-dimensional hyperbolic manifold $\Gamma \backslash \mathbf{H}^{3}$ is called geometrically finite if $\Gamma$ admits a finite-sided convex fundamental domain (see [26] for discussion of several equivalent definitions), which is also equivalent to the fact that $\Gamma \backslash \mathbf{H}^{3} \cup \Omega(\Gamma)$ is homeomorphic to $M-P$ where $M$ is a compact 3-manifold with boundary and $P$ is a finite collection of disjoint annuli and tori in $\partial M$, and $\Omega(\Gamma)$ is the largest open subset of $\mathbf{H}^{3}(\infty)$ where $\Gamma$ acts properly $[43$, p. 139].

A similar rigidity result and classification of hyperbolic metrics holds for geometrically finite hyperbolic manifolds $M$ of dimension 3 . Roughly speaking,

\footnotetext{
${ }^{4} \mathrm{By}$ an end of a 3-dimensonal manifold, we mean a connected unbounded component of the complement of a sufficiently large compact subset. Strictly speaking, an end is a limit of such unbounded connected components over an exhausting family of compact subspaces. For a precise definition, see [159].
} 
for each end of $M$, there is a Riemann surface at infinity. Such a collection of Riemann surfaces at infinity together with the topology of the manifold uniquely determines the hyperbolic manifold [119, Theorem 8.1] [120] [121, Chapter 5] [12] [132, p. 188] [43, §7.3]. We note that the topology of a quasi-Fuchsian hyperbolic manifold is determined by any of the two Riemann surfaces at infinity, and hence the condition on the topology of the manifold is contained in the Riemann surfaces at infinity.

For a finitely generated torsion-free subgroup $\Gamma \subset \mathrm{SL}(2, \mathbb{C})$, the hyperbolic manifold $\Gamma \backslash \mathbf{H}^{3}$ has only finitely many ends. This case is more complicated than the geometrically finite case. For simplicity, in the following discussion, we assume that $\Gamma$ does not contain any nontrivial parabolic element (see [121, $\S 5.5]$ for the complication caused by the presence of parabolic elements.)

A three-dimensional hyperbolic manifold $M=\Gamma \backslash \mathbf{H}^{3}$ is called geometrically tame if each of its ends is either geometrically finite or simply degenerate (defined below). It is called topologically tame if $M$ is homeomorphic to the interior of a compact manifold with boundary. It is known that if $\Gamma$ is finitely generated, then $\Gamma \backslash \mathbf{H}^{3}$ is topologically tame if and only if it is geometrically tame [41] [18]. Therefore, the solution of Marden's tameness conjecture implies that $\Gamma \backslash \mathbf{H}^{3}$ is geometrically tame. See [42] for a precise description of these two notions of tameness and a history of Marden's tameness conjecture.

Thurston associated an invariant to an incompressible end of $\Gamma \backslash \mathbf{H}^{3}$, and an end invariant for a general end was defined in [41] (see also [18]).

If an end is geometrically finite and of infinite volume, then there is a Riemann surface at infinity which gives the end invariant. For a geometrically finite end of finite volume, there is no invariant.

For a simply degenerate end, there is a filling lamination on an associated surface, which is an invariant of the end. For simplicity, we assume that $\Gamma \backslash \mathbf{H}^{3}$ has no cuspidal ends. Then for each end, there is a surface $S_{g, n}$ such that the end is homeomorphic to $S_{g, n} \times(0,+\infty)$. For every simple closed curve $\alpha$ in $S_{g, n}$, there is a well-defined homotopy class of simple closed curves in the end of $\Gamma \backslash \mathbf{H}^{3}$ and hence in $\Gamma \backslash \mathbf{H}^{3}$. Let $\alpha^{*}$ be the unique geodesic representative in $\Gamma \backslash \mathbf{H}^{3}$. Then the end is called simply degenerate if there is a sequence of simple closed curves $\alpha_{i}$ on $S_{g, n}$ such that their geodesic representatives $\alpha_{i}^{*}$ exit the end, i.e., they are eventually contained in any small neighborhood of the end.

The family of curves $\alpha_{i}$ in $S_{g, n}$ converges to a lamination, called the ending lamination of the end. It can also be interpreted through the curve complex of a surface in the end. The sequence of simple closed curves $\alpha_{i}$ gives a sequence of vertices in the curve complex $\mathcal{C}\left(S_{g, n}\right)$. It is known that they converge to a boundary point $\partial \mathcal{C}\left(S_{g, n}\right)$, which is a filling lamination on $S_{g, n}$ by Proposition 5.6 .

Thurston made the following Ending lamination conjecture. 
Conjecture 7.9. A three dimensional hyperbolic manifold $\Gamma \backslash \mathbf{H}^{3}$ with finitely generated fundamental group $\Gamma$ is determined by its topological type and its end invariants.

If $\Gamma \backslash \mathbf{H}^{3}$ has finite volume, then each end has finite volume and is a cusp, the end invariant is trivial, and the space $\Gamma \backslash \mathbf{H}^{3}$ is determined by its topology, i.e., $\Gamma$ as the fundamental group, up to isometry by Mostow strong rigidity.

This ending lamination conjecture has been proved by Brock-Canary-Minsky (see [34] [130] [131]). Slightly different proofs have also been given. See [27] [28]. A crucial step in the proof is to build a bi-Lipschitz model of each of the ends of $\Gamma \backslash \mathbf{H}^{3}$. (In [27] [28], a weaker Lipschitz model of each end is needed and constructed.) The large-scale geometry of the curve complex $\mathcal{C}\left(S_{g, n}\right)$ and its connection with the Teichmüller metric of $\mathcal{T}_{g, n}$, in particular results in [126] [127], were used crucially for this purpose. (It seems that the papers [126] [127] were motivated by the ending lamination conjecture.) One result in [126] (see [64, Theorem 4.1]) says that when $\mathcal{T}_{g, n}$ is given the Teichmüller metric $d_{T e i}$, there is a $\operatorname{Mod}_{g, n}$-coarsely-equivariant map $\psi:\left(\mathcal{T}_{g, n}, d_{T e i}\right) \rightarrow \mathcal{C}\left(S_{g, n}\right)$ which is quasi-Lipschitz in the following sense:

(1) there exists a constant $c>1$ such that for every point $x \in \mathcal{T}_{g, n}$ and $\gamma \in \operatorname{Mod}_{g, n}$,

$$
d(\psi(\gamma x), \gamma \psi(x)) \leq c
$$

(2) for any two points $x, x^{\prime} \in \mathcal{T}_{g, n}$,

$$
d\left(\psi(x), \psi\left(x^{\prime}\right)\right) \leq c d_{T e i}\left(x, x^{\prime}\right)+c .
$$

This is an important instance of the philosophy that $\mathcal{C}\left(S_{g, n}\right)$ describes the large scale geometry of $\mathcal{T}_{g, n}$. For detailed descriptions of the ending lamination conjecture and methods to prove it, see [132] [133] [134] [27].

Remark 7.10. Besides giving a complete classification of 3-dimensional hyperbolic manifolds, the ending lamination conjecture has applications to the local connectivity of limit sets of Kleinian groups and the Cannon-Thurston map. See [135].

Remark 7.11. See [146] for related results on a model for the Teichmüller metric in terms of combinatorial information on short curves on the hyperbolic surfaces that correspond to points on a Teichmüller geodesic. The paper [102] compares the Thurston boundary of $\mathcal{T}_{g, n}$ and the boundary $\partial \mathcal{C}\left(S_{g, n}\right)$. 


\subsection{Quasi-isometric rigidity of mapping class groups}

In this subsection, we discuss another important application of curve complexes to quasi-isometric rigidity of $\operatorname{Mod}_{g, n}$. As mentioned before, the notion of quasiisometry was used in the proof of Mostow strong rigidity.

Recall that two metric spaces $\left(M_{1}, d_{1}\right)$ and $\left(M_{2}, d_{2}\right)$ are called quasi-isometric if there are positive constants $C, D$ and a map $f: M_{1} \rightarrow M_{2}$ such that for every two points $x_{1}, x_{2} \in M_{1}$,

$$
C^{-1} d_{2}\left(f\left(x_{1}\right), f\left(x_{2}\right)\right)-D \leq d_{1}\left(x_{1}, x_{2}\right) \leq C d_{2}\left(f\left(x_{1}\right), f\left(x_{2}\right)\right)+D,
$$

and every point of $M_{2}$ lies in the $D$-neighborhood of $f\left(M_{1}\right)$.

It is clear that if a finitely generated group $\Gamma$ acts properly and isometrically on a metric space $X$ with a compact quotient, then $\Gamma$ with any word metric is quasi-isometric to $X$. The quasi-isometry type of the group $\Gamma$ does not depend on the choice of a word metric.

A finitely generated group $\Gamma$ is called quasi-isometrically rigid if the following property holds: for any finitely generated group $\Gamma^{\prime}$, if $\Gamma^{\prime}$ is quasi-isometric to $\Gamma$, then there exists a finite-index subgroup $\Gamma^{\prime \prime} \subset \Gamma^{\prime}$ and a homomorphism $\Gamma^{\prime \prime} \rightarrow \Gamma$ with finite kernel and finite cokernel, i.e., $\Gamma^{\prime \prime}$ and $\Gamma$ are virtually isomorphic.

The three propositions below are combinations of results in several papers including [49] [48] [54] [160] [161] [104]. See [50] for the history, references and more detailed statements of these propositions. There are also other rigidity results on mapping class groups. See [80].

Proposition 7.12. If $\Gamma \subset G$ is an irreducible non-uniform lattice of a semisimple Lie group $G$, then $\Gamma$ is quasi-isometrically rigid.

The assumption that $\Gamma$ is not uniform is necessary. All uniform lattices of one semisimple Lie group $G$ are quasi-isometric, but they are not necessarily commensurable up to conjugation by elements of $G$, and hence they are not necessarily isomorphic up to finite index subgroups and quotients. On the other hand, this class of uniform lattices is quasi-isometrically rigid.

Proposition 7.13. If a finitely generated group $\Gamma^{\prime}$ is quasi-isometric to a uniform lattice in a semisimple Lie group $G$, then there exists a finite index subgroup $\Gamma^{\prime \prime}$ and a homomorphism $\rho: \Gamma^{\prime \prime} \rightarrow G$ with a finite kernel such that its image is a uniform lattice in $G$.

Proposition 7.14. Non-uniform lattices of $\mathrm{SL}(2, \mathbb{R})$ form one quasi-isometry class of groups.

Quasi-isometric rigidity and the quasi-isometric classification of lattices of semisimple Lie groups were obtained by joint efforts of many people. See [50] for the history and more detailed statements of the above three propositions. 
In the analogy between the mapping class groups $\operatorname{Mod}_{g, n}$ and lattices of semisimple Lie groups, $\operatorname{Mod}_{g, n}$ correspond to non-uniform lattices. Therefore, the next proposition on the quasi-isometrical rigidity of the mapping class group $\operatorname{Mod}_{g, n}$, proved in [65] [6], is expected.

Proposition 7.15. Assume that $3 g-3+n \geq 2$. Then $\operatorname{Mod}_{g, n}$ is quasiisometrically rigid.

One ingredient used in the proof in [6] is the hyperbolicity of the curve complex $\mathcal{C}\left(S_{g, n}\right)$ in the sense of Gromov, and one ingredient used in the proof of [65] is the train track complex. Results on the asymptotic cones at infinity Cone $_{\infty}\left(\operatorname{Mod}_{g, n}\right)$ in [7] (see Proposition 7.42 below) were used crucially.

The papers [65] [6] also prove the following result on quasi-isometries of $\operatorname{Mod}_{g, n}$.

Proposition 7.16. Assume that $3 g-3+n \geq 2$ and $(g, n) \neq(1,2)$, then quasi-isometries of $\operatorname{Mod}_{g, n}$ are uniformly close to isometries induced by leftmultiplication of elements of $\operatorname{Mod}_{g, n}$.

\subsection{Finite asymptotic dimension of mapping class groups and the Novikov conjectures}

In this subsection, we briefly describe another important application of the hyperbolicity of the curve complex $\mathcal{C}\left(S_{g, n}\right)$ to prove that the asymptotic dimension of the mapping class group $\operatorname{Mod}_{g, n}$ is finite. We also explain applications of this result to several different versions of the Novikov conjecture.

For any noncompact metric space $(M, d)$, an important large scale geometry invariant is the asymptotic dimension of $M$, denoted by asd $(M)$. It is defined to be the smallest integer $n$, which could be $\infty$, such that for every $r>0$, there exists a cover $\mathcal{C}=\left\{U_{i}\right\}, i \in I$, of $M$ by uniformly bounded sets $U_{i}$ with $r$-multiplicity less than or equal to $n+1$, i.e., every ball in $M$ of radius $r$ intersects at most $n+1$ sets in $\mathcal{C}$.

For any finitely generated group $\Gamma$, its asymptotic dimension $\operatorname{asd}(\Gamma)$ is defined to the asymptotic dimension of $\Gamma$ endowed with any word metric.

Finiteness of $\operatorname{asd}(\Gamma)$ has applications to the Novikov conjectures for $\Gamma$, including various versions of the integral Novikov conjectures. For the convenience of the reader, we briefly recall several versions of the Novikov conjectures, both the integral and rational versions. See [92] for more discussions, details and proper references for the conjectures and results stated below.

To motivate the Novikov conjectures, we first recall the Hirzebruch index theorem. Let $M^{4 k}$ be a compact oriented manifold (without boundary) of dimension $4 k$. The cup product defines a non-degenerate quadratic form on 
the middle dimension cohomology group:

$$
Q: H^{2 k}(M, \mathbb{Q}) \times H^{2 k}(M, \mathbb{Q}) \rightarrow H^{4 k}(M, \mathbb{Q})=\mathbb{Q} .
$$

This quadratic form can be diagonalized over $\mathbb{R}$ to the form $\operatorname{Diag}(1, \cdots, 1 ;-1, \cdots,-1)$, and the number of +1 's minus the number of -1 's is called the signature of $M$ and denoted by $\operatorname{Sgn}(M)$. Since the identification $H^{4 k}(M, \mathbb{Q})=\mathbb{Q}$ depends on the orientation of $M$, the signature $\operatorname{Sgn}(M)$ depends on the orientation and is an oriented homotopy invariant of $M$.

The Hirzebruch class $\mathcal{L}(M)$ is a power series in Pontrjagin classes $P_{1}, P_{2}, \cdots$, with rational coefficients,

$$
\mathcal{L}(M)=1+L_{1}+L_{2}+\cdots,
$$

where $L_{i}$ are polynomials of Pontrjagin classes, for example, $L_{1}=\frac{1}{3} P_{1}, \quad L_{2}=$ $\frac{1}{45}\left(7 P_{2}-P_{1}^{2}\right)$.

Then the Hirzebruch index theorem is the following equality:

$$
\operatorname{Sgn}(M)=\langle\mathcal{L}(M),[M]\rangle,
$$

where the right hand side is the evaluation of $\mathcal{L}(M)$ on the fundamental class $[M]$.

The Hirzebruch class $\mathcal{L}(M)$ depends on the characteristic classes of the tangent bundle of $M$ and a priori it also depends on the differentiable structure of $M$. (In fact, these rational classes in $H^{*}(M, \mathbb{Q})$ are homeomorphism invariants of $M)$. As pointed out earlier, the left-hand side in the above formula is an oriented homotopy invariant, and hence the above equality shows that $\langle\mathcal{L}(M),[M]\rangle$ only depends on the oriented homotopy type of $M$.

To get more homotopy invariants, Novikov introduced the higher signatures. Let $\Gamma=\pi_{1}(M)$. Let $B \Gamma$ be a classifying space of the discrete group $\Gamma$, i.e., a $K(\Gamma, 1)$-space,

$$
\pi_{1}(B \Gamma)=\Gamma, \quad \pi_{i}(B \Gamma)=\{1\}, \quad i \geq 2 .
$$

The universal covering space $E \Gamma$ of $B \Gamma$ is contractible and admits a free $\Gamma$ action. Equivalently, we can reverse this process and define first $E \Gamma$ as a contractible space with a free $\Gamma$-action, and then define $B \Gamma$ as the quotient $\Gamma \backslash E \Gamma$. For example, when $\Gamma=\mathbb{Z}$, it acts freely by translation on $\mathbb{R}$ and hence $E \Gamma=\mathbb{R}$ and $B \Gamma=\mathbb{R} / \mathbb{Z}=S^{1}$.

For each group $\Gamma$, the spaces $E \Gamma$ and $B \Gamma$ are unique up to homotopy. The universal covering map $\tilde{M} \rightarrow M$ determines a classifying map $f: M \rightarrow B \Gamma$, which is unique up to homotopy.

For any $\alpha \in H^{*}(B \Gamma, \mathbb{Q}), f^{*} \alpha \in H^{*}(M, \mathbb{Q})$, and define a higher signature

$$
\operatorname{Sgn}_{\alpha}(M)=\left\langle f^{*} \alpha \cup \mathcal{L}(M),[M]\right\rangle .
$$

The original Novikov conjecture is stated as follows: 
Conjecture 7.17 (Novikov conjecture). For any $\alpha \in H^{*}(B \Gamma, \mathbb{Q})$, the higher signature $\operatorname{Sgn}_{\alpha}(M)$ is an oriented homotopy invariant of $M$, i.e., if $N$ is another oriented manifold and $g: N \rightarrow M$ is an orientation preserving homotopy equivalence, then

$$
\left\langle(g \circ f)^{*} \alpha \cup \mathcal{L}(N),[N]\right\rangle=\left\langle f^{*} \alpha \cup \mathcal{L}(M),[M]\right\rangle .
$$

The Novikov conjecture can be reformulated in terms of the rational injectivity of the assembly map in surgery theory, or $L$-theory.

The surgery obstruction groups $L_{*}(\mathbb{Z}[\Gamma])$, or $L$-groups of $\mathbb{Z}[\Gamma]$, are briefly defined as follows. For $m=2 k, L_{m}(\mathbb{Z}[\Gamma])$ is the Witt group of stable isomorphism classes of $(-1)^{k}$-quadratic forms on finitely generated free modules over the group ring $\mathbb{Z}[\Gamma]$, and $L_{2 k+1}(\mathbb{Z}[\Gamma])$ is a stable automorphism group of hyperbolic $(-1)^{k}$-quadratic forms on finitely generated free modules over $\mathbb{Z}[\Gamma]$. Since $(-1)^{k}$ is 4-periodic in $m$, the groups $L_{m}(\mathbb{Z}[\Gamma])$ are 4-periodic in $m$.

Let $\mathbb{L}(\mathbb{Z})$ be the surgery spectrum:

$$
\pi_{m}(\mathbb{L}(\mathbb{Z}))=L_{m}(\mathbb{Z}), \quad m \in \mathbb{Z} .
$$

The spectrum $\mathbb{L}(\mathbb{Z})$ defines a general homology theory with coefficient in $\mathbb{L}(\mathbb{Z})$. For any topological space $X$, there are general homology groups $H_{*}(X ; \mathbb{L}(\mathbb{Z}))=$ $\pi_{*}\left(X_{+} \wedge \mathbb{L}(\mathbb{Z})\right)$, where $X_{+}$is the disjoint union of $X$ and a point.

There is an important notion of assembly map:

$$
A: H_{*}(X ; \mathbb{L}(\mathbb{Z})) \rightarrow L_{*}\left(\mathbb{Z}\left[\pi_{1}(X)\right]\right) .
$$

Proposition 7.18. The Novikov conjecture, i.e., the oriented homotopy invariance of the higher signatures in Conjecture 7.17, is equivalent to the rational injectivity of the assembly map in Equation (7.5.4), i.e., the following rational assembly map is injective:

$$
A \otimes \mathbb{Q}: H_{*}(B \Gamma ; \mathbb{L}(\mathbb{Z})) \otimes \mathbb{Q} \rightarrow L_{*}(\mathbb{Z}[\Gamma]) \otimes \mathbb{Q} .
$$

The injectivity of the map $A \otimes \mathbb{Q}$ is called the rational Novikov conjecture.

Conjecture 7.19 (Integral Novikov conjecture). If $\Gamma$ is torsion-free, then the assembly map $A: H_{*}(B \Gamma ; \mathbb{L}(\mathbb{Z})) \rightarrow L_{*}(\mathbb{Z}[\Gamma])$ is injective.

This conjecture is also called the L-theory (or surgery theory) integral Novikov conjecture.

In the integral Novikov conjecture, the torsion-free assumption on $\Gamma$ is important. In fact, it is known that the conjecture is often false for finite groups.

Clearly, the integral Novikov conjecture implies the rational Novikov conjecture and gives an integral version of homotopy invariance of higher signature. There are also several other reasons to consider the integral, rather than the original (rational) Novikov conjecture: 
(1) The relation to the rigidity of manifolds, in particular, the Borel conjecture for rigidity of aspherical manifolds, which says that two closed aspherical manifolds with the same fundamental group are homeomorphic.

(2) The computation of the L-groups $L_{*}(\mathbb{Z}[\Gamma])$ in terms of a generalized homology theory, i.e., the injectivity of the assembly map in Equation (7.5.4) shows that the left-hand side is a summand of the groups $L_{*}(\mathbb{Z}[\Gamma])$.

Once formulated in terms of the assembly map, there are also other versions of the Novikov conjecture. For any associative ring with unit $R$, there is a family of algebraic K-groups $K_{i}(R), i \in \mathbb{Z}$. For example, $K_{0}(R)$ is defined as the stable equivalence classes of finitely generated projective modules, and $K_{1}(R)=G L(R) /[G L(R), G L(R)]$. The higher K-groups $K_{i}(R), i \geq 2$, are defined to be the homotopy groups of the space $B G L(R)^{+}$, where $B G L(R)$ is the classifying space of $G L(R)$ considered as a discrete group, and $B G L(R)^{+}$is the space obtained by applying the Quillen +-construction to the perfect subgroup $E(R)=[G L(R), G L(R)]$, in particular, the homology groups of $B G L(R)^{+}$and $B G L(R)$ are equal to each other under inclusion. The K-theory spectrum $\mathbb{K}(R)$ with $\pi_{i}(\mathbb{K}(R))=K_{i}(R), i \in \mathbb{Z}$, is given by the delooping of the infinite loop space $B G L(R)^{+} \times K_{0}(R)$.

Let $\Gamma$ be a group as above, and $H_{*}(B \Gamma ; \mathbb{K}(\mathbb{Z}))$ the generalized homology of $B \Gamma$ with coefficients in $\mathbb{K}(R)$. There is also an assembly map

$$
A: H_{*}(B \Gamma ; \mathbb{K}(\mathbb{Z})) \rightarrow K_{*}(\mathbb{Z}[\Gamma]) .
$$

Conjecture 7.20 (Integral Novikov conjecture in algebraic K-theory). Assume that $\Gamma$ is torsion free. Then the assembly map

$$
A: H_{*}(B \Gamma ; \mathbb{K}(\mathbb{Z})) \rightarrow K_{*}(\mathbb{Z}[\Gamma])
$$

is injective.

There is also a rational version of the Novikov conjecture in algebraic $K$ theory, i.e., the induced map $A \otimes \mathbb{Q}: H_{*}(B \Gamma ; \mathbb{K}(\mathbb{Z})) \otimes \mathbb{Q} \rightarrow K_{*}(\mathbb{Z}[\Gamma]) \otimes \mathbb{Q}$ is injective.

One fruitful approach to prove the Novikov conjecture for a group $\Gamma$ is to show that the asymptotic dimension of $\Gamma$ is finite.

Theorem 7.21. If a finitely generated group $\Gamma$ has finite asymptotic dimension, asdim $\Gamma<\infty$, and has finite $B \Gamma$, i.e., if its classifying space $B \Gamma$ can be realized as a finite $C W$-complex, then the integral Novikov conjectures in $K$-theory and L-theory hold for $\Gamma$.

We note that the existence of a finite model of $B \Gamma$ implies that $\Gamma$ is torsionfree. For groups containing torsion elements, there is also a modified version of the integral Novikov conjecture. See [92]. 
In [14], the following result was proved.

Proposition 7.22. The asymptotic dimension of $\operatorname{Mod}_{g, n}$ is finite.

For any finite index torsion-free subgroup $\Gamma$ of $\operatorname{Mod}_{g, n}$, the quotient by $\Gamma$ of the thick part of the Teichmüller space $\mathcal{T}_{g, n}(\varepsilon)$ gives a cofinite model of $B \Gamma$-spaces by [95]. One corollary of the above proposition is the following conclusion.

Corollary 7.23. For any finite index subgroup $\Gamma$ of $\operatorname{Mod}_{g, n}$, the rational Novikov conjecture in L-theory holds for $\Gamma$.

This result was proved earlier in [62] [100]. Even though it was not stated in [14], the above discussion shows that the following stronger corollary also holds.

Proposition 7.24. For any finite index torsion-free subgroup $\Gamma$ of $\operatorname{Mod}_{g, n}$, the integral Novikov conjecture in $L$ - and $K$-theories holds for $\Gamma$.

This shows that the results of [14] imply a stronger version of the Novikov conjecture than what was known previously.

\subsection{Non-hyperbolicity of Weil-Petersson metric of Teichmüller space}

As mentioned before, the Teichmüller space $\mathcal{T}_{g, n}$ admits a $\operatorname{Mod}_{g, n}$-equivariant Kähler metric, the Weil-Petersson metric. This is an incomplete metric with strictly negative sectional curvature. It is known that if any simply connected Riemannian manifold with strictly negative sectional curvature admits a compact quotient, then it is a hyperbolic space in the sense of Gromov. The quotient $\operatorname{Mod}_{g, n} \backslash \mathcal{T}_{g, n}$ is non-compact, and one question raised by Bowditch and others was whether the Weil-Petersson metric is hyperbolic in the sense of Gromov.

This problem was solved in [32], and the hyperbolicity of the curve complex $\mathcal{C}\left(S_{g, n}\right)$ was used in the proof.

Proposition 7.25. The Weil-Petersson metric on $\mathcal{T}_{g, n}$ is Gromov-hyperbolic if and only if $3 g-3+n \leq 2$.

Besides the curve complex $\mathcal{C}\left(S_{g, n}\right)$, the pants complex and its quasi-isometry with $\mathcal{T}_{g, n}$ endowed with the Weil-Peterson metric was also used crucially in the proof. See [32] for details. 


\subsection{Heegaard splittings and Hempel distance of 3-manifolds}

Another important application of the curve complex $\mathcal{C}\left(S_{g}\right)$ in 3-dimensional topology is the Hempel distance for Heegaard splittings of 3-dimensional manifolds.

A handlebody is a submanifold with boundary of $\mathbb{R}^{3}$ with only one connected boundary component, which is a compact orientable surface $S_{g}$.

Any closed oriented three dimensional manifold $M^{3}$ can be written as the union of two handlebodies $V_{1}, V_{2}$ of the same genus glued along their boundary,

$$
M=V_{1} \cup_{S_{g}} V_{2}, \quad \partial V_{1} \cong S_{g}, \quad \partial V_{2} \cong S_{g} .
$$

Such a decomposition of $M^{3}$ is called a Heegaard splitting, and $S_{g}$ is called the splitting surface. It is not unique and there are infinitely many different isotropy classes of Heegaard splittings of $M^{3}$.

A simple closed curve in $S_{g}$ is called essential if it does not bound a disc. The identification of $S_{g}$ with the boundary of a handlebody $V_{1}$ is determined by a maximal set $\Delta_{1}$ of disjoint non-homotopic essential simple closed curves in $S_{g}$ that bound essential discs in the handlebody $V_{1}$ (these curves are called meridians). Let $\Delta_{2}$ be a corresponding set for the identification of $S_{g}$ as the boundary of $V_{2}$. The pair $\left(\Delta_{1}, \Delta_{2}\right)$ is called a Heegaard diagram and corresponds to a pair of simplices in $\mathcal{C}\left(S_{g}\right)$.

For each Heegaard splitting $V_{1} \cup_{S_{g}} V_{2}$ with $S_{g}$ as the splitting surface, the set of all possible simplices $\Delta_{1}$ and their faces form a subcomplex $K_{1}$ of $\mathcal{C}\left(S_{g}\right)$ (the subcomplex spanned by the vertices corresponding to all essential simple closed curves of $S_{g}$ that bound some meridians of the handlebody $V_{1}$ ). Similarly, there is a subcomplex $K_{2}$ of $\mathcal{C}\left(S_{g}\right)$ for the other handlebody $V_{2}$.

The Hempel distance for the Heegaard splitting $V_{1} \cup_{S_{g}} V_{2}$ of $M^{3}$ is the distance $d\left(K_{1}, K_{2}\right)$ between $K_{1}$ and $K_{2}$ with respect to the geodesic length function of $\mathcal{C}\left(S_{g}\right)$.

This defines a numerical invariant of the Heegaard splitting of $M$ and is closely related to topological properties of the manifold $M$ and of the Heegaard splitting. For detail, see [77]. The paper [137] discusses behavior of the Hempel distance under stabilization of splitting.

Remark 7.26. In [96], the curve complex $\mathcal{C}\left(S_{g, n}\right)$ is replaced by the pants complex of $S_{g, n}$ and a similar distance function is introduced. The paper [97] contains some results relating the bridge number of hyperbolic knots and an invariant defined in terms of the distance function on $\mathcal{C}\left(S_{g, n}\right)$. The paper [155] applies the idea of Hempel distance to knots and defines a distance for certain knots in lens spaces.

Remark 7.27. The paper [68] shows that for a closed orientable 3-manifold containing an incompressible surface of genus $g$, any Heegaard splitting has 
Hempel distance at most 2g. See also [158] [157] [112] [168] for related results on the Hempel distance.

Remark 7.28. Based on the Hempel distance, the paper [156] defines a translation distance on open book decompositions of three dimensional manifolds, and relates it to the topological properties of the manifolds.

\subsection{Partial compactifications of Teichmüller spaces and their boundaries}

Recall that $\mathcal{T}_{g, n}$ is the Teichmüller space of marked complex structures on $S_{g, n}$, where a marking is a choice of a set of generators of $S_{g, n}$. Then the mapping class group $\operatorname{Mod}_{g, n}$ acts on $\mathcal{T}_{g, n}$ by changing the markings, and the quotient $\operatorname{Mod}_{g, n} \backslash \mathcal{T}_{g, n}$ is the moduli space $\mathcal{M}_{g, n}$ of Riemann surfaces of genus $g$ with $n$ punctures.

It is known that $\mathcal{T}_{g, n}$ is a complex manifold of complex dimension $3 g-3+n$ and diffeomorphic to $\mathbb{R}^{6 g-6+2 n}$, and that $\operatorname{Mod}_{g, n}$ acts holomorphically and properly on $\mathcal{T}_{g, n}$. In particular, $\mathcal{M}_{g, n}$ has a natural structure of a complex orbifold.

Proposition 7.29. For any torsion-free subgroup $\Gamma \subset \operatorname{Mod}_{g, n}, \Gamma \backslash \mathcal{T}_{g, n}$ is a BГ-space.

Proof. Since $\Gamma$ acts properly on $\mathcal{T}_{g, n}$ and is torsion-free, $\Gamma$ acts fixed-point freely on $\mathcal{T}_{g, n}$. Since $\mathcal{T}_{g, n}$ is contractible, it is an $E \Gamma$-space, and $\Gamma \backslash \mathcal{T}_{g, n}$ is a $B \Gamma$-space.

A more general result is true for subgroups of $\operatorname{Mod}_{g, n}$ containing torsion elements.

Proposition 7.30. For any subgroup $\Gamma \subset \operatorname{Mod}_{g, n}, \mathcal{T}_{g, n}$ is an $\underline{E} \Gamma$-space.

We only need to check that for any finite subgroup $F \subset \Gamma$, the set of fixed points $\mathcal{T}_{g, n}^{F}$ is nonempty and contractible. The former follows from the solution of the Nielsen realization problem, and the latter from either the existence of a unique left (or right) earthquake between any two points or the convexity of the Weil-Petersson metric. See [95] for more details.

It is known that $\mathcal{M}_{g, n}=\operatorname{Mod}_{g, n} \backslash \mathcal{T}_{g, n}$ is noncompact. Therefore, $\mathcal{T}_{g, n}$ is not a cofinite $\underline{E} \operatorname{Mod}_{g, n}$-space. To see this, we assume for simplicity that $2 g-2+n>$ 0 . Then every Riemann surface in $\mathcal{M}_{g, n}$ admits a unique hyperbolic metric of finite area which is conformal to the complex structure. By pinching a simple closed geodesic on a hyperbolic surface $\Sigma_{g, n}$, we obtain a family of Riemann surfaces in $\mathcal{M}_{g, n}$ that has no limit. 
For a non-uniform torsion-free arithmetic subgroup of a linear semisimple algebraic group $\mathbf{G}, \Gamma \backslash X$ is a non-cofinite $B \Gamma$-space. To obtain a cofinite $B \Gamma$ space, this problem was solved by the Borel-Serre compactification of $\Gamma \backslash X$, which is a quotient of the Borel-Serre partial compactification of the associated symmetric space $X=G / K$.

In order to construct an analogue of the Borel-Serre partial compactification of $\mathcal{T}_{g, n}$, Harvey [69] [70] [73] introduced the curve complex $\mathcal{C}\left(S_{g, n}\right)$.

To explain the relation between partial compactifications of $\mathcal{T}_{g, n}$ and simplices of $\mathcal{C}\left(S_{g, n}\right)$, we note the following compactness criterion of Mumford.

Proposition 7.31. A sequence of Riemann surfaces $\Sigma_{j}$ in $\mathcal{M}_{g, n}$ has no accumulation point in $\mathcal{M}_{g, n}$ if and only if there is a disjoint collection of simple closed geodesics $\gamma_{j, 1}, \cdots, \gamma_{j, r_{j}}$ with respect to the hyperbolic metric of $\Sigma_{j}$ whose lengths go to 0 as $j \rightarrow+\infty$, i.e., that can be simultaneously pinched.

Note that in the above proposition, the geodesics must be disjoint since the collar theorem for hyperbolic surfaces implies that two sufficiently short geodesics are disjoint and hence simultaneously pinched geodesics must be disjoint.

Conversely, a collection of disjoint simple closed geodesics in a hyperbolic surface $\Sigma_{g, n}$ can be pinched simultaneously if and only if they are disjoint. This can be seen from pants decompositions of hyperbolic surfaces.

Given the above discussion, a natural compactification of $\mathcal{M}_{g, n}$ is obtained by adding degenerate Riemann surfaces. This is the Deligne-Mumford compactification $\overline{\mathcal{M}}_{g, n}{ }^{D M}$ of $\mathcal{M}_{g, n}$.

As a topological space, $\overline{\mathcal{M}}_{g, n} D M$ can be constructed as a quotient of the augmented Teichmüller space $\hat{\mathcal{T}}_{g, n}$. For every simplex $\sigma$ of $\mathcal{C}\left(S_{g, n}\right)$, there is a boundary Teichmüller space $\mathcal{T}_{g, n ; \sigma}$, which is the Teichmüller space of the surface obtained from $S_{g, n}$ by cutting along the curves in $\sigma$. In terms of the hyperbolic metric, every homotopy class of curves in $\sigma$ determines a unique simple closed geodesic in every marked hyperbolic surface in $\mathcal{T}_{g, n}$, and degenerated hyperbolic surfaces in the boundary $\mathcal{T}_{g, n ; \sigma}$ are obtained by pinching exactly the geodesics in $\sigma$. Then the augmented Teichmüller space $\hat{\mathcal{T}}_{g, n}$ is

$$
\hat{\mathcal{T}}_{g, n}=\mathcal{T}_{g, n} \cup \coprod_{\sigma \in \mathcal{C}\left(S_{g, n}\right)} \mathcal{T}_{g, n ; \sigma}
$$

The action of $\operatorname{Mod}_{g, n}$ on $\mathcal{T}_{g, n}$ extends to a continuous action on $\hat{\mathcal{T}}_{g, n}$ and the quotient $\operatorname{Mod}_{g, n} \backslash \hat{\mathcal{T}}_{g, n}$ is homeomorphic to $\overline{\mathcal{M}}_{g, n}{ }^{D M}$.

An important point here is that the curve complex $\mathcal{C}\left(S_{g, n}\right)$ is a parameter space for the boundary components of the augmented Teichmüller space $\hat{\mathcal{T}}_{g, n}$ [69] [174]. Since each boundary Teichmüller space $\mathcal{T}_{g, n ; \sigma}$ is contractible, the 
boundary $\partial \hat{\mathcal{T}}_{g, n}$ is homotopy equivalent to $\mathcal{C}\left(S_{g, n}\right)$ and hence is homotopy equivalent to a bouquet of spheres.

Proposition 7.32. The action of $\operatorname{Mod}_{g, n}$ on $\hat{\mathcal{T}}_{g, n}$ is not proper. For every point in a boundary Teichmüller space $\mathcal{T}_{g, n ; \sigma}$, its stabilizer in $\operatorname{Mod}_{g, n}$ contains an infinite subgroup, which is generated by the Dehn twists along the curves in $\sigma$ and is isomorphic to $\mathbb{Z}^{\sigma}$, as a subgroup of finite index.

This implies that for any finite-index subgroup $\Gamma \subset \operatorname{Mod}_{g, n}$, the inclusion $\Gamma \backslash \mathcal{T}_{g, n} \rightarrow \Gamma \backslash \hat{\mathcal{T}}_{g, n}$ is not a homotopy equivalence. This is similar to the situation with the reductive Borel-Serre compactification of $\Gamma \backslash X$ in $\S 6.3$.

To overcome the problem of infinite stabilizers $\mathbb{Z}^{\sigma}$ for points in $\mathcal{T}_{g, n ; \sigma}$, we need to enlarge the boundary component to $\mathbb{R}^{\sigma} \times \mathcal{T}_{g, n ; \sigma}$. The resulting space is a Borel-Serre partial compactification $\overline{\mathcal{T}}_{g, n}{ }^{B S}$ of $\mathcal{T}_{g, n}$ constructed in [70] [73] [82]:

$$
{\overline{\mathcal{T}_{g, n}}}^{B S}=\mathcal{T}_{g, n} \cup \coprod_{\sigma \in \mathcal{C}\left(S_{g, n}\right)} \mathbb{R}^{\sigma} \times \mathcal{T}_{g, n ; \sigma}
$$

Proposition 7.33. The action of $\operatorname{Mod}_{g, n}$ on $\mathcal{T}_{g, n}$ extends to a continuous and proper action on $\overline{\mathcal{T}}_{g, n} B S$ with a compact quotient $\operatorname{Mod}_{g, n} \backslash \overline{\mathcal{T}}_{g, n} B S$. The boundary of the Borel-Serre partial compactification $\overline{\mathcal{T}_{g, n}} B S$ is homotopy equivariant to $\mathcal{C}\left(S_{g, n}\right)$ and hence to a bouquet of spheres $S^{d}$, where $d=2 g-2=-\chi\left(S_{g}\right)$ if $n=0, d=-\chi\left(S_{g, n}\right)-1=2 g-3+n$ if $g \geq 1$ and $n>0$, and $d=-\chi\left(S_{0, n}\right)-2=n-4$ if $g=0$.

Corollary 7.34. For any finite-index torsion-free subgroup $\Gamma \subset \operatorname{Mod}_{g, n}$, $\overline{\mathcal{T}}_{g, n}{ }^{B S}$ is a cofinite $\underline{E} \Gamma$-space and the quotient $\Gamma \backslash \overline{\mathcal{T}}_{g, n} B S$ is a finite BГ-space.

Proof. Since $\overline{\mathcal{T}_{g, n}}{ }^{B S}$ is a manifold with corners whose interior is equal to $\mathcal{T}_{g, n}$, it is contractible. Since $\Gamma$ is torsion-free, it acts fixed-point freely on $\overline{\mathcal{T}}_{g, n}{ }^{B S}$, and the quotient $\Gamma \subset \operatorname{Mod}_{g, n}$ is a compact manifold with corners. Therefore, the quotient $\Gamma \backslash \overline{\mathcal{T}}_{g, n} B S$ is a finite $B \Gamma$-space.

On the other hand, for $\operatorname{Mod}_{g, n}$ or its finite index subgroups $\Gamma$ which contain torsion elements, it is not clear whether $\overline{\mathcal{T}}_{g, n} B S$ is an $\underline{E} \Gamma$-space.

To solve this problem, we can take suitable subspaces of $\mathcal{T}_{g, n}$ instead of a compactification. For any sufficiently small $\varepsilon>0$, define the $\varepsilon$-thick part

$$
\mathcal{T}_{g, n}(\varepsilon)=\left\{\Sigma \in \mathcal{T}_{g, n} \mid \text { for any simple closed geodesic } \gamma \subset \Sigma, \ell(\gamma) \geq \varepsilon\right\},
$$

where $\ell(\gamma)$ is the length of the geodesic $\gamma$. 
Proposition 7.35. The thick part $\mathcal{T}_{g, n}(\varepsilon)$ is a submanifold with corners and invariant under the action of $\operatorname{Mod}_{g, n}$ with a compact quotient. The boundary faces of $\mathcal{T}_{g, n}(\varepsilon)$ are also parametrized by the simplices of $\mathcal{C}\left(S_{g, n}\right)$ and contractible, and the boundary $\partial \mathcal{T}_{g, n}(\varepsilon)$ is homotopy equivalent to a wedge of spheres $S^{d}$, where $d=2 g-2=-\chi\left(S_{g}\right)$ if $n=0, d=-\chi\left(S_{g, n}\right)-1=2 g-3+n$ if $g \geq 1$ and $n>0$, and $d=-\chi\left(S_{0, n}\right)-2=n-4$ if $g=0$.

In [95], we proved

Proposition 7.36. There is a $\operatorname{Mod}_{g, n}$-equivariant deformation retraction of $\mathcal{T}_{g, n}$ to $\mathcal{T}_{g, n}(\varepsilon)$, and hence $\mathcal{T}_{g, n}(\varepsilon)$ is a cofinite $\underline{E} \operatorname{Mod}_{g, n}$-space.

If $n>0$, there is another equivariant deformation of $\mathcal{T}_{g, n}$ which is of the smallest possible dimension, i.e., the virtual cohomological dimension of $\operatorname{Mod}_{g, n}$ in [66].

Problem 7.37. When $n=0$, construct a $\operatorname{Mod}_{g, 0}$-deformation retract of $\mathcal{T}_{g, 0}$ of dimension equal to the virtual cohomological dimension of $\operatorname{Mod}_{g}$, which is equal to $4 g-5$.

This problem seems to be completely open. It is not clear what subspaces of $\mathcal{T}_{g, n}$ are possible candidates for such a retract. It is not abvious if such a retract exists.

\subsection{Cohomological dimension and duality properties of mapping class groups}

As discussed earlier, one important application of the Borel-Serre partial compactification of a symmetric space concerns the virtual cohomological dimension and duality properties of arithmetic subgroups. The analogous partial compactifications of $\mathcal{T}_{g, n}$ in the previous subsection have similar applications.

Proposition 7.38. When the Euler characteristic $\chi\left(S_{g, n}\right)$ is positive, $\operatorname{Mod}_{g, n}$ is a virtual duality group of dimension $d$, where $d=4 g-5=\operatorname{dim} \mathcal{T}_{g}+\chi\left(S_{g}\right)-1$ if $n=0$, and $d=4 g-4+n=\operatorname{dim} \mathcal{T}_{g, n}+\chi\left(S_{g, n}\right)$ if $g \geq 1$ and $n>0$, and $d=n-3=\operatorname{dim} \mathcal{T}_{0, n}+\chi\left(S_{0, n}\right)+1$. The dualizing module is equal to the only nonzero reduced homology group of $\mathcal{C}\left(S_{g, n}\right)$, called the Steinberg module of $\operatorname{Mod}_{g, n}$.

The proof is similar to the proof for arithmetic groups using results on the Borel-Serre partial compactification of $\mathcal{T}_{g, n}$ in the previous subsection. See [66] [67] [80] [69] for details.

In [86], using Proposition 5.9 on the topology of $\mathcal{C}\left(S_{g, n}\right)$, we proved 
Proposition 7.39. $\operatorname{Mod}_{g, n}$ is not a virtual Poincaré duality group.

This proposition is equivalent to the fact that when $\operatorname{dim} \mathcal{T}_{g, n}>0$, the Steinberg module of $\operatorname{Mod}_{g, n}$ is of infinite rank. See [31] for more information on this module.

Since $\operatorname{Mod}_{g, n} \backslash \mathcal{T}_{g, n}$ is noncompact, Proposition 7.39 is consistent with the result that an arithmetic subgroup $\Gamma$ is not a virtual Poincaré duality group if and only if $\Gamma \backslash X$ is noncompact (Proposition 6.14).

The Euler characteristic of $\operatorname{Mod}_{g, n}$ is given by special values of the Riemann zeta function, and the homology groups of $\operatorname{Mod}_{g, n}$ in low degrees are also known. See [66] and references there.

Problem 7.40. Compute $H_{i}\left(\operatorname{Mod}_{g, n}, \mathbb{Z}\right)$ in all degrees. Maybe compute them for special values of $g$ and $n$, and also for special values of $i$ first.

\subsection{Tangent cones at infinity of Teichmüller spaces, moduli spaces, and mapping class groups}

As discussed earlier, the asymptotic cones at infinity of symmetric spaces and locally symmetric spaces are given by buildings. A natural problem is to consider the asymptotic cones of the Teichmüller space $\mathcal{T}_{g, n}$ and its quotient $\mathcal{M}_{g, n}=\operatorname{Mod}_{g, n} \backslash \mathcal{T}_{g, n}$.

Proposition 7.41. With respect to the Teichmüller metric, for any finite index subgroup $\Gamma \subset \operatorname{Mod}_{g, n}$, the asymptotic cone at infinity $\mathrm{Cone}_{\infty}\left(\Gamma \backslash \mathcal{T}_{g, n}\right)$ is equal to the metric cone over $\Gamma \backslash \mathcal{C}\left(S_{g, n}\right)$.

This result was proved in [111] [53]. There are some results on $\mathrm{Cone}_{\infty}\left(\operatorname{Mod}_{g, n}\right)$ and $\mathrm{Cone}_{\infty}\left(\mathcal{T}_{g, n}, d_{W P}\right)$, where $d_{W P}$ is the Weil-Petersson metric.

By [5], for any asymptotic cone $\mathrm{Cone}_{\infty}\left(\operatorname{Mod}_{g, n}\right)$, every point is a global cut-point, i.e., Cone ${ }_{\infty}\left(\operatorname{Mod}_{g, n}\right)$ is tree-graded.

Since the quotient $\operatorname{Mod}_{g, n} \backslash \mathcal{T}_{g, n}$ is noncompact, it is reasonable to expect that $\operatorname{Mod}_{g, n}$ is not quasi-isometric to $\mathcal{T}_{g, n}$, and that $\operatorname{Cone}_{\infty}\left(\mathcal{T}_{g, n}\right)$ and Cone $_{\infty}\left(\operatorname{Mod}_{g, n}\right)$ will be different. The following bounds on the dimension of Cone $_{\infty}\left(\operatorname{Mod}_{g, n}\right)$ and $\mathrm{Cone}_{\infty}\left(\mathcal{T}_{g, n}, d_{W P}\right)$ were obtained in [7].

Proposition 7.42. The maximal dimension of locally compact subsets of theasymptoticcone $\mathrm{Cone}_{\infty}\left(\operatorname{Mod}_{g, n}\right)$ is equal to the maximal rank of free abelian subgroups of $\operatorname{Mod}_{g, n}$, which is equal to $3 g-3+n$. Consequently, the geomet-

ric rank of $\operatorname{Mod}_{g, n}$ is equal to the maximal rank of free abelian subgroups of $\operatorname{Mod}_{g, n}$. 
The geometric rank of a group $\Gamma$ is defined as the largest integer $n$ for which there exists a quasi-isometric embedding $\mathbb{Z}^{n} \rightarrow \Gamma$. This result was used to prove the quasi-isometric rigidity of $\operatorname{Mod}_{g, n}$ in [6] [65]. See $\S 7.4$.

Proposition 7.43. The maximal dimension of locally compact subsets of the asymptotic cone $\mathrm{Cone}_{\infty}\left(\mathcal{T}_{g, n}, d_{W P}\right)$ is equal to $\left[\frac{3 g+p-2}{2}\right]$, that is, the integral part of $\frac{3 g+p-2}{2}$, and hence the geometric rank of $\left(\mathcal{T}_{g, n}, d_{W P}\right)$ is equal to $\left[\frac{3 g+p-2}{2}\right]$.

The geometric rank of a metric space is the maximal dimension of quasiflats in the metric space. The geometric ranks of some complexes related to the curve complex were determined in [136].

Problem 7.44. Understand the global structure of the asymptotic cones at infinity, Cone $e_{\infty}\left(\operatorname{Mod}_{g, n}\right)$ and $C o n e_{\infty}\left(\mathcal{T}_{g, n}, d_{W P}\right)$, and determine the maximal dimension of compact subsets of the asymptotic cone at infinity Cone $_{\infty}\left(\mathcal{T}_{g, n}, d_{T e i}\right)$, where $d_{T e i}$ is the Teichmüller metric.

There are some other results that connect the geometry and topology of $\mathcal{M}_{g, n}$ at infinity with the curve complex $\mathcal{C}\left(S_{g, n}\right)$.

Proposition 7.45. Assume that $\operatorname{dim} \mathcal{T}_{g, n} \geq 2$. Then for any finite index subgroup $\Gamma \subset \operatorname{Mod}_{g, n}$, the quotient $\Gamma \backslash \mathcal{T}_{g, n}$ has only one end.

Briefly, the reason is that under the assumption that $\operatorname{dim} \mathcal{T}_{g, n} \geq 2, S_{g, n}$ contains at least two simple closed essential curves, and $\mathcal{C}\left(S_{g, n}\right)$ is connected. Now a suitable neighborhood of the infinity of $\Gamma \backslash \mathcal{T}_{g, n}$ is homotopy equivalent to $\Gamma \backslash \mathcal{C}\left(S_{g, n}\right)$ and hence is connected.

Proposition 7.46. For any finite-index subgroup $\Gamma \subset \operatorname{Mod}_{g, n}$, the quotient $\Gamma \backslash \mathcal{T}_{g, n}$ has a compactification $\Gamma \backslash \mathcal{T}_{g, n} \cup \Gamma \backslash \mathcal{C}\left(S_{g, n}\right)$ whose boundary is equal to the finite complex $\Gamma \backslash \mathcal{C}\left(S_{g, n}\right)$.

This is similar to the Tits compactification of locally symmetric spaces in [94]. See [90, Proposition 12.6].

\subsection{Simplicial volumes of moduli spaces}

As recalled in $\S 6.5$, for an arithmetic locally symmetric space $\Gamma \backslash X$, whether its simplicial volume vanishes or not depends on the size of its $\mathbb{Q}$-rank, which is related to the dimension of the spheres that determine the homotopy type of the Tits building $\Delta_{\mathbb{Q}}(\mathbf{G})$. It turns out that similar results hold for $\mathcal{M}_{g, n}$ [91]. 
Proposition 7.47. The orbifold simplicial volume of $\mathcal{M}_{g}$ is equal to zero if and only if $g \geq 2$. The simplicial volume of $\mathcal{M}_{g}$ vanishes for all $g \geq 1$.

It is known that $\operatorname{Mod}_{g, n}$ admits finite index torsion-free subgroups and hence $\mathcal{M}_{g, n}$ admits finite smooth covers. Since the orbifold simplicial volume is greater than or equal to the simplicial volume [91], it suffices to show that the orbifold simplicial volume of $\mathcal{M}_{g}$ is equal to zero if and only if $g \geq 2$, and the simplicial volume of $\mathcal{M}_{1}$ is equal to 0 .

For the first statement, the proof is similar to the proof of Proposition 6.17 by replacing the Borel-Serre partial compactification of the symmetric space by the thick part $\mathcal{T}_{g}(\varepsilon)$ of the Teichmüller space $\mathcal{T}_{g}$, and the rational Tits building $\Delta_{\mathbb{Q}}(\mathbf{G})$ by the curve complex $\mathcal{C}\left(S_{g}\right)$. Since $\mathcal{C}\left(S_{g}\right)$ is homotopy equivalent to a bouquet of spheres of dimension $2 g-2$ for $g \geq 2$, it is simply connected. This is a crucial ingredient of the proof of the above proposition. The nonvanishing of the orbifold simplicial volume of $\mathcal{M}_{1} \cong \mathrm{SL}(2, \mathbb{Z}) \backslash \mathbf{H}^{2}$ follows from the result of Thurston for finite volume hyperbolic manifolds (Proposition 6.16).

To prove that the simplicial volume of $\mathrm{SL}(2, \mathbb{Z}) \backslash \mathbf{H}^{2}$ vanishes, we note that $\mathrm{SL}(2, \mathbb{Z}) \backslash \mathbf{H}^{2}$ is homeomorphic to $\mathbb{R}^{2}$. Since the orbifold simplicial volume and hence the simplicial volume of $\mathrm{SL}(2, \mathbb{Z}) \backslash \mathbf{H}^{2}$ is finite, this implies that the simplicial volume of $\mathbb{R}^{2}$ is finite. Since $\mathbb{R}^{2}$ admits proper self-maps of degree greater than 2 , its simplicial volume is 0 . Hence the simplicial volume of $\mathrm{SL}(2, \mathbb{Z}) \backslash \mathbf{H}^{2}$ is 0 . This also gives a new proof of the fact that the simplicial volume of $\mathbb{R}^{2 n}$ vanishes. See [91] for details.

More generally, for the general moduli space $\mathcal{M}_{g, n}$, we have

Proposition 7.48. When $g \geq 1$ and $n>0$, both the simplicial volume and orbifold simplicial volume of $\mathcal{M}_{g, n}$ are equal to zero if $-\chi\left(S_{g, n}\right)-1 \geq 2$, i.e., $2 g+n \geq 5$; and when $g=0$ and $n \geq 4$, the simplicial volume of $\mathcal{M}_{0, n}$ if $-\chi\left(S_{0, n}\right)-2 \geq 2$, i.e., $n \geq 6$.

It is known that $\mathcal{M}_{1,1} \cong \mathcal{M}_{1,0}=\operatorname{SL}(2, \mathbb{Z}) \backslash \mathbf{H}^{2}$, which is homeomorphic to $\mathbb{R}^{2}$. Since the simplicial volume of $\mathbb{R}^{2}$ is equal to 0 , the simplicial volume of $\mathcal{M}_{1,1}$ and $\mathcal{M}_{1,0}$ is also equal to 0 . On the other hand, by Proposition 6.16, any finite smooth cover of $\mathcal{M}_{1,1}$ and $\mathcal{M}_{1,0}$ have positive simplicial volume. Therefore, the orbifold simplicial volume of $\mathcal{M}_{1,1}$ and $\mathcal{M}_{1,0}$ is positive.

Since $\mathcal{T}_{0,4} \cong \mathcal{T}_{1,1} \cong \mathbf{H}^{2}[142]$ and $\operatorname{Mod}_{0,4}$ is commensurable with $\operatorname{SL}(2, \mathbb{Z})$, it follows from that for $(g, n)=(1,0),(1,1),(0,4)$, the orbifold simplicial volume of $\mathcal{M}_{g, n}$ is positive.

Problem 7.49. Decide whether the simplicial volume and orbifold simplicial volume of $\mathcal{M}_{g, n}$ vanish or not for $(g, n)=(1,2),(0,5)$. 


\subsection{The action of $\operatorname{Mod}_{g, n}$ on $\mathcal{C}\left(S_{g, n}\right)$ and applications}

As mentioned before, $\operatorname{Mod}_{g, n}$ acts on $\mathcal{C}\left(S_{g, n}\right)$, and this action can be used to understand structures and representations of $\operatorname{Mod}_{g, n}$.

One of the early applications of the curve complex by Harvey [71] concerns a simplified proof of the classification of elements of $\operatorname{Mod}_{g, n}$ by Thurston [164].

Recall that the group $\mathrm{SL}(2, \mathbb{R})$ acts on $\mathbf{H}^{2}$ by Mobius transformations and that any element $\gamma \in \mathrm{SL}(2, \mathbb{R})$ belongs to one of the following types:

(1) $\gamma$ is elliptic and is characterized by the property that it fixes a point in $\mathbf{H}^{2}$

(2) $\gamma$ is parabolic and is characterized by the property that it fixes exactly one point in the boundary $\mathbf{H}^{2}(\infty) \cong S^{1}$;

(3) $\gamma$ is hyperbolic and is characterized by the property that it fixes exactly two points in the boundary $\mathbf{H}^{2}(\infty) \cong S^{1}$.

Since $\mathbf{H}^{2} \cup \mathbf{H}^{2}(\infty)$ is a closed ball and $\gamma$ acts on it continuously, it must fix some point in $\mathbf{H}^{2} \cup \mathbf{H}^{2}(\infty)$, and the three possibilities above cover all cases.

For any discrete subgroup $\Gamma \subset \operatorname{SL}(2, \mathbb{R})$, any element $\gamma \in \Gamma$ belongs to one of the following types:

(1) $\gamma$ is periodic, i.e., there exists some integer $n$ such that $\gamma^{n}=I d$;

(2) $\gamma$ is parabolic and fixes a boundary point in $\mathbf{H}^{2}(\infty)$;

(3) $\gamma$ is hyperbolic and fixes exactly two points in the boundary $\mathbf{H}^{2}(\infty) \cong$ $S^{1}$.

Thurston [164] defined a compactification ${\overline{\mathcal{T}_{g, n}}}^{T h}$ of $\mathcal{T}_{g, n}$ through projective measured laminations such that

(1) $\overline{\mathcal{T}}_{g, n}{ }^{T h}$ is homeomorphic to a closed ball of dimension $6 g-6+n$;

(2) the action of $\operatorname{Mod}_{g, n}$ extends to a continuous action on ${\overline{\mathcal{T}_{g, n}}}^{T h}$.

Using this action, he classified elements $\gamma \in \operatorname{Mod}_{g, n}$ into corresponding three types:

(1) $\gamma$ is periodic, i.e., there exists some integer $n$ such that $\gamma^{n}=I d$, and is characterized by the condition that $\gamma$ fixes a point in $\mathcal{T}_{g, n}$;

(2) $\gamma$ is reducible, i.e., $\gamma$ leaves invariant a collection of disjoint simple closed essential curves of $S_{g, n}$;

(3) $\gamma$ is pseudo-Anosov and is characterized by the condition that $\gamma$ fixes exactly two points in the boundary $\partial{\overline{\mathcal{T}_{g, n}}}^{T h}$.

Remark 7.50. The action of $\operatorname{Mod}_{g, n}$ on $\mathcal{C}\left(S_{g, n}\right)$ can be used to define a translation length of an element $\gamma$ of $\operatorname{Mod}_{g, n}$ : for any simple closed curve $\alpha$ in $S_{g, n}$, the translation length of $\gamma$ is defined by

$$
\liminf _{j \rightarrow+\infty} \frac{d_{\mathcal{C}}\left(\alpha, f^{j}(\alpha)\right)}{j}
$$


where $d_{\mathcal{C}}\left(\alpha, f^{j}(\alpha)\right)$ is the distance between the two vertices $\alpha$ and $f^{j}(\alpha)$ of $\mathcal{C}\left(S_{g, n}\right)$. It was proved in [125] that an element $\gamma$ is pseudo-Anosov if and only if its translation length is positive. See [52] [56] for some estimates on the translation length.

Remark 7.51. An important application of the action of $\operatorname{Mod}_{g, n}$ on the curve complex $\mathcal{C}\left(S_{g, n}\right)$ is to give an explicit presentation of $\operatorname{Mod}_{g, n}$ in [78] [169] (see also [72]). Another presentation was constructed via the action of $\operatorname{Mod}_{g, n}$ on a variant of the curve complex in [11].

Remark 7.52. The action of $\operatorname{Mod}_{g, n}$ on the curve complex $\mathcal{C}\left(S_{g, n}\right)$ is also used essentially in the proof of the Tits alternative for $\operatorname{Mod}_{g, n}$ [16] [85].

Remark 7.53. The action of $\operatorname{Mod}_{g, n}$ on the pants complex [74] was also used to construct a presentation of $\operatorname{Mod}_{g, n}$.

Remark 7.54. Another application of the action of $\operatorname{Mod}_{g, n}$ on $\mathcal{C}\left(S_{g, n}\right)$ is to construct irreducible unitary representations of $\operatorname{Mod}_{g, n}$. It was shown in [141] that the action of $\operatorname{Mod}_{g, n}$ on $\mathcal{C}\left(S_{g, n}\right)$ has non-commensurable stabilizers and a general method was used to produce irreducible unitary representations.

Remark 7.55. By [125], there is a relative metric on $\operatorname{Mod}_{g, n}$, which is quasiisometric to $\mathcal{C}\left(S_{g, n}\right)$. (A relative metric on a group is a word metric on the group with respect to an infinite generating set, consisting of a finite generating set and a finite collection of subgroups.) The paper [118] shows that a random walk on $\operatorname{Mod}_{g, n}$ makes linear progress in this relative metric.

\section{References}

[1] P. Abramenko, Twin buildings and applications to $S$-arithmetic groups, Lecture Notes in Mathematics, 1641. Springer-Verlag, Berlin, 1996. x+123 pp.

[2] P. Abramenko, K. Brown, Buildings, Theory and applications, Graduate Texts in Mathematics, 248. Springer, New York, 2008. xxii+747 pp.

[3] W. Ballmann, Nonpositively curved manifolds of higher rank, Ann. of Math. (2) 122 (1985), no. 3, 597-609.

[4] W. Ballmann, M. Gromov, V. Schroeder, Manifolds of nonpositive curvature, Progress in Mathematics, 61. Birkhäuser Boston, Inc., Boston, MA, 1985. $\mathrm{vi}+263 \mathrm{pp}$.

[5] J. Behrstock, Asymptotic geometry of the mapping class group and Teichmüller space, Geom. Topol. 10 (2006), 1523-1578. 
[6] J. Behrstock, B. Kleiner, Y. Minsky, L. Mosher, Geometry and rigidity of mapping class groups, arXiv:0801.2006.

[7] J. Behrstock, Y. Minsky, Dimension and rank for mapping class groups, Ann. of Math. (2) 167 (2008), no. 3, 1055-1077.

[8] G. Bell, K. Fujiwara, The asymptotic dimension of a curve graph is finite, J. Lond. Math. Soc. (2) 77 (2008), no. 1, 33-50.

[9] R. Bell, D. Margalit, Injections of Artin groups, Comment. Math. Helv. 82 (2007), no. 4, 725-751.

[10] R. Benedetti, C. Petronio, Lectures on hyperbolic geometry, Universitext. Springer-Verlag, Berlin, 1992. xiv+330 pp.

[11] S. Benvenuti, Finite presentations for the mapping class group via the ordered complex of curves, Adv. Geom. 1 (2001), no. 3, 291-321.

[12] L. Bers, The moduli of Kleinian groups. Russian Math. Surveys 29 (1974), no. $2,88-102$.

[13] G. Besson, G. Courtois, S. Gallot, Entropies et rigidités des espaces localement symétriques de courbure strictement négative, Geom. Funct. Anal. 5 (1995), no. 5, 731-799.

[14] M. Bestvina, K. Bromberg, K. Fujiwara, The asymptotic dimension of mapping class groups is finite, arXiv:1006.1939.

[15] M. Bestvina, K. Fujiwara, Bounded cohomology of subgroups of mapping class groups, Geom. Topol. 6 (2002), 69-89

[16] J. Birman, A. Lubotzky, J. McCarthy, Abelian and solvable subgroups of the mapping class groups. Duke Math. J. 50 (1983), no. 4, 1107-1120.

[17] M. Boggi, P. Lochak Profinite complexes of curves, their automorphisms and anabelian properties of moduli stacks of curves, arXiv:0706.0859.

[18] F. Bonahon, Bouts des variétés hyperboliques de dimension 3. Ann. of Math. (2) 124 (1986), no. 1, 71-158.

[19] A. Borel, L. Ji, Compactifications of symmetric and locally symmetric spaces, Mathematics: Theory \& Applications. Birkhäuser Boston, Inc., Boston, MA, 2006. xvi+479 pp.

[20] A. Borel, J. P. Serre, Corners and arithmetic groups, Comment. Math. Helv. 48 (1973), 436-491.

[21] A. Borel, J. P. Serre, Cohomologie d'immeubles et de groupes Sarithmétiques, Topology 15 (1976), no. 3, 211-232.

[22] M. Bourdon, Sur les immeubles fuchsiens et leur type de quasi-isométrie, Ergodic Theory Dynam. Systems 20 (2000), no. 2, 343-364.

[23] B. Bowditch, Intersection numbers and the hyperbolicity of the curve complex, J. Reine Angew. Math. 598 (2006), 105-129.

[24] B. Bowditch, Tight geodesics in the curve complex, Invent. Math. 171 (2008), no. 2, 281-300. 
[25] B. Bowditch, Length bounds on curves arising from tight geodesics, Geom. Funct. Anal. 17 (2007), no. 4, 1001-1042.

[26] B. Bowditch, Geometrical finiteness for hyperbolic groups, J. Funct. Anal. 113 (1993), no. 2, 245-317.

[27] B. Bowditch, Geometric models for hyperbolic 3-manifolds, preprint.

[28] B. Bowditch, End invariants of hyperbolic 3-manifolds, preprint.

[29] M. Bridson, Geodesics and curvature in metric simplicial complexes, in Group theory from a geometrical viewpoint, 373-463, World Sci. Publ., River Edge, NJ, 1991.

[30] M. Bridson, A. Haefliger, Metric spaces of non-positive curvature, Grundlehren der Mathematischen Wissenschaften, 319. Springer-Verlag, Berlin, 1999. xxii+643 pp.

[31] N. Broaddus, Homology of the curve complex and the Steinberg module of the mapping class group, arXiv:0711.0011.

[32] J. Brock, B. Farb, Curvature and rank of Teichmüller space. Amer. J. Math. 128 (2006), no. 1, 1-22.

[33] J. Brock, The Weil-Petersson metric and volumes of 3-dimensional hyperbolic convex cores, J. Amer. Math. Soc. 16 (2003), no. 3, 495-535

[34] J. Brock, R. Canary, Y. Minsky, The classification of Kleinian surface groups, II: The Ending Lamination Conjecture, arXiv:math/0412006v1.

[35] J. Brock, H. Masur, Y. Minsky, Asymptotics of Weil-Petersson geodesic. I. Ending laminations, recurrence, and flows. Geom. Funct. Anal. 19 (2010), no. $5,1229-1257$.

[36] K. Brown, Cohomology of groups, Corrected reprint of the 1982 original. Graduate Texts in Mathematics, 87. Springer-Verlag, New York, 1994. $\mathrm{x}+306 \mathrm{pp}$.

[37] M. Bucher-Karlsson, Simplicial volume of locally symmetric spaces covered by $\mathrm{SL}_{3} \mathbb{R} / \mathrm{SO}(3)$. Geom. Dedicata 125 (2007), 203-224.

[38] K. Burns, R. Spatzier, Manifolds of nonpositive curvature and their buildings, Inst. Hautes Études Sci. Publ. Math. No. 65 (1987), 35-59.

[39] K. Burns, R. Spatzier, On topological Tits buildings and their classification, Inst. Hautes Études Sci. Publ. Math. No. 65 (1987), 5-34.

[40] P. Buser, Geometry and spectra of compact Riemann surfaces, Progress in Mathematics, 106. Birkhäuser Boston, Inc., Boston, MA, 1992. xiv+454 pp.

[41] D. Canary, Ends of hyperbolic 3-manifolds, J. Amer. Math. Soc. 6 (1993), no. $1,1-35$.

[42] D. Canary, Marden's tameness conjecture: history and applications, in Geometry, analysis and topology of discrete groups, 137-162, Adv. Lect. Math. (ALM), 6, Int. Press, Somerville, MA, 2008. 
[43] R. Canary, D. McCullough, Homotopy equivalences of 3-manifolds and deformation theory of Kleinian groups, Mem. Amer. Math. Soc. 172 (2004), no. 812 , xii $+218 \mathrm{pp}$.

[44] R. Carter, Finite groups of Lie type. Conjugacy classes and complex characters, Pure and Applied Mathematics (New York). A Wiley-Interscience Publication. John Wiley \& Sons, Inc., New York, 1985. xii+544 pp.

[45] M. Davis, The geometry and topology of Coxeter groups, London Mathematical Society Monographs Series, 32. Princeton University Press, Princeton, NJ, 2008. xvi+584 pp.

[46] M. Davis, Examples of buildings constructed via covering spaces, Groups Geom. Dyn. 3 (2009), no. 2, 279-298.

[47] A. Eskin, Quasi-isometric rigidity of nonuniform lattices in higher rank symmetric spaces. J. Amer. Math. Soc. 11 (1998), no. 2, 321-361.

[48] A. Eskin, B. Farb, Quasi-flats and rigidity in higher rank symmetric spaces, J. Amer. Math. Soc. 10 (1997), no. 3, 653-692.

[49] J. Essert, Buildings, group homology and lattices, arXiv:1008.4908.

[50] B. Farb, The quasi-isometry classification of lattices in semisimple Lie groups, Math. Res. Lett. 4 (1997), no. 5, 705-717.

[51] B. Farb, N. Ivanov, The Torelli geometry and its applications: research announcement, Math. Res. Lett. 12 (2005), no. 2-3, 293-301.

[52] B. Farb, C. Leininger, D. Margalit, The lower central series and pseudoAnosov dilatations. Amer. J. Math. 130 (2008), no. 3, 799-827.

[53] B. Farb, H. Masur, Teichmüller geometry of moduli space. II. $\mathcal{M}(S)$ seen from far away, In the tradition of Ahlfors-Bers, V, 71-79, Contemp. Math., 510, Amer. Math. Soc., Providence, RI, 2010.

[54] B. Farb, R. Schwartz, The large-scale geometry of Hilbert modular groups. J. Differential Geom. 44 (1996), no. 3, 435-478.

[55] D. Gaboriau, F. Paulin, Sur les immeubles hyperboliques, Geom. Dedicata 88 (2001), no. 1-3, 153-197.

[56] V. Gadre, C. Tsai, Minimal pseudo-Anosov translation lengths on the complex of curves, arXiv:1101.2692.

[57] P. Gérardin, Harmonic functions on buildings of reductive split groups. in Operator algebras and group representations, Vol. I (Neptun, 1980), 208-221, Monogr. Stud. Math., 17, Pitman, Boston, MA, 1984.

[58] M. Gromov, Volume and bounded cohomology, Inst. Hautes Études Sci. Publ. Math. No. 56 (1982), 5-99 (1983).

[59] M. Gromov, P. Pansu, Rigidity of lattices: an introduction, in Geometric topology: recent developments (Montecatini Terme, 1990), 39-137, Lecture Notes in Math., 1504, Springer, Berlin, 1991.

[60] Y. Guivarc'h, L. Ji, J. C. Taylor, Compactifications of symmetric spaces, Progress in Mathematics, 156. Birkhäuser Boston, Inc., Boston, MA, 1998. 
xiv +284 pp.

[61] Y. Guivarc'h, B. Rémy, Group-theoretic compactification of Bruhat-Tits buildings, Ann. Sci. École Norm. Sup. 39 (2006), 871-920.

[62] U. Hamenstädt, Geometry of the mapping class groups. I. Boundary amenability, Invent. Math. 175 (2009), no. 3, 545-609.

[63] U. Hamenstädt, Geometry of the complex of curves and of Teichmüller space, in Handbook of Teichmüller theory, Vol. I, 447-467, IRMA Lect. Math. Theor. Phys., 11, Eur. Math. Soc., Zürich, 2007.

[64] U. Hamenstädt, Train tracks and the Gromov boundary of the complex of curves, in Spaces of Kleinian groups, 187-207, London Math. Soc. Lecture Note Ser., 329, Cambridge Univ. Press, Cambridge, 2006.

[65] U. Hamenstädt, Geometry of the mapping class groups III: Quasi-isometric rigidity, arXiv:math/0512429v2.

[66] J. Harer, The cohomology of the moduli space of curves, in Theory of moduli (Montecatini Terme, 1985), pp. 138-221, Lecture Notes in Math., 1337, Springer, Berlin, 1988.

[67] J. Harer, The virtual cohomological dimension of the mapping class group of an orientable surface, Invent. Math. 84 (1986), no. 1, 157-176.

[68] K. Hartshorn, Heegaard splittings of Haken manifolds have bounded distance, Pacific J. Math. 204 (2002), no. 1, 61-75.

[69] W. Harvey, Geometric structure of surface mapping class groups, in Homological group theory (Proc. Sympos., Durham, 1977), pp. 255-269, London Math. Soc. Lecture Note Ser., 36, Cambridge Univ. Press, Cambridge-New York, 1979.

[70] W. Harvey, Boundary structure of the modular group, in Riemann surfaces and related topics: Proceedings of the 1978 Stony Brook Conference (State Univ. New York, Stony Brook, N.Y., 1978), pp. 245-251, Ann. of Math. Stud., 97, Princeton Univ. Press, Princeton, N.J., 1981.

[71] W. Harvey, Remarks on the curve complex: classification of surface homeomorphisms, in Kleinian groups and hyperbolic 3-manifolds (Warwick, 2001), 165-179, London Math. Soc. Lecture Note Ser., 299, Cambridge Univ. Press, Cambridge, 2003.

[72] W. Harvey, Modular groups-geometry and physics. in Discrete groups and geometry (Birmingham, 1991), 94-103, London Math. Soc. Lecture Note Ser., 173, Cambridge Univ. Press, Cambridge, 1992.

[73] W. Harvey, Modular groups and representation spaces. in Geometry of group representations (Boulder, CO, 1987), 205-214, Contemp. Math., 74, Amer. Math. Soc., Providence, RI, 1988.

[74] A. Hatcher, W. Thurston, A presentation for the mapping class group of a closed orientable surface, Topology 19 (1980), no. 3, 221-237. 
[75] A. Hatcher, K. Vogtmann, The complex of free factors of a free group. Quart. J. Math. Oxford Ser. (2) 49 (1998), no. 196, 459-468.

[76] T. Hattori, Asymptotic geometry of arithmetic quotients of symmetric spaces, Math. Z. 222 (1996), 247-277

[77] J. Hempel, 3-manifolds as viewed from the curve complex, Topology 40 (2001), no. 3, 631-657.

[78] S. Hirose, A complex of curves and a presentation for the mapping class group of a surface, Osaka J. Math. 39 (2002), no. 4, 795-820.

[79] E. Irmak, Superinjective simplicial maps of complexes of curves and injective homomorphisms of subgroups of mapping class groups, Topology 43 (2004), no. $3,513-541$.

[80] N. Ivanov, Mapping class groups, in Handbook of geometric topology, pp. 523-633, North-Holland, Amsterdam, 2002.

[81] N. Ivanov, Automorphism of complexes of curves and of Teichmüller spaces, Internat. Math. Res. Notices 1997, no. 14, 651-666.

[82] N. Ivanov, Isometries of Teichmüller spaces from the point of view of Mostow rigidity, in Topology, ergodic theory, real algebraic geometry, 131-149, Amer. Math. Soc. Transl. Ser. 2, 202, Amer. Math. Soc., Providence, RI, 2001.

[83] N. Ivanov, Attaching corners to Teichmüller space, Leningrad Math. J. 1 (1990), no. 5, 1177-1205

[84] N. Ivanov, Action of Möbius transformations on homeomorphisms: stability and rigidity, Geom. Funct. Anal. 6 (1996), no. 1, 79-119.

[85] N. Ivanov, Subgroups of Teichmüller modular groups. Translations of Mathematical Monographs, 115. American Mathematical Society, Providence, RI, 1992. xii+127 pp.

[86] N. Ivanov, L. Ji, Infinite topology of curve complexes and non-Poincaré duality of Teichmüller modular groups, Enseign. Math. (2) 54 (2008), no. 3-4, 381-395.

[87] L. Ji, Buildings and their applications in geometry and topology, Asian J. Math. 10 (2006), no. 1, 11-80.

[88] L. Ji, From symmetric spaces to buildings, curve complexes and outer spaces, Innov. Incidence Geom. 10 (2009), 33-80.

[89] L. Ji, Integral Novikov conjectures and arithmetic groups containing torsion elements, Comm. Anal. Geom. 15 (2007), no. 3, 509-533.

[90] L. Ji, Arithmetic groups, mapping class groups, related groups, and their associated spaces, in Proceedings of the Fourth International Congress of Mathematicians, AMS/IP Stud. Adv. Math., vol. 48, pp. 127-175, Amer. Math. Soc., Providence, RI, 2010.

[91] L. Ji, Simplicial volume of moduli spaces of Riemann surfaces, preprint 2011. 
[92] L. Ji, Large scale geometry, compactifications and the integral Novikov conjectures for arithmetic groups. in Third International Congress of Chinese Mathematicians. Part 1, 2, 317-344, AMS/IP Stud. Adv. Math., 42, pt. 1, 2, Amer. Math. Soc., Providence, RI, 2008.

[93] L. Ji, The integral Novikov conjectures for S-arithmetic groups. I. K-Theory 38 (2007), no. 1, 35-47.

[94] L. Ji, R. MacPherson, Geometry of compactifications of locally symmetric spaces, Ann. Inst. Fourier (Grenoble) 52 (2002), no. 2, 457-559.

[95] L. Ji, S. Wolpert, A cofinite universal space for proper actions for mapping class groups, In the tradition of Ahlfors-Bers. V, 151-163, Contemp. Math., 510, Amer. Math. Soc., Providence, RI, 2010.

[96] J. Johnson, Heegaard splittings and the pants complex, Algebr. Geom. Topol. 6 (2006), 853-874.

[97] J. Johnson, Bridge Number and the Curve Complex, arXiv:math/0603102.

[98] I. Kapovich, N. Benakli, Boundaries of hyperbolic groups. in Combinatorial and geometric group theory, 39-93, Contemp. Math., 296, Amer. Math. Soc., Providence, RI.

[99] I. Kapovich, M. Lustig, Geometric intersection number and analogues of the curve complex for free groups. Geom. Topol. 13 (2009), no. 3, 1805-1833.

[100] Y. Kida, The mapping class group from the viewpoint of measure equivalence theory, Mem. Amer. Math. Soc. 196 (2008), no. 916, viii+190 pp.

[101] Y. Kida, Automorphisms of the Torelli complex and the complex of separating curves, arXiv:0909.4718.

[102] Y. Kim, The Thurston boundary of Teichmüller space and complex of curves, Topology Appl. 154 (2007), no. 3, 675-682.

[103] E. Klarreich, The boundary at infinity of the curve complex and the relative Teichmüller space, preprint, http://www.msri.org/people/members/klarreic/curvecomplex.ps.

[104] B. Kleiner, B. Leeb, Rigidity of quasi-isometries for symmetric spaces and Euclidean buildings, Inst. Hautes Études Sci. Publ. Math. No. 86 (1997), 115-197.

[105] M. Korkmaz, Automorphisms of complexes of curves on punctured spheres and on punctured tori, Topology Appl. 95 (1999), no. 2, 85-111.

[106] M. Korkmaz, A. Papadopoulos, On the arc and curve complex of a surface, Math. Proc. Cambridge Philos. Soc. 148 (2010), no. 3, 473-483.

[107] J. Lafont, B. Schmidt, Simplicial volume of closed locally symmetric spaces of non-compact type, Acta Math. 197 (2006), no. 1, 129-143.

[108] E. Landvogt, A compactification of the Bruhat-Tits building, Lecture Notes in Math. 1619, Springer, 1996, viii+152 pp.

[109] B. Leeb, A characterization of irreducible symmetric spaces and Euclidean buildings of higher rank by their asymptotic geometry, Bonner Mathematis- 
che Schriften, 326. Universität Bonn, Mathematisches Institut, Bonn, 2000. $\mathrm{ii}+42 \mathrm{pp}$.

[110] E. Leuzinger, Tits geometry, arithmetic groups, and the proof of a conjecture of Siegel, J. Lie Theory 14 (2004), no. 2, 317-338.

[111] E. Leuzinger, Reduction theory for mapping class groups and applications to moduli spaces, arXiv:0801.1589.

[112] T. Li, Saddle tangencies and the distance of Heegaard splittings, Algebr. Geom. Topol. 7 (2007), 1119-1134.

[113] C. Löh, R. Sauer, Simplicial volume of Hilbert modular varieties, Comment. Math. Helv. 84 (2009), no. 3, 457-470.

[114] C. Löh, R. Sauer, Degree theorems and Lipschitz simplicial volume for nonpositively curved manifolds of finite volume, J. Topol. 2 (2009), no. 1, $193-225$.

[115] E. Looijenga, Connectivity of complexes of separating curves, arXiv:1001.0823.

[116] W. Lück, Survey on classifying spaces for families of subgroups, in Infinite groups: geometric, combinatorial and dynamical aspects, 269-322, Progr. Math., 248, Birkhäuser, Basel, 2005.

[117] F. Luo, Automorphisms of the complex of curves, Topology 39 (2000), no. 2, 283-298.

[118] J. Maher, Linear progress in the complex of curves, Trans. Amer. Math. Soc. 362 (2010), no. 6, 2963-2991.

[119] A. Marden, The geometry of finitely generated kleinian groups, Ann. of Math. (2) 99 (1974), 383-462.

[120] A. Marden, Deformations of Kleinian groups, in Handbook of Teichmüller theory. Vol. I, 411-446, IRMA Lect. Math. Theor. Phys., 11, Eur. Math. Soc., Zürich, 2007.

[121] A. Marden, Outer circles. An introduction to hyperbolic 3-manifolds, Cambridge University Press, Cambridge, 2007. xviii+427 pp.

[122] D. Margalit, Automorphisms of the pants complex, Duke Math. J. 121 (2004), no. 3, 457-479.

[123] G. Margulis, Non-uniform lattices in semisimple algebraic groups, in Lie groups and their representations (Proc. Summer School on Group Representations of the Bolyai János Math. Soc., Budapest, 1971), pp. 371-553. Halsted, New York, 1975.

[124] G. Margulis, Discrete subgroups of semisimple Lie groups, Ergebnisse der Mathematik und ihrer Grenzgebiete (3), 17. Springer-Verlag, Berlin, 1991. $\mathrm{x}+388 \mathrm{pp}$.

[125] H. Masur, Y. Minsky, Quasiconvexity in the curve complex, In the tradition of Ahlfors and Bers, III, pp. 309-320, Contemp. Math., 355, Amer. Math. Soc., Providence, RI, 2004. 
[126] H. Masur, Y. Minsky, Geometry of the complex of curves. II. Hierarchical structure, Geom. Funct. Anal. 10 (2000), no. 4, 902-974.

[127] H. Masur, Y. Minsky, Geometry of the complex of curves. I. Hyperbolicity, Invent. Math. 138 (1999), no. 1, 103-149.

[128] H. Masur, M. Wolf, The Weil-Petersson isometry group, Geom. Dedicata 93 (2002), 177-190.

[129] J. D. McCarthy, A. Papadopoulos, Simplicial actions of mapping class groups, Handbook of Teichmüller theory, vol III, ??-??, IRMA Lect. Math. Theor. Phys., Eur. Math. Soc., Zürich, 2011.

[130] Y. Minsky, The classification of Kleinian surface groups. I. Models and bounds, Ann. of Math. (2) 171 (2010), no. 1, 1-107.

[131] Y. Minsky, Curve complexes, surfaces and 3-manifolds, in International Congress of Mathematicians. Vol. II, pp. 1001-1033, Eur. Math. Soc., Zürich, 2006.

[132] Y. Minsky, End invariants and the classification of hyperbolic 3-manifolds, on Current developments in mathematics, 2002, 181-217, Int. Press, Somerville, MA, 2003.

[133] Y. Minsky, Combinatorial and geometrical aspects of hyperbolic 3manifolds, in Kleinian groups and hyperbolic 3-manifolds, 3-40, London Math. Soc. Lecture Note Ser., 299, Cambridge Univ. Press, Cambridge, 2003.

[134] Y. Minsky, Curve complexes, surfaces and 3-manifolds, in International Congress of Mathematicians, Vol. II, 1001-1033, Eur. Math. Soc., Zürich, 2006.

[135] M. Mj, Cannon-Thurston maps for pared manifolds of bounded geometry. Geom. Topol. 13 (2009), no. 1, 189-245.

[136] M. Mj, Mapping class groups and interpolating complexes: rank. J. Ramanujan Math. Soc. 24 (2009), no. 4, 341-357.

[137] M. Moore, M. Rathbun, High distance knots in closed 3-manifolds, arXiv:0911.3675.

[138] G. Mostow, Strong rigidity of locally symmetric spaces, Annals of Mathematics Studies, No. 78. Princeton University Press, Princeton, N.J.; University of Tokyo Press, Tokyo, 1973. v+195 pp.

[139] A. Moy, Displacement functions on the Bruhat-Tits building, In The mathematical legacy of Harish-Chandra, 483-499, Proc. Sympos. Pure Math., 68, Amer. Math. Soc., Providence, RI, 2000.

[140] P. Nowak, G. Yu, What is ... property A?, Notices Amer. Math. Soc. 55 (2008), no. 4, 474-475.

[141] L. Paris, Actions and irreducible representations of the mapping class group, Math. Ann. 322 (2002), no. 2, 301-315. 
[142] D. Patterson, The Teichmïler spaces are distinct, Proc. Amer. Math. Soc. 35 (1972), 179-182.

[143] R. Penner, J. Harer, Combinatorics of train tracks. Annals of Mathematics Studies, 125. Princeton University Press, Princeton, NJ, 1992. xii+216 pp.

[144] G. Prasad, Strong rigidity of $\mathbb{Q}$-rank 1 lattices, Invent. Math. 21 (1973), $255-286$.

[145] A. Putman, A note on the connectivity of certain complexes associated to surfaces, Enseign. Math. (2) 54 (2008), no. 3-4, 287-301.

[146] K. Rafi, A combinatorial model for the Teichmüller metric, Geom. Funct. Anal. 17 (2007), no. 3, 936-959.

[147] K. Rafi, S. Schleimer, Covers and the curve complex, Geom. Topol. 13 (2009), no. 4, 2141-2162.

[148] K. Rafi, S. Schleimer, Curve complexes are rigid, arXiv:0710.3794.

[149] J. Ratcliffe, Foundations of hyperbolic manifolds, Second edition. Graduate Texts in Mathematics, 149. Springer, New York, 2006. xii+779 pp.

[150] B. Rémy, A. Thuillier, A. Werner, Bruhat-Tits theory from Berkovich's point of view. I. Realizations and compactifications of buildings. Ann. Sci. Éc. Norm. Supér. (4) 43 (2010), no. 3, 461-554.

[151] B. Rémy, A. Thuillier, A. Werner, Bruhat-Tits theory from Berkovich's point of view. II. Satake compactifications, arXiv:0907.3264.

[152] M. Ronan, From Galois and Lie to Tits buildings,, in The Coxeter legacy, 45-62, Amer. Math. Soc., Providence, RI, 2006,

[153] M. Ronan, J. Tits, Building buildings, Math. Ann. 278 (1987), no. 1-4, 291-306.

[154] H.L. Royden, Automorphisms and isometries of Teichmüller space, in $A d-$ vances in the Theory of Riemann Surfaces (Proc. Conf., Stony Brook, N.Y., 1969), pp. 369-383, Ann. of Math. Studies, No. 66., Princeton Univ. Press.

[155] T. Saito, Genus one 1-bridge knots as viewed from the curve complex, Osaka J. Math. 41 (2004), no. 2, 427-454.

[156] T. Saito, R. Yamamoto, Complexity of open book decompositions via arc complex, J. Knot Theory Ramifications 19 (2010), no. 1, 55-69

[157] M. Scharlemann, Proximity in the curve complex: boundary reduction and bicompressible surfaces, Pacific J. Math. 228 (2006), no. 2, 325-348.

[158] M. Scharlemann, M. Tomova, Alternate Heegaard genus bounds distance, Geom. Topol. 10 (2006), 593-617

[159] P. Scott, C. T. Wall, Topological methods in group theory. in Homological group theory (Proc. Sympos., Durham, 1977), pp. 137-203, London Math. Soc. Lecture Note Ser., 36, Cambridge Univ. Press, Cambridge-New York, 1979. 
[160] R. Schwartz, The quasi-isometry classification of rank one lattices, Inst. Hautes Études Sci. Publ. Math., 82 (1995), 133-168.

[161] R. Schwartz, Quasi-isometric rigidity and Diophantine approximation. Acta Math. 177 (1996), no. 1, 75-112.

[162] K. Shackleton, Combinatorial rigidity in curve complexes and mapping class groups, Pacific J. Math. 230 (2007), no. 1, 217-232.

[163] B. Szepietowski, A presentation for the mapping class group of a nonorientable surface from the action on the complex of curves, Osaka J. Math. 45 (2008), no. 2, 283-326.

[164] W. Thurston, On the geometry and dynamics of diffeomorphisms of surfaces, Bull. Amer. Math. Soc. (N.S.) 19 (1988), no. 2, 417-431.

[165] P. Tukia, A rigidity theorem for Möbius groups, Invent. Math. 97 (1989), no. $2,405-431$.

[166] J. Tits, Buildings of spherical type and finite BN-pairs, Lecture Notes in Mathematics, Vol. 386. Springer-Verlag, Berlin-New York, 1974. x+299 pp.

[167] J. Tits, On buildings and their applications, Proceedings of the International Congress of Mathematicians (Vancouver, B. C., 1974), Vol. 1, pp. 209-220. Canad. Math. Congress, Montreal, Que., 1975.

[168] M. Tomova, Distance of Heegaard splittings of knot complements, Pacific J. Math. 236 (2008), no. 1, 119-138.

[169] B. Wajnryb, A simple presentation for the mapping class group of an orientable surface. Israel J. Math. 45 (1983), no. 2-3, 157-174.

[170] A. Werner, Non-Archimedean intersection indices on projective spaces and the Bruhat-Tits building for PGL, Ann. Inst. Fourier (Grenoble) 51 (2001), no. $6,1483-1505$.

[171] A. Werner, Compactifications of Bruhat-Tits buildings associated to linear representations. Proc. Lond. Math. Soc. (3) 95 (2007), no. 2, 497-518.

[172] A. Werner, Compactification of the Bruhat-Tits building of PGL by lattices of smaller rank, Doc. Math. 6 (2001) 315-341.

[173] A. Werner, Compactification of the Bruhat-Tits building of PGL by seminorms, Math. Z. 248 (2004) 511-526.

[174] S. Wolpert, Geometry of the Weil-Petersson completion of Teichmüller space, in Surveys in differential geometry, Vol. VIII (Boston, MA, 2002), 357-393, Surv. Differ. Geom., VIII, Int. Press, Somerville, MA, 2003. 